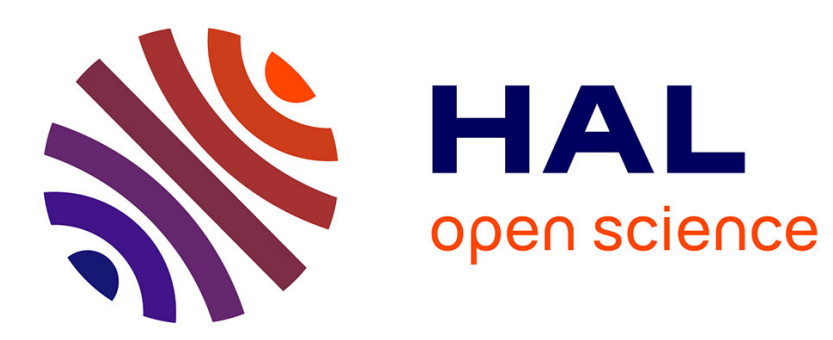

\title{
Quand est-ce que des bornes de Hardy permettent de calculer une constante de Poincaré exacte sur la droite? Laurent Miclo
}

\section{To cite this version:}

Laurent Miclo. Quand est-ce que des bornes de Hardy permettent de calculer une constante de Poincaré exacte sur la droite?. Annales de la Faculté des Sciences de Toulouse. Mathématiques., 2008, 17 (1), pp.121-192. hal-00017875

\section{HAL Id: hal-00017875 https://hal.science/hal-00017875}

Submitted on 25 Jan 2006

HAL is a multi-disciplinary open access archive for the deposit and dissemination of scientific research documents, whether they are published or not. The documents may come from teaching and research institutions in France or abroad, or from public or private research centers.
L'archive ouverte pluridisciplinaire HAL, est destinée au dépôt et à la diffusion de documents scientifiques de niveau recherche, publiés ou non, émanant des établissements d'enseignement et de recherche français ou étrangers, des laboratoires publics ou privés. 


\title{
Quand est-ce que des bornes de Hardy permettent de calculer une constante de Poincaré exacte sur la droite?
}

\author{
Laurent Miclo \\ Laboratoire d'Analyse, Topologie, Probabilités \\ U.P. et C.N.R.S. \\ Marseille, France
}

\begin{abstract}
Résumé
Classiquement, des inégalités de Hardy permettent d'estimer le trou spectral d'une diffusion réelle à un facteur 4 près. L'objectif de ce papier est d'essayer de mieux appréhender cette constante fluctuante, du moins dans un contexte symétrique. Notamment on donnera un critère asymptotique simple assurant qu'elle vaut exactement 4 . L'argument sous-jacent consiste à voir le trou spectral comme une fonctionnelle semi-explicite et surtout monotone en un réarrangement des données du problème. Pour l'exhiber, on aura recours à des propriétés de régularité de la constante de Poincaré correspondante et on fera certains liens avec les méthodes de chemins, les premières valeurs propres de Dirichlet, les équations de Sturm-Liouville et les fonctionnelles browniennes, la plupart ayant déjà été observés par divers auteurs. Enfin on étendra les résultats obtenus au cas des processus de vie et de mort sur $\mathbb{Z}$, mais toujours dans un cadre symétrique. Notre espoir est que cette démarche pourra s'adapter pour permettre d'appliquer finement les constantes de Hardy à des inégalités fonctionnelles plus ardues que celle de Poincaré.
\end{abstract}

Mots clés : diffusion réelle, trou spectral, inégalité de Poincaré, inégalité de Hardy, méthode des chemins, première valeur propre de Dirichlet, équation de Sturm-Liouville, théorème de RayKnight, transformées de Laplace de fonctionnelles browniennes quadratiques, processus de vie et de mort.

MSC2000 : premièrement : 60J60, secondairement : 49R50, 46E35, 34B24, 60J65, 60J27. 


\section{Introduction}

Dans ces notes nous allons nous intéresser à des évaluations précises de constantes de Poincaré sur $\mathbb{R}($ ou sur $\mathbb{Z})$ en appliquant directement des inégalités de Hardy à des méthodes de chemins et en ayant recours à certaines estimations asymptotiques.

Plus précisément, soit $\mu$ une probabilité sur $\mathbb{R}$ (muni de sa tribu borélienne $\mathcal{R}$ ) et $\nu$ une mesure $\operatorname{sur}(\mathbb{R}, \mathcal{R})$, non nécessairement de masse totale finie, mais dans un premier temps supposée régulière et $\sigma$-finie (on indiquera dans la section suivante comment se débarrasser de ces deux hypothèses). La constante de Poincaré $A(\mu, \nu) \in \overline{\mathbb{R}}_{+}$associée à un tel couple est l'infimum de l'ensemble des réels $A \in \mathbb{R}_{+}$tels que

$$
\forall f \in \mathcal{C}^{1}(\mathbb{R}), \quad \operatorname{Var}(f, \mu) \leq A \nu\left[\left(f^{\prime}\right)^{2}\right]
$$

où rappelons que le terme de gauche désigne la variance de $f$ par rapport à $\mu$ qui est définie par

$$
\operatorname{Var}(f, \mu):=\frac{1}{2} \int(f(y)-f(x))^{2} \mu(d x) \mu(d y) \leq+\infty
$$

expression qui vaut aussi $\mu\left[(f-\mu[f])^{2}\right]=\mu\left[f^{2}\right]-(\mu[f])^{2}$ si $f \in \mathbb{L}^{2}(\mu)$. Cependant la formulation précédente a l'avantage d'être toujours bien définie (dans $\overline{\mathbb{R}}_{+}$) et de pouvoir s'étendre telle quelle à des mesures $\mu$ générales.

Comme cela est de tradition, la convention $\inf (\emptyset)=+\infty$ a été implicitement utilisée ci-dessus et $A(\mu, \nu)=+\infty$ équivaut à l'inexistence d'une constante $A \in \mathbb{R}_{+}$telle que (1) soit satisfait. Dans le cas contraire où $A(\mu, \nu)<+\infty$, l'inégalité (1) reste évidemment satisfaite si l'on y prend $A=A(\mu, \nu)($ si $A(\mu, \nu)=0$, on aura remarqué que la régularité et la $\sigma$-finitude de $\nu$ impliquent que $\mu$ est une masse de Dirac, voir aussi la section suivante pour ce genre de discussion) et on parle alors d'inégalité de Poincaré.

Ces dernières années, Bobkov et Götze $[3,4]$ ont donné une évaluation de $A(\mu, \nu)$ valable à un facteur 8 près et relativement explicite en termes des deux données du problème $\mu$ et $\nu$ : soit $m$ une médiane de $\mu$ (c'est-à-dire un nombre réel $m$ satisfaisant $\mu(]-\infty, m]) \geq 1 / 2$ et $\mu([m,+\infty[) \geq 1 / 2)$ et abusons un peu des notations en désignant aussi par $\nu$ la dérivée de Radon-Nikodym $d \nu / d \lambda$ (à comprendre comme un élément de $\mathbb{L}^{0}(\lambda)$ ) de $\nu$ (i.e. de sa composante absolument continue) par rapport à la mesure de Lebesgue $\lambda$. On définit ensuite

$$
\begin{aligned}
B_{+}(\mu, \nu, m) & :=\sup _{x>m} \int_{m}^{x} \frac{1}{\nu(y)} \lambda(d y) \mu([x,+\infty[) \\
B_{-}(\mu, \nu, m) & \left.\left.:=\sup _{x<m} \int_{x}^{m} \frac{1}{\nu(y)} \lambda(d y) \mu(]-\infty, x\right]\right) \\
B(\mu, \nu, m) & :=\max \left(B_{+}(\mu, \nu, m), B_{-}(\mu, \nu, m)\right)
\end{aligned}
$$

puis soient $B_{*}(\mu, \nu)$ et $B^{*}(\mu, \nu)$ respectivement l'infimum et le supremum des $B(\mu, \nu, m)$ quand $m$ parcourt l'ensemble (convexe, borné et non-vide) des médianes de $\mu$ possibles. En utilisant des inégalités de Hardy, Bobkov et Götze [3, 4] ont obtenu le résultat suivant.

Théorème 1 On a toujours

$$
\frac{1}{2} B^{*}(\mu, \nu) \leq A(\mu, \nu) \leq 4 B_{*}(\mu, \nu)
$$

ces inégalités devant être comprises dans $\overline{\mathbb{R}}_{+}$.

Profitons-en pour rappeler ce que l'on entendra ici par inégalité de Hardy. Il s'agit d'inégalités similaires à celles de Poincaré, sauf que l'on cherche à trouver la meilleure constante $C(\mu, \nu) \in \overline{\mathbb{R}}_{+}$ satisfaisant

$$
\mu\left[f^{2}\right] \leq C(\mu, \nu) \nu\left[\left(f^{\prime}\right)^{2}\right]
$$


pour toute fonction $f \in \mathcal{C}^{1}\left(\mathbb{R}_{+}\right)$vérifiant $f(0)=0$. Pour cette question, $\mu$ et $\nu$ sont simplement deux mesures sur $\mathbb{R}_{+}, \nu$ étant toujours régulière et $\sigma$-finie.

Notre principal objectif est de discuter des bornes données dans le théorème 1. Pour simplifier, on se placera dans un contexte symétrique où $\mu$ et $\nu$ vérifient de plus

$$
\forall A \in \mathcal{R}, \quad\left\{\begin{array}{l}
\mu(-A)=\mu(A) \\
\nu(-A)=\nu(A)
\end{array}\right.
$$

Il semble alors naturel de plutôt chercher à comparer $A(\mu, \nu)$ avec $B(\mu, \nu):=B(\mu, \nu, 0)$. Notamment on montrera par une preuve alternative faisant intervenir certaines fonctionnelles du mouvement brownien standard que

$$
B(\mu, \nu) \leq A(\mu, \nu) \leq 4 B(\mu, \nu)
$$

(ceci resserre un peu l'étau du théorème 1 si $\mu$ n'admet qu'une seule médiane, par exemple si le support de $\mu$ est un intervalle) et on vérifiera que l'on ne peut pas mieux faire d'une manière générale.

Puis surtout nous nous intéresserons au cas d'égalité $A(\mu, \nu)=4 B(\mu, \nu)$ en proposant le critère asymptotique suivant :

Proposition 2 Soient $b_{\mu, \nu}$ la fonction

$$
\mathbb{R}_{+} \ni x \mapsto \int_{0}^{x} \frac{1}{\nu(y)} \lambda(d y) \mu\left(\left[x,+\infty[) \in \overline{\mathbb{R}}_{+}\right.\right.
$$

et $M_{\mu} \in \overline{\mathbb{R}}_{+}$le supremum du support de $\mu$, c'est-à-dire le plus petit $M \in \overline{\mathbb{R}}_{+}$tel que $\mu(] M,+\infty[)=0$. Supposons que $\mu\left(\left\{M_{\mu}\right\}\right)=0$ et que

$$
\lim _{x \rightarrow M_{\mu}-} b_{\mu, \nu}(x)=B(\mu, \nu)
$$

(rappelons que par définition, $B(\mu, \nu):=\sup _{0<x \leq M_{\mu}} b_{\mu, \nu}(x)$ ), on est alors assuré de l'égalité $A(\mu, \nu)=$ $4 B(\mu, \nu)$.

D'une manière plus anodine, on considèrera aussi le cas d'égalité $A(\mu, \nu)=B(\mu, \nu)$, qui se rencontre beaucoup plus rarement si $0<A(\mu, \nu)<+\infty$ et si $\limsup _{x \rightarrow M_{\mu}-} b_{\mu, \nu}(x)<B(\mu, \nu)$, puisqu'alors il ne correspond qu'aux seules probabilités $\mu$ de la forme $1 / 2\left(\delta_{x}+\delta_{-x}\right)$ avec $x \in \mathbb{R}_{+}^{*}$ (i.e. les lois des multiples par $x \in \mathbb{R}_{+}^{*}$ de la variable de Bernoulli symétrique usuelle, le cas trivial de la masse de Dirac en 0 étant exclu, puisque $A\left(\delta_{0}, \nu\right)=0$ ), notons que dans cette situation, $M_{\mu}=x$, $\lim _{y \rightarrow M_{\mu}-} b_{\mu, \nu}(y)=0$ et $B(\mu, \nu)=b_{\mu, \nu}(x)$.

D'autre part, on étendra la proposition précédente à un cadre discret, mais toujours en se restreignant à une situation symétrique. Ceci fournira donc une nouvelle méthode de calcul exact de trous spectraux associés à certains processus de vie et de mort sur $\mathbb{Z}$.

A la base de ces résultats se trouve une observation très simple affirmant que $A(\mu, \nu)$ est nécessairement une fonctionnelle monotone de la fonction $\left.\widetilde{b}_{\mu, \nu}:\right] 0,1\left[\rightarrow \overline{\mathbb{R}}_{+}\right.$définie comme suit : soient $\left.\left.F_{\mu}: \mathbb{R} \ni x \mapsto \mu(]-\infty, x\right]\right)$ la fonction de répartition de $\mu$ et $F_{\mu}^{-1}$ son inverse généralisé :

$$
\forall u \in] 0,1\left[, \quad F_{\mu}^{-1}(u):=\inf \left\{x \in \mathbb{R}: F_{\mu}(x)>u\right\} \in \mathbb{R}\right.
$$

On pose alors

$$
\forall u \in] 0,1\left[, \quad \widetilde{b}_{\mu, \nu}(u):=\left|\int_{0}^{F_{\mu}^{-1}(u)} \frac{1}{\nu(y)} \lambda(d y)\right|\right.
$$

(un lien avec la fonction $b_{\mu, \nu}$ de la proposition précédente est que par exemple pour $1 / 2 \leq u<1$, on a $(1-u) \widetilde{b}_{\mu, \nu}(u)=b_{\mu, \nu} \circ F_{\mu}^{-1}(u)$, sauf éventuellement si $F_{\mu}^{-1}(u)$ est l'un des atomes de $\mu$ (eux 
sont en nombre au plus dénombrable, par contre en général l'égalité précédente n'est pas satisfaite p.p. en $u \in] 0,1[$ ), d'ailleurs en y regardant de plus près, on constate que pour tout $1 / 2 \leq u<1$, $b_{\mu, \nu}\left(F_{\mu}^{-1}(u)+\right) \leq(1-u) \widetilde{b}_{\mu, \nu}(u) \leq b_{\mu, \nu}\left(F_{\mu}^{-1}(u)\right)$, ce qui permet de voir que sous la condition $\mu\left(\left\{M_{\mu}\right\}\right)=0$, l'hypothèse $(3)$ de la proposition 2 est équivalente à $\lim _{u \rightarrow 1-}(1-u) \widetilde{b}_{\mu, \nu}(u)=B(\mu, \nu)$ et c'est sous cette forme que nous l'utiliserons, voir la section 5 pour une justification de ces affirmations).

La monotonicité mentionnée ci-dessus aurait aussi pu s'obtenir en adaptant certains arguments de [4], mais nous avons préféré en donner une approche plus probabiliste. Ceci plus par goût que par désir d'originalité, car de profonds liens entre l'inégalité de Hardy classique (correspondant à $\mu(d x)=x^{-2} \lambda(d x)$ et $\nu(d x)=\lambda(d x)$ sur $\left.\mathbb{R}_{+}\right)$et certaines fonctionnelles du mouvement brownien ont déjà été mis en évidence par Yor et ses co-auteurs (voir par exemple Donati-Martin et Yor [13], Yor [42], Pecatti et Yor [31]).

Par ailleurs, même l'esprit de la proposition 2 n'est pas sans rappeler certains résultats analytiques concernant les inégalités de Hardy classiques : déjà dans l'ouvrage [18] de Hardy, Littlewood et Pólya la bonne constante est déterminée (dans un cadre continu et discret et pour des estimées $\mathbb{L}^{p}$, avec $p>1$, plutôt qu'uniquement $\mathbb{L}^{2}$ ) et le fait que l'on puisse obtenir de telles estimations précises est relié à l'absence de minimiseur par Marcus, Mizel et Pinchover dans [24] (voir aussi Matskewich et Sobolevskii [25]). Cependant même s'ils se placent dans un contexte multidimensionnel, ces auteurs s'intéressent à des mesures particulières généralisant l'inégalité linéaire classique $: \nu$ est une mesure de Lebesgue et $\mu$ admet pour densité par rapport à $\nu$ une puissance négative $(-p$ pour des estimées $\mathbb{L}^{p}$, avec $p>1$ ) de la distance euclidienne au bord du domaine. Toutefois dans notre cadre il s'agit d'être plus prudent car on ne peut pas conclure à l'égalité $A(\mu, \nu)=4 B(\mu, \nu)$ sous la seule condition que $\mu\left(\left\{M_{\mu}\right\}\right)=0$ et que la fonction $b_{\mu, \nu}$ "atteint" son supremum en l'infini. Ainsi on donnera un exemple où $\lim \sup _{x \rightarrow+\infty} b_{\mu, \nu}(x)=B(\mu, \nu)$ avec $\mu\left(\left\{M_{\mu}\right\}\right)=0$ et où pourtant la conclusion de la proposition 2 est fausse (pire, on aura même $A(\mu, \nu)=B(\mu, \nu) \in \mathbb{R}_{+}^{*}$ ).

Heuristiquement l'hypothèse de la proposition 2 exige que $\mu$ soit suffisamment lourde au voisinage gauche de $M_{\mu}$ (représentant "l'infini") par rapport à $\nu$, ou inversement si $\mu$ est fixée, que $\nu$ y soit relativement légère. Voici quelques exemples simples allant dans cette direction.

Corollaire 3 Soit $U: \mathbb{R} \rightarrow \mathbb{R}$ une fonction paire vérifiant $U(0)=\ln (2)$, divergente vers $+\infty$ en l'infini, absolument continue et dont la dérivée faible de Radon-Nikodym $U^{\prime}$ est p.p. strictement positive sur $\mathbb{R}_{+}$. Notons $\mu$ la probabilité (respectivement $\nu$ la mesure) admettant $\left|U^{\prime}\right| \exp (-U)$ (resp. $\left.\exp (-U) /\left|U^{\prime}\right|\right)$ pour densité par rapport à $\lambda$.

On a alors $A(\mu, \nu)=4$.

\section{Preuve :}

En effet, on calcule que pour tout $x \in \mathbb{R}_{+}$,

$$
\begin{aligned}
b_{\mu, \nu}(x) & =\int_{0}^{x} U^{\prime}(y) \exp (U(y)) \lambda(d y) \int_{x}^{+\infty} U^{\prime}(y) \exp (-U(y)) \lambda(d y) \\
& =[\exp (U(y))]_{0}^{x}[-\exp (-U(y))]_{x}^{+\infty} \\
& =1-2 \exp (-U(x))
\end{aligned}
$$

fonction qui croît vers 1 en $+\infty$.

Ce résultat est susceptible de nombreuses variantes, par exemple :

Corollaire 4 Soit $U: \mathbb{R} \rightarrow \mathbb{R}$ une fonction paire de classe $\mathcal{C}^{2}$, convexe, strictement croissante sur $\mathbb{R}_{+}$et vérifiant $U^{\prime \prime} \ll\left(U^{\prime}\right)^{2}$ en $+\infty$. Soit $\mu$ la probabilité admettant $\exp (-U) / Z$ pour densité par rapport à $\lambda$ avec $Z:=\int \exp (-U) d \lambda$. Considérons aussi la mesure symétrique sur $(\mathbb{R}, \mathcal{R})$ donnée par $\nu(d x)=\exp (-U(x)) /\left(U^{\prime}(x)\right)^{2} \lambda(d x)$. On a alors $A(\mu, \nu)=4 Z^{-1}$.

\section{Preuve :}


Pour tout $x>0$, on a

$$
\begin{aligned}
\int_{x}^{+\infty} \exp (-U(y)) \lambda(d y) & =\int_{x}^{+\infty} \frac{1}{U^{\prime}(y)} U^{\prime}(y) \exp (-U(y)) \lambda(d y) \\
& =\left[-\frac{\exp (-U(y))}{U^{\prime}(y)}\right]_{x}^{+\infty}-\int_{x}^{+\infty} \frac{U^{\prime \prime}(y)}{\left(U^{\prime}(y)\right)^{2}} \exp (-U(y)) \lambda(d y) \\
& \leq \frac{\exp (-U(x))}{U^{\prime}(x)}
\end{aligned}
$$

(qui est bien fini, car la convexité et la stricte croissance de $U$ entraînent que $U^{\prime}>0$ sur $\mathbb{R}_{+}^{*}$, on aura aussi remarqué que ces propriétés impliquent la finitude de $Z$ ). De plus en utilisant l'hypothèse faite sur $U^{\prime \prime}$, on obtient l'équivalent suivant pour $x$ grand

$$
\begin{aligned}
\int_{x}^{+\infty} \exp (-U(y)) \lambda(d y) & \sim \int_{x}^{+\infty}\left(1+\frac{U^{\prime \prime}(y)}{\left(U^{\prime}(y)\right)^{2}}\right) \exp (-U(y)) \lambda(d y) \\
& =\frac{\exp (-U(x))}{U^{\prime}(x)}
\end{aligned}
$$

De même on calcule que pour tout $x \geq 0$,

$$
\begin{aligned}
\int_{0}^{x}\left(U^{\prime}\right)^{2} \exp (U) d \lambda & =\left[U^{\prime} \exp (U)\right]_{0}^{x}-\int_{0}^{x} U^{\prime \prime} \exp (U) d \lambda \\
& \leq U^{\prime}(x) \exp (U(x))
\end{aligned}
$$

et que le membre de gauche et de droite sont équivalents pour $x$ grand. Le résultat annoncé découle alors directement de la proposition 2 .

Dans la proposition 2, le comportement de $\nu$ exactement en 0 est sans importance, ainsi le résultat précédent reste valable si ses hypothèses sont satisfaites uniquement sur $\mathbb{R}^{*}$ (on pourrait aussi supposer seulement que $U^{\prime}$ est absolument continu sur ]0, $+\infty$ [ et que l'une des versions de sa dérivée est négligeable devant $\left(U^{\prime}\right)^{2}$ en $+\infty$, cette dernière condition n'est pas satisfaite par exemple si $U^{\prime \prime}$ admet de forts pics et il n'est pas clair que l'on puisse s'en passer). En particulier, on peut considérer le potentiel

$$
U_{\alpha}: \mathbb{R} \ni x \mapsto|x|^{\alpha} \in \mathbb{R}_{+}
$$

avec $\alpha \geq 1$, et notons par $\mu_{\alpha}$ et $\nu_{\alpha}$ les mesures correspondantes construites dans le corollaire précédent. Dans le cas $\alpha=1$, on retrouve un résultat bien connu pour la distribution exponentielle symétrisée. Pour $\alpha>1$, la mesure $\nu_{\alpha}$ admet une densité singulière en 0 , mais ce n'est pas très grave. La théorie des formes de Dirichlet (voir par exemple le livre [15] de Fukushima, Ōshima et Takeda) permet d'associer un unique générateur markovien $L_{\alpha}$ auto-adjoint dans $\mathbb{L}^{2}\left(\mu_{\alpha}\right)$ à la fermeture de la forme quadratique $\mathcal{D}_{\alpha} \ni f \mapsto \nu_{\alpha}\left[\left(f^{\prime}\right)^{2}\right]$, avec $\mathcal{D}_{\alpha}:=\left\{f \in \mathcal{C}_{\mathrm{c}}^{1}(\mathbb{R}): \nu_{\alpha}\left[\left(f^{\prime}\right)^{2}\right]<+\infty\right\}$. Cet opérateur $L_{\alpha}$ agit notamment sur les fonctions $f$ de classe $\mathcal{C}^{2}$ constantes dans un voisinage de 0 et à support compact par

$$
\begin{aligned}
\forall x \in \mathbb{R}, \quad L_{\alpha}[f](x) & =\frac{\nu_{\alpha}}{\mu_{\alpha}}(x) f^{\prime \prime}(x)+\frac{\nu_{\alpha}^{\prime}}{\mu_{\alpha}}(x) f^{\prime}(x) \\
& =Z_{\alpha}\left[\alpha^{-2}|x|^{-2(\alpha-1)} f^{\prime \prime}(x)-\operatorname{sign}(x)\left(\alpha^{-1}|x|^{1-\alpha}+2 \alpha^{-2}(\alpha-1)|x|^{1-2 \alpha}\right) f^{\prime}(x)\right]
\end{aligned}
$$

La constante $1 / A\left(\mu_{\alpha}, \nu_{\alpha}\right)$ correspond alors au trou spectral de $L_{\alpha}$ dans $\mathbb{L}^{2}\left(\mu_{\alpha}\right)$.

Dans le genre de situation considérée dans la proposition $2, A(\mu, \nu)$ mesure d'une certaine manière la difficulté que rencontre le processus sous-jacent à "revenir de l'infini" plus que les difficultés locales d'atteinte de l'équilibre $\left(\right.$ dans $\left.\mathbb{L}^{2}(\mu)\right)$. En effet, à chaque fois que, outre les hypothèses de la 
proposition 2, la fonction $b_{\mu, \nu}$ n'atteint pas son supremum sur $\left[0, M_{\mu}[\right.$, on peut de manière locale légèrement y modifier la mesure $\nu$ sans altérer ni $B(\mu, \nu)$ ni la condition de la proposition 2 , et par conséquent sans changer $A(\mu, \nu)$ (car on aura remarqué qu'alors $\int_{0}^{1} 1 / \nu d \lambda=+\infty$ ). On aurait pu de la sorte gommer la vilaine singularité de $\nu_{\alpha}$ en 0 sans pour autant perturber la valeur de $A\left(\mu_{\alpha}, \nu_{\alpha}\right)$.

Par contre, on aura noté que pour $\alpha>1$, la fonction $b_{\mu_{\alpha}, \mu_{\alpha}}$ atteint son maximum en un unique point $x_{\alpha}$ de $] 0,+\infty\left[\right.$. Dans ce cas l'opérateur sous-jacent $f \mapsto\left(f^{\prime \prime}(x)-\alpha x^{\alpha-1} f^{\prime}(x)\right)_{x \in \mathbb{R}}$ fait revenir très rapidement de l'infini la diffusion qu'il génère; ce n'est pas cet effort qui contribue de manière prépondérante dans le trou spectral $1 / A\left(\mu_{\alpha}, \mu_{\alpha}\right)$, mais des difficultés locales de convergence à l'équilibre (toujours dans un sens $\mathbb{L}^{2}(\mu)$ ). A cet égard, on peut se demander s'il n'existerait pas des points "plus retardants" que les autres, en conjecturant que les pires sont justement $x_{\alpha}$ et $-x_{\alpha}$. Mais ceci n'est pas évident, car quand la condition de la proposition 2 n'est pas remplie, tous les points (de $]-M_{\mu}, M_{\mu}[$ ) contribuent un peu à $A(\mu, \nu)$, au sens où de petites diminutions de $\nu$ au voisinage de ces points (c'est-à-dire des dopages correspondants du générateur, à probabilité réversible $\mu$ fixée), entraînent une augmentation stricte du trou spectral $1 / A(\mu, \nu)$. Cela est possible puisque dans cette situation les liens entre $A(\mu, \nu)$ et $B(\mu, \nu)$ sont distendus (i.e. valables à un facteur près), et n'est pas non plus sans relation avec l'existence de vecteurs propres associés au trou spectral (ou de manière équivalente, avec celle de minimiseurs pour $A(\mu, \nu)$ ). Ce sujet et notamment des propriétés quantitatives de monotonicité de $A(\mu, \nu)$ en tant que fonction de $\widetilde{b}_{\mu, \nu}$, mériteraient éventuellement d'être approfondis.

Le plan du papier est le suivant. Dans la prochaine section on réduira l'évaluation de $A(\mu, \nu)$ à un problème de Sturm-Liouville sur ] - 1, 1[ avec conditions de Dirichlet au bord. On réécrira ce dernier en terme de finitude d'une espérance d'exponentielle de fonctionnelle quadratique du mouvement brownien standard dans la section 3, par le biais d'un théorème de Ray-Knight. Ceci nous permettra dans la section 4 d'obtenir le résultat de comparaison auquel nous faisions allusion ci-dessus (au travers des fonctions $\widetilde{b}_{\mu, \nu}$ ). On s'en servira pour prouver la proposition 2 dans la section 5 . Dans la suivante on démontrera deux petites assertions faites précédemment, l'une concernant les cas d'égalité $A(\mu, \nu)=B(\mu, \nu)$ et l'autre l'insuffisance de la condition $\lim \sup _{x \rightarrow+\infty} b_{\mu, \nu}(x)=B(\mu, \nu)$ pour l'identité $A(\mu, \nu)=4 B(\mu, \nu)$. Enfin dans une dernière section on traitera le cas discret.

Pour terminer cette introduction, signalons deux extensions que nous aimerions aborder dans d'éventuelles futures études et qui ont servi de motivations pour ce papier préliminaire "dans le cas facile de l'inégalité de Poincaré" (c'est par ce désir de faire une mise au point que nous n'avons pas hésité à discuter de questions pourtant très classiques, parfois de manière trop soignée au risque d'ennuyer le lecteur).

- Tout d'abord il s'agirait de remplacer dans (1) la variance par une entropie. Des bornes de type Hardy pour la constante correspondante de Sobolev logarithmique ont déjà été présentées par Bobkov et Götze [3] puis améliorées par Barthe et Roberto [2]. Cependant il n'est peut-être pas déraisonnable de penser qu'une constante exacte pourrait être obtenue sous des conditions asymptotiques appropriées, similaires au critère de la proposition 2 (éventuellement on pourrait essayer de comprendre quel est le type d'entropie généralisée (voir celles considérées dans les articles de Chafaï [5] ou de Barthe, Cathiaux et Roberto [1]) pour lesquelles cette possibilité existe). Une application serait de retrouver facilement l'équivalent de la constante de Sobolev logarithmique associée à des opérateurs de Laguerre de petit paramètre (obtenue laborieusement dans [28]), voire de la calculer. Il s'agit là d'une situation non-symétrique, mais tout suggère que cette constante est dictée par des comportements en "l'infini à gauche", qui en l'occurrence est $0_{+}$.

- Surtout, on voudrait modifier le terme d'énergie de (1) en le remplaçant par $\int H\left(\left(f^{\prime} / f\right)^{2}\right) f^{2} d \nu$, où $H: \mathbb{R}_{+} \rightarrow \mathbb{R}_{+}$est une fonction convexe vérifiant $H(0)=0$ et $H^{\prime}(0)>0$. Plus précisément on voudrait évaluer la meilleure constante $A>0$ telle que

$$
\forall f \in \mathcal{C}^{1}(\mathbb{R}), \quad \operatorname{Ent}\left(f^{2}, \mu\right) \leq \int H\left(A\left(\frac{f^{\prime}}{f}\right)^{2}\right) f^{2} d \nu
$$


(ou même seulement avec une variance dans le membre de gauche). Pour l'intérêt de ces inégalités de Sobolev logarithmiques modifiées, on renvoie par exemple à [16].

\section{Des équations de Sturm-Liouville avec condition de Dirichlet}

Cette section est fortement influencée d'une part par les méthodes de chemins et d'autre part par les manipulations de réductions du problème effectuées par Bobkov et Götze dans [4]. Concernant les méthodes de chemins, l'auteur les a apprises dans [20] de Holley et Stroock (voir aussi Diaconis et Stroock [12]) pour un cadre discret (fini) et dans [21] de Holley, Kusuoka, et Stroock pour un cadre continu (mais compact). Pour une référence générale relative à leur application aux chaînes de Markov, on peut renvoyer au cours de Saloff-Coste [40] et plus précisément à l'article de Kahale [22] pour une discussion de leur caractère optimal, qui est vérifiée dans le cas "linéaire" de $\mathbb{Z}$ au plus proche voisin, et cette propriété (du moins son équivalent sur $\mathbb{R}$ ) jouera un grand rôle dans le développement qui suit.

Dans toute la suite la probabilité $\mu$ et la mesure (tout d'abord régulière et $\sigma$-finie) $\nu$ sont fixées et symétriques comme dans l'introduction. Commençons par quelques remarques classiques (voir par exemple Muckenhoupt [29] pour les inégalités de Hardy).

Lemme 5 Considérons $\nu=\widetilde{\nu}+\widehat{\nu}$ la décomposition de Lebesgue de $\nu$ avec $\widetilde{\nu} \ll \lambda$ et $\widehat{\nu} \perp \lambda$. On a alors $A(\mu, \nu)=A(\mu, \widetilde{\nu})$.

\section{Preuve :}

En récrivant tout en termes des dérivées, il apparaît que (1) est équivalent à

$$
\forall f \in \mathcal{C}(\mathbb{R}), \quad \frac{1}{2} \int\left(\int_{x}^{y} f(u) d u\right)^{2} \mu(d x) \mu(d y) \leq A \nu\left[f^{2}\right]
$$

En remplaçant $f$ par $|f|$, il suffit clairement de ne considérer de telles inégalités que pour les éléments $f$ de $\mathcal{C}_{+}(\mathbb{R})$, l'ensemble des fonctions continues positives sur $\mathbb{R}$. Ensuite une application de résultats classiques d'approximation permet de remplacer $\mathcal{C}_{+}(\mathbb{R})$ par $\mathcal{B}_{+}(\mathbb{R})$, l'ensemble des fonctions boréliennes positives sur $\mathbb{R}$. Détaillons un peu ce point. On cherche donc à montrer que la borne

$$
\forall f \in \mathcal{C}_{+}(\mathbb{R}), \quad \frac{1}{2} \int\left(\int_{x}^{y} f(u) d u\right)^{2} \mu(d x) \mu(d y) \leq A \nu\left[f^{2}\right]
$$

pour un certain $A \in \mathbb{R}_{+}^{*}$ implique que

$$
\forall f \in \mathcal{B}_{+}(\mathbb{R}), \quad \frac{1}{2} \int\left(\int_{x}^{y} f(u) d u\right)^{2} \mu(d x) \mu(d y) \leq A \nu\left[f^{2}\right]
$$

On remarque qu'il suffit d'avoir ces dernières inégalités pour les fonctions $f \in \mathcal{B}_{+}(\mathbb{R})$ telles que $\nu\left[f^{2}\right]<+\infty$ (sinon l'inégalité correspondante ne dit rien), puis par le lemme de Fatou (pour le membre de gauche) et des arguments de convergence dominée (pour le membre de droite), on peut se contenter de considérer de telles fonctions pour lesquelles il existe $0<\epsilon<M$ tel que l'image de $f$ soit incluse dans $\{0\} \sqcup[\epsilon, M]$. Ceci assure notamment que $\nu(\{f>0\})<+\infty$ et par régularité supérieure de $\nu$ on peut trouver un ouvert $U$ tel que $\{f>0\} \subset U$ et $\nu(U)<+\infty$. Soit $\left(g_{n}\right)_{n \in \mathbb{N}}$ une suite de fonctions positives, continues et bornées par $\mathbb{1}_{U}$, convergeant simplement vers $\mathbb{1}_{U}$. Par ailleurs, à nouveau par régularité de $\nu+\lambda$ (voir par exemple le livre de Rudin [39]), on peut trouver une suite $\left(f_{n}\right)_{n \in \mathbb{N}}$ de fonctions positives, continues et bornées par $M$ convergeant $(\nu+\lambda)$ p.p. vers $f$. Ceci reste vrai pour la suite $\left(h_{n}\right)_{n \in \mathbb{N}}:=\left(f_{n} g_{n}\right)_{n \in \mathbb{N}}$, mais on peut appliquer à celle-ci un 
théorème de convergence dominée, puisque pour tout $n \in \mathbb{N},\left|h_{n}\right| \leq M \mathbb{1}_{U}$ et $\nu\left(M \mathbb{1}_{U}\right)<+\infty$. En ayant à nouveau recours au lemme de Fatou pour les membres de gauche, on se rend compte qu'en appliquant (5) aux $h_{n}$, pour $n \in \mathbb{N}$, et en passant à la limite on obtient l'inégalité de (6) pour $f$. Soit maintenant $D \in \mathcal{R}$ tel que $\lambda\left(D^{\mathrm{c}}\right)=0$ et $\widehat{\nu}(D)=0$. En remplaçant $f$ par $f \mathbb{1}_{D}$ dans les inégalités précédentes, on se rend compte que $A(\mu, \nu)$ est aussi l'infimum de l'ensemble des constantes $A \in \mathbb{R}_{+}$ telles que

$$
\begin{aligned}
\forall f \in \mathcal{B}_{+}(\mathbb{R}), \quad \frac{1}{2} \int\left(\int_{x}^{y} f(u) d u\right)^{2} \mu(d x) \mu(d y) & \leq A \nu\left[f^{2} \mathbb{1}_{D}\right] \\
& =A \widetilde{\nu}\left[f^{2}\right]
\end{aligned}
$$

d'où l'identité annoncée $A(\mu, \nu)=A(\mu, \widetilde{\nu})$.

Remarque $6 \mathrm{Il}$ est bien connu que la régularité est la condition permettant de réduire les mesures à leur simple action sur les fonctions continues et dans ce papier, cette hypothèse sur $\nu$ ne sert qu'à justifier le passage de (5) à (6). Ainsi si on définit $A(\mu, \nu)$ comme l'infimum des constantes $A \in \mathbb{R}_{+}$telles que (1) soit plutôt satisfait pour toute fonction $f$ absolument continue $\left(f^{\prime}\right.$ désignant sa dérivée de Radon-Nikodym), alors ce qui suit reste valable sans hypothèse de régularité sur $\nu$ (rappelons que dans (6), il suffit de considérer des fonctions bornées).

Par contre sans l'hypothèse de régularité, les deux définitions de $A(\mu, \nu)$ à partir de (5) et de (6) ne sont pas équivalentes. Par exemple si l'on considère $\mu \neq \delta_{0}$ et $\nu:=\sum_{q \in \mathbb{Q}} \delta_{q}$, la borne (5) est satisfaite pour toute constante $A>0$, puisque le membre de droite est infini sauf pour $f \equiv 0$, d'où $A(\mu, \nu)=0$. Cependant si dans (6) on prend $f=\mathbb{1}_{\mathbb{R} \backslash \mathbb{Q}}$, on aboutit à une contradiction, d'où $A(\mu, \nu)=+\infty$ et c'est cette définition qui est correcte au vu de ce qui va suivre. On supposera donc désormais que $A(\mu, \nu)$ est défini à partir de (6). Dans le même registre, notons que la $\sigma$-finitude de $\nu$ implique l'existence d'une fonction $f \in \mathcal{B}_{+}(\mathbb{R})$ strictement positive telle que $\nu\left[f^{2}\right]<+\infty$, ce qui interdit que $A(\mu, \nu)=0$ si $\mu \neq \delta_{0}$ (ou plus généralement si $\mu$ n'est pas une masse de Dirac dans les cas non symétriques).

Remarque 7 On peut aussi questionner l'hypothèse de $\sigma$-finitude de $\nu$. Son intérêt premier est que la dérivée de Radon-Nikodym $d \nu / d \lambda$ admet bien un sens. Cependant de manière générale, on peut définir la dérivée de Radon-Nikodym d'une mesure $\nu$ quelconque (positive!) par rapport à une mesure $\sigma$-finie $\lambda$ de la manière suivante. Considérons

$$
\mathcal{F}:=\left\{f \in \mathbb{L}_{+}^{0}(\lambda): f \cdot \lambda \leq \nu\right\}
$$

où $\mathbb{L}_{+}^{0}(\lambda)$ est l'ensemble des classes d'équivalence pour la relation d'égalité $\lambda$-p.p. des fonctions à valeurs dans $\overline{\mathbb{R}}_{+}$et où $f \cdot \lambda$ est la mesure admettant $f$ pour densité par rapport à $\lambda$ (ainsi par exemple $(+\infty) \cdot \lambda$ est la mesure définie sur les éléments $A$ de la $\sigma$-algèbre sous-jacente par $((+\infty) \cdot \lambda)(A)=0$ si $\lambda(A)=0$ et $((+\infty) \cdot \lambda)(A)=+\infty$ sinon $)$. Comme tout sous-ensemble de $\mathbb{L}^{0}(\lambda), \mathcal{F}$ admet un supremum dans $\mathbb{L}^{0}(\lambda)$ (voir par exemple le livre de Neveu [30]). Il suffit alors de poser $d \nu / d \lambda$ comme étant ce supremum. Il s'agit en fait d'un maximum. En effet, $\mathcal{F}$ étant stable par maximisation (si $f, g \in \mathcal{F}$, alors $f \vee g \in \mathcal{F}$, on s'en rend compte en considérant séparément les deux événements $\{f \geq g\}$ et $\{f<g\}$ ), on sait (cf. [30]) qu'il existe une suite maximisante $\left(f_{n}\right)_{n \in \mathbb{N}}$ d'éléments de $\mathcal{F}$ convergeant en croissant $\lambda$-p.p. vers $d \nu / d \lambda$ et il en découle facilement que $d \nu / d \lambda \in \mathcal{F}$. Par contre, on ne dispose pas en général de décomposition correspondante de Lebesgue : il n'existe pas de mesure $\widehat{\nu} \perp \lambda$ telle que $\nu=(d \nu / d \lambda) \cdot \lambda+\widehat{\nu}$, comme on peut s'en persuader en prenant pour $\nu$ la mesure de comptage et $\lambda$ la mesure de Lebesgue sur $\mathbb{R}$ (dans cette situation on a $(d \nu / d \lambda)=+\infty$ et si $\widehat{\nu}$ est comme ci-dessus alors nécessairement $\widehat{\nu}=\nu$ qui n'est pas étrangère à $\lambda$...). Revenons à notre contexte et pour une mesure $\nu$ quelconque, posons 
$\widetilde{\nu}=(d \nu / d \lambda) \cdot \lambda$, puis vérifions que l'on a encore $A(\mu, \nu)=A(\mu, \widetilde{\nu})$. L'inégalité $A(\mu, \nu) \leq A(\mu, \widetilde{\nu})$ découle immédiatement de $(d \nu / d \lambda) \cdot \lambda \leq \nu$. Réciproquement, dans (6) il n'est besoin de considérer que des fonctions $f \in \mathcal{B}_{+}(\mathbb{R})$ telles que $\int f^{2} d \nu=\int f^{2} \mathbb{1}_{\{f>0\}} d \nu<+\infty$, ce qui permet de réaliser que $A(\mu, \nu)=\inf _{E \in \mathcal{E}} A\left(\mu, \mathbb{1}_{E} \cdot \nu\right)$, où $\mathcal{E}$ est l'ensemble des boréliens de $\mathcal{R}$ tels que $\mathbb{1}_{E} \cdot \nu$ soit $\sigma$-fini (cette propriété étant équivalente à l'existence d'une fonction strictement positive et intégrable). Pour un tel $E \in \mathcal{E}$, soit $f_{E}$ la dérivée de Radon-Nikodym usuelle de $\mathbb{1}_{E} \cdot \nu$ par rapport à $\lambda$, on a nécessairement $f_{E} \leq(d \nu / d \lambda), \lambda$-p.p. et $A\left(\mu, \mathbb{1}_{E} \cdot \nu\right)=A\left(\mu, f_{E} \cdot \lambda\right)$. De ces deux faits, il ressort que $A\left(\mu, \mathbb{1}_{E} \cdot \nu\right) \geq A(\mu, \widetilde{\nu})$, d'où le résultat annoncé.

Par contre, on peut maintenant avoir $A(\mu, \nu)=0$ sans que $\mu$ soit une masse de Dirac, plus précisément, les développements qui suivent permettent de voir que $A(\mu, \nu)=0$ si et seulement si $d \nu / d \lambda=+\infty \lambda$-p.p. sur l'enveloppe convexe du support de $\mu$ (et dans ce cas si $\mu$ n'est pas une mesure de Dirac, alors l'inégalité de Poincaré n'est pas satisfaite avec $A(\mu, \nu)=0$, i.e. l'infimum en $A \geq 0$ définissant cette constante n'est pas atteint, de par la convention traditionnelle $0(+\infty)=0$ ). Une petite application des remarques précédentes est que le corollaire 3 sera valable sans restriction sur l'ensemble où s'annule $U^{\prime}$.

Ces considérations nous permettent de supposer désormais que $\nu \ll \lambda$.

Rappelons que $M_{\mu} \in \overline{\mathbb{R}}_{+}$est le supremum du support de $\mu$ (défini dans la proposition 2).

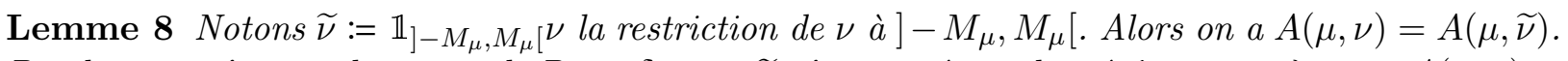

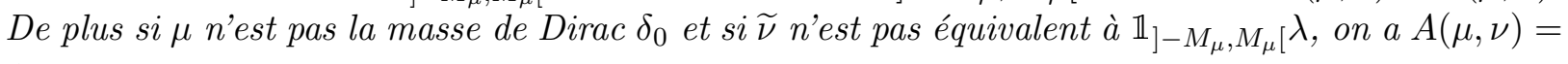
$+\infty$.

\section{Preuve :}

En effet, on ne perd rien dans l'inégalité (6) à n'y considérer que des fonctions $f \in \mathcal{B}_{+}(\mathbb{R})$ qui s'annulent sur $\mathbb{R} \backslash\left[-M_{\mu}, M_{\mu}\right]$, d'où le premier résultat annoncé puisque $\nu \ll \lambda$.

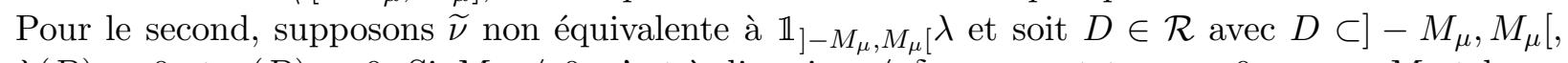
$\lambda(D)>0$ et $\nu(D)=0$. Si $M_{\mu} \neq 0$, c'est-à-dire si $\mu \neq \delta_{0}$, on peut trouver $0<\eta<M_{\mu}$ tel que $D^{\prime}:=D \cap[-\eta, \eta]$ satisfait les mêmes conditions que $D$. On aboutit alors à l'égalité $A(\mu, \nu)=+\infty$ en observant qu'avec $f:=\mathbb{1}_{D^{\prime}}, \operatorname{Var}(f, \mu)>0$ et $\nu\left[f^{2}\right]=0$.

Le cas où $\mu=\delta_{0}$ étant de peu d'intérêt, on supposera dorénavant, sauf mention contraire, que $\mu \neq \delta_{0}$, de sorte que $M_{\mu} \in \mathbb{R}_{+}^{*} \sqcup\{+\infty\}$. Le lemme précédent montre aussi que l'on peut se restreindre à ne considérer que des mesures $\nu$ équivalentes à $\mathbb{1}_{]-M_{\mu}, M_{\mu}[} \lambda$, ce que l'on fera aussi désormais. On peut même encore réduire un peu la classe des mesures $\nu$ pertinentes pour nos problèmes :

Lemme 9 Supposons qu'il existe $0<x<M_{\mu}$ avec

$$
\int_{0}^{x} \frac{1}{\nu(y)} d y=+\infty
$$

(où l'on a étendu l'abus de notation $\nu \hookleftarrow(d \nu) /(d \lambda)$ aux cas envisagés dans la remarque 7). Alors on a $A(\mu, \nu)=+\infty$.

On aura remarqué que dans cette situation on a aussi $B(\mu, \nu)=+\infty$, par définition de $M_{\mu}$.

\section{Preuve :}

Le point $0<x<M_{\mu}$ étant fixé comme ci-dessus, notons pour $a>0, D_{a}:=\{0 \leq y \leq x: \nu(y) \geq$ $1 / a\}$. On calcule qu'avec $f_{a}:=\mathbb{1}_{D_{a}} 1 / \nu$,

$$
\begin{aligned}
\operatorname{Var}(f, \mu) & \geq \int\left(\int_{0}^{x} f_{a}(u) d u\right)^{2} \mathbb{1}_{\{y \leq 0, z \geq x\}} \mu(d y) \mu(d z) \\
& \left.\left.=F^{2}(a) \mu(]-\infty, 0\right]\right) \mu([x,+\infty[)
\end{aligned}
$$


avec

$$
\forall a>0, \quad F(a):=\int_{D_{a}} \frac{1}{\nu(u)} d u
$$

Cependant on a aussi $\nu\left[f_{a}^{2}\right]=F(a)$. Ainsi si $A \in \mathbb{R}_{+}$satisfait l'inégalité (6), on a nécessairement

$$
A \geq F(a) \mu(]-\infty, 0]) \mu([x,+\infty[)
$$

du moins pour les $a>0$ tels que $0<F(a)<+\infty$, ce qui est vrai pour tout $a>0$ assez grand (nos restrictions précédentes interdisant le cas trivial où $\nu$ est identiquement nul). Reste à faire tendre $a$ vers $+\infty$ pour se rendre compte qu'il ne peut pas exister de telle constante $A \in \mathbb{R}_{+}$, puisque $\lim _{a \rightarrow+\infty} F(a)=+\infty$ et $\left.\left.\mu(]-\infty, 0\right]\right) \mu([x,+\infty[)>0$.

Cependant, comme on pourra le retrouver a posteriori grâce aux bornes (2), le lemme précédent n'est plus nécessairement juste si l'on y prend $x=M_{\mu}$. On peut toutefois s'y ramener par le biais des observations suivantes : pour $0<\eta<M_{\mu}$, définissons une constante $A_{\eta}(\mu, \nu)$ de la manière suivante : si $M_{\mu}<+\infty$ (respectivement $\left.M_{\mu}=+\infty\right)$, posons

$$
A_{\eta}(\mu, \nu):=\sup _{f \in \mathcal{D}(\mu, \nu, \eta)} \frac{1}{2 \nu\left[f^{2}\right]} \int\left(\int_{x}^{y} f(u) d u\right)^{2} \mu(d x) \mu(d y)
$$

où $\mathcal{D}(\mu, \nu, \eta)$ est l'ensemble des fonctions $f$ de $\mathcal{B}_{+}(\mathbb{R})$ telles que $\nu\left[f^{2}\right]<+\infty$ et dont le support est inclus dans $\left[-M_{\mu}+\eta, M_{\mu}-\eta\right]$ (resp. dans $\left.[-1 / \eta, 1 / \eta]\right)$. Avec cette définition, il est clair que

$$
\lim _{\eta \rightarrow 0_{+}} A_{\eta}(\mu, \nu)=A(\mu, \nu)
$$

Or pour $0<\eta<M_{\mu}<+\infty$, définissons

$$
\begin{aligned}
\mu_{\eta} & \left.\left.:=\mathbb{1}_{-M_{\mu}+\eta, M_{\mu}-\eta[} \cdot \mu+\mu(]-\infty,-M_{\mu}+\eta\right]\right) \delta_{-M_{\mu}+\eta}+\mu\left(\left[M_{\mu}-\eta,+\infty[) \delta_{M_{\mu}-\eta}\right.\right. \\
\nu_{\eta} & :=\mathbb{1}_{-M_{\mu}+\eta, M_{\mu}-\eta[} \cdot \nu
\end{aligned}
$$

et pour $0<\eta<M_{\mu}=+\infty$,

$$
\begin{aligned}
\mu_{\eta} & \left.\left.:=\mathbb{1}_{]-1 / \eta, 1 / \eta[} \cdot \mu+\mu(]-\infty,-1 / \eta\right]\right) \delta_{-1 / \eta}+\mu\left(\left[1 / \eta,+\infty[) \delta_{1 / \eta}\right.\right. \\
\nu_{\eta} & :=\mathbb{1}_{]-1 / \eta, 1 / \eta[} \cdot \nu
\end{aligned}
$$

de sorte que

Lemme 10 Pour tout $0<\eta<M_{\mu}$, on a $A_{\eta}(\mu, \nu)=A\left(\mu_{\eta}, \nu_{\eta}\right)$.

\section{Preuve :}

Ceci découle immédiatement du fait que pour tout $f \in \mathcal{D}(\mu, \nu, \eta)$, on a

$$
\begin{aligned}
\int\left(\int_{x}^{y} f(u) d u\right)^{2} \mu(d x) \mu(d y) & =\int\left(\int_{x}^{y} f(u) d u\right)^{2} \mu_{\eta}(d x) \mu_{\eta}(d y) \\
\nu\left[f^{2}\right] & =\nu_{\eta}\left[f^{2}\right]
\end{aligned}
$$

Par cette approximation, on se ramène donc à concentrer notre attention sur les couples $(\mu, \nu)$ tels que $M_{\mu}<+\infty$ et $\int_{0}^{M_{\mu}} 1 / \nu(s) \lambda(d s)<+\infty$, ce que nous ferons pour le reste de cette section.

Nous allons maintenant procéder à des régularisations de $\mu$ et $\nu$. Continuons tout d'abord avec $\nu$ en faisant la remarque suivante :

$$
A(\mu, \nu)=\sup _{f \in \mathbb{L}_{+}^{2}\left(\left[-M_{\mu}, M_{\mu}\right], \lambda\right)} \frac{1}{2 \lambda\left[f^{2}\right]} \int\left(\int_{x}^{y} f(u) \sqrt{V_{\nu}(u)} d u\right)^{2} \mu(d x) \mu(d y)
$$


où $V_{\nu} \in \mathbb{L}_{+}^{1}(]-M_{\mu}, M_{\mu}[, \lambda)$ est l'application définie par

$$
\left.V_{\nu}:\right]-M_{\mu}, M_{\mu}\left[\ni x \mapsto \frac{1}{\nu(x)} \in \overline{\mathbb{R}}_{+}\right.
$$

(elle dépend donc aussi légèrement de $\mu$ par l'intermédiaire de $M_{\mu}$, que l'on suppose fixé pour cette discussion).

En effet, pour passer de

$$
A(\mu, \nu)=\sup _{f \in \mathbb{L}_{+}^{2}(\nu)} \frac{1}{2 \nu\left[f^{2}\right]} \int\left(\int_{x}^{y} f(u) d u\right)^{2} \mu(d x) \mu(d y)
$$

à (8), on note que si $D:=\{(d \nu) /(d \lambda)=+\infty\}$ et si $f \in \mathcal{B}_{+}\left(\left[-M_{\mu}, M_{\mu}\right]\right)$ est telle que $\nu\left[f^{2}\right]<+\infty$, alors $f=0 \lambda$-p.p. sur $D$, ainsi $f$ peut s'écrire p.p. sous la forme $g \sqrt{V_{\nu}}$ avec $g \in \mathcal{B}_{+}\left(\left[-M_{\mu}, M_{\mu}\right]\right)$ (rappelons que par nos restrictions précédentes, $\{(d \nu) /(d \lambda)=0\}$ est $\lambda$-négligeable) vérifiant $\lambda\left[g^{2}\right]=$ $\nu\left[f^{2}\right]<+\infty$. Réciproquement si l'on se donne $g \in \mathbb{L}_{+}^{2}\left(\left[-M_{\mu}, M_{\mu}\right], \lambda\right)$, on a bien $g \sqrt{V_{\nu}} \in \mathbb{L}_{+}^{2}(\nu)$ et $\nu\left[g^{2} V_{\nu}\right]=\lambda\left[g^{2}\right]$, d'où en fin de compte (8). Profitons-en pour signaler que l'on peut aussi dans (8) ne considérer que des $f \neq 0$ (dans $\mathbb{L}_{+}^{2}\left(\left[-M_{\mu}, M_{\mu}\right], \lambda\right)$, puisque la contribution de la fonction nulle est $0=0 / 0$.

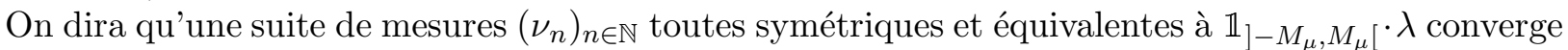
convenablement vers $\nu$, si pour tout $n \in \mathbb{N}, V_{\nu_{n}}$ peut se prolonger en une fonction strictement positive et de classe $\mathcal{C}^{\infty}$ sur un voisinage de $\left[-M_{\mu}, M_{\mu}\right]$ et si les $V_{\nu_{n}}$ convergent pour $n$ grand vers $V_{\nu}$ dans $\mathbb{L}^{1}(]-M_{\mu}, M_{\mu}[, \lambda)$. La régularité de $\mathbb{1}_{]-M_{\mu}, M_{\mu}[} \cdot \lambda$ nous assure sans difficulté de l'existence de telles suites approximantes.

Lemme 11 Supposons que la suite $\left(\nu_{n}\right)_{n \in \mathbb{N}}$ converge convenablement vers $\nu$, alors

$$
\lim _{n \rightarrow \infty} A\left(\mu, \nu_{n}\right)=A(\mu, \nu)
$$

\section{Preuve :}

Soient $V, W \in \mathbb{L}_{+}^{1}(]-M_{\mu}, M_{\mu}[, \lambda)$, on calcule que

$$
\begin{aligned}
\frac{1}{2}\left|\sqrt{\int\left(\int_{x}^{y} f(u) \sqrt{V(u)} d u\right)^{2} \mu(d x) \mu(d y)}-\sqrt{\int\left(\int_{x}^{y} f(u) \sqrt{W(u)} d u\right)^{2} \mu(d x) \mu(d y)}\right|^{2} \\
\leq \frac{1}{2} \int\left(\int_{x}^{y} f(u)(\sqrt{V(u)}-\sqrt{W(u)} d u)^{2} \mu(d x) \mu(d y)\right. \\
\quad=\int_{x \leq y}\left(\int_{x}^{y} f(u)(\sqrt{V(u)}-\sqrt{W(u)}) d u\right)^{2} \mu(d x) \mu(d y) \\
\leq \int_{x \leq y}\left(\int_{x}^{y} f^{2}(u) d u \int_{x}^{y}(\sqrt{V(v)}-\sqrt{W(v)})^{2} d v\right) \mu(d x) \mu(d y) \\
\leq \int_{-M_{\mu}}^{M_{\mu}}(\sqrt{V(v)}-\sqrt{W(v)})^{2} d v \int_{x \leq y}\left(\int_{x}^{y} f^{2}(u) d u\right) \mu(d x) \mu(d y)
\end{aligned}
$$

Cependant en utilisant l'inégalité

$$
\forall t, s \geq 0, \quad(\sqrt{t}-\sqrt{s})^{2} \leq|t-s|
$$

on se rend compte que le premier facteur du membre de droite est majoré par $\|V-W\|_{\mathbb{L}^{1}(]-M_{\mu}, M_{\mu}[, \lambda)}$. Le second est clairement borné par $\int_{-M_{\mu}}^{M_{\mu}} f^{2} d \lambda$ et il apparaît alors que pour tout $n \in \mathbb{N}$ (en 
appliquant ce qui précède avec $V=V_{\nu_{n}}$ et $W=V_{\nu}$ ),

$$
\begin{aligned}
& \left|\sqrt{A\left(\mu, \nu_{n}\right)}-\sqrt{A(\mu, \nu)}\right| \\
& \leq \sup _{f \in \mathcal{B}_{+}\left(\left[-M_{\mu}, M_{\mu}\right]\right): 0<\lambda\left[f^{2}\right]<+\infty} \mid \sqrt{\frac{1}{2 \lambda\left[f^{2}\right]} \int\left(\int_{x}^{y} f(u) \sqrt{V_{\nu_{n}}(u)} d u\right)^{2} \mu(d x) \mu(d y)} \\
& -\sqrt{\frac{1}{2 \lambda\left[f^{2}\right]} \int\left(\int_{x}^{y} f(u) \sqrt{V_{\nu}(u)} d u\right)^{2} \mu(d x) \mu(d y)} \\
& =\sup _{f \in \mathcal{B}_{+}\left(\left[-M_{\mu}, M_{\mu}\right]\right): 0<\lambda\left[f^{2}\right]<+\infty} \frac{1}{\sqrt{2 \lambda\left[f^{2}\right]}} \mid \sqrt{\int\left(\int_{x}^{y} f(u) \sqrt{V_{\nu_{n}}(u)} d u\right)^{2} \mu(d x) \mu(d y)} \\
& -\sqrt{\int\left(\int_{x}^{y} f(u) \sqrt{V_{\nu}(u)} d u\right)^{2} \mu(d x) \mu(d y)} \\
& \leq \sqrt{\left\|V_{\nu_{n}}-V_{\nu}\right\|_{\mathbb{L}^{1}(]-M_{\mu}, M_{\mu}[, \lambda)}}
\end{aligned}
$$

On est donc ramené à l'estimation de $A(\mu, \nu)$ pour des mesures $\nu$ telles que $V_{\nu}$ soit strictement positif et de classe $\mathcal{C}^{\infty}$ sur $\left[-M_{\mu}, M_{\mu}\right]$. Montrons que l'on peut aussi ne considérer que des probabilités $\mu$ admettant une densité de classe $\mathcal{C}^{\infty}$ et strictement positive sur $\left[-M_{\mu}, M_{\mu}\right]$. Pour cela notons $\mathcal{D}(\mu)$ l'ensemble de ces probabilités, de plus supportées par $\left[-M_{\mu}, M_{\mu}\right]$ et symétriques (pour tout $\mu^{\prime} \in \mathcal{D}(\mu)$ on a donc $\left.M_{\mu^{\prime}}=M_{\mu}\right)$. On dira qu'une suite $\left(\mu_{n}\right)_{n \in \mathbb{N}}$ d'éléments de $\mathcal{D}(\mu)$ converge adéquatement vers $\mu$ si $F_{\mu_{n}}$ converge vers $F_{\mu}$ dans $\mathbb{L}^{1}(]-M_{\mu}, M_{\mu}[, \lambda)$, où rappelons que $F_{\mu}$ est la fonction de répartition de $\mu$, que l'on peut restreindre à $\left[-M_{\mu}, M_{\mu}\right]$, car avant elle vaut 0 et 1 après. Puisqu'une fonction de répartition est à valeurs dans $[0,1]$, la convergence $\mathbb{L}^{1}$ ci-dessus est équivalente à la convergence $\mathbb{L}^{p}$, pour n'importe quel $1 \leq p<+\infty$. On a même beaucoup mieux :

Lemme 12 Soit $-\infty \leq M<M^{\prime} \leq+\infty$ et $\left(F_{n}\right)_{n \in \mathbb{N}}$ une suite de fonctions croissantes sur $] M, M^{\prime}[$ convergeant dans $\mathbb{L}^{1}(] M, M^{\prime}[, \lambda)$ vers une fonction $F$. Alors $F$ admet une $\lambda$-modification croissante et en tout point $u \in] M, M^{\prime}\left[\right.$ de continuité pour celle-ci, on a $\lim _{n \rightarrow \infty} F_{n}(u)=F(u)$. De même pour les inverses généralisés, si l'on fait l'hypothèse que les enveloppes convexes des $F_{n}(] M, M^{\prime}[)$, pour $n \in \mathbb{N}$, coïncident toutes avec l'enveloppe convexe de $F(] M, M^{\prime}[$ ) (éventuellement aux points extrémaux près); si $u \in F(] M, M^{\prime}[)$ est un point de continuité de $F^{-1}$, alors $\lim _{n \rightarrow \infty} F_{n}^{-1}(u)=$ $F^{-1}(u)$.

Ce résultat de renforcement de la qualité de la convergence peut être vu comme un équivalent pour la convergence p.p. du second théorème de Dini.

\section{Preuve :}

Il est bien connu que d'une convergence $\mathbb{L}^{1}$ on peut extraire une sous-suite convergeant p.p. Soit $\mathcal{N}$ l'ensemble $\lambda$-négligeable sous-jacent. On a donc que $F$ est croissante sur $] M, M^{\prime}[\backslash \mathcal{N}$. Il suffit alors de redéfinir pour tout $x \in \mathcal{N}, F(x) \hookleftarrow \inf \{F(y): y \in] s, M[\backslash \mathcal{N}\}$ pour obtenir une $\lambda$-modification de $F$ croissante.

Soit ensuite $u \in] M, M^{\prime}[$ un point de continuité pour cette dernière et donnons-nous $\epsilon>0$. On peut donc trouver $\delta>0$ tel que $] u-\delta, u+\delta[\subset] M, M^{\prime}[$ et $F(] u-\delta, u+\delta[) \subset] F(u)-\epsilon / 3, F(u)+\epsilon / 3[$. Supposons maintenant que $\lim _{\sup _{n \rightarrow \infty}} F_{n}(u) \geq F(u)+\epsilon$. Quitte à extraire une sous-suite de $\left(F_{n}\right)_{n \in \mathbb{N}}$, que l'on renote de la même manière, on peut faire l'hypothèse que pour tout $n$ assez grand, $F_{n}(u) \geq F(u)+2 \epsilon / 3$. Mais alors on calcule que pour ces $n$,

$$
\begin{aligned}
\left\|F_{n}-F\right\|_{\mathbb{L}^{1}(] M, M^{\prime}[, \lambda)} & \geq \int_{u}^{u+\delta} F_{n}(v)-F(v) d v \\
& \geq \delta \epsilon / 3
\end{aligned}
$$


par croissance de $F_{n}$ sur $[u, u+\delta[$. On aboutit ainsi à une contradiction avec la convergence de la suite $\left(F_{n}\right)_{n \in \mathbb{N}}$ vers $F$ dans $\mathbb{L}^{1}(] M, M^{\prime}[, \lambda)$. On rencontre le même problème si l'on suppose que $\liminf _{n \rightarrow \infty} F_{n}(u) \leq F(u)-\epsilon$, en intégrant plutôt sur $\left.] u-\delta, u\right]$. D'où la première convergence simple annoncée.

Pour la convergence correspondante des inverses généralisés, on pourrait aussi s'en convaincre à partir de manipulations similaires, mais le plus simple est de faire un dessin. Par symétrie par rapport à la diagonale, on voit que pour tout $n \in \mathbb{N}$,

$$
\left\|F_{n}-F\right\|_{\mathbb{L}^{1}(] M, M^{\prime}[, \lambda)}=\left\|F_{n}^{-1}-F^{-1}\right\|_{\mathbb{L}^{1}(] I, I^{\prime}[, \lambda)}
$$

car il s'agit de l'aire "située à l'intérieur des deux graphes de $F_{n}$ et de $F$ (en reliant d'un trait les sauts)", où $] I, I^{\prime}\left[\right.$ est l'intérieur de l'enveloppe convexe de $F(] M, M^{\prime}[)$. Le second résultat de convergence simple découle alors du premier.

Revenant à notre contexte, on en déduit que si la suite $\left(\mu_{n}\right)_{n \in \mathbb{N}}$ de $\mathcal{D}(\mu)$ converge adéquatement vers $\mu$, alors pour $n$ grand $F_{\mu_{n}}$ converge p.p. vers $F_{\mu}$ sur ] $-M_{\mu}, M_{\mu}$ [ et $F_{\mu_{n}}^{-1}$ converge p.p. et dans $\mathbb{L}^{1}(] 0,1[, \lambda)$ vers $F_{\mu}^{-1}$ sur $] 0,1[$. C'est surtout cette dernière convergence qui sera utile dans la section 4 , mais ici contentons-nous d'indiquer que toute probabilité $\mu \neq \delta_{0}$ peut être adéquatement approchée et prouvons la version correspondante du lemme 11 :

Lemme 13 Supposons que la suite $\left(\mu_{n}\right)_{n \in \mathbb{N}}$ de $\mathcal{D}(\mu)$ converge adéquatement vers $\mu$, alors

$$
\lim _{n \rightarrow \infty} A\left(\mu_{n}, \nu\right)=A(\mu, \nu)
$$

(où $\nu$ est suffisamment régulier pour que $V_{\nu}$ soit borné sur $\left[-M_{\mu}, M_{\mu}\right]$ ).

\section{Preuve :}

Soient $f \in \mathbb{L}^{2}\left(\left[-M_{\mu}, M_{\mu}\right], \lambda\right)$ et $\widetilde{\mu}$ une probabilité à support dans $\left[-M_{\mu}, M_{\mu}\right]$, par le théorème de Fubini, on peut écrire

$$
\begin{aligned}
\int_{x \leq y} & \left(\int_{x}^{y} f(u) \sqrt{V_{\nu_{n}}(u)} d u\right)^{2} \widetilde{\mu}(d x) \widetilde{\mu}(d y) \\
= & \int_{x \leq y}\left(\int_{x}^{y} f(u) \sqrt{V_{\nu_{n}}(u)} d u\right)\left(\int_{x}^{y} f(v) \sqrt{V_{\nu_{n}}(v)} d v\right) \widetilde{\mu}(d x) \widetilde{\mu}(d y) \\
= & \int_{]-M_{\mu}, M_{\mu}\left[^{2}\right.} d u d v f(u) \sqrt{V_{\nu_{n}}(u)} f(v) \sqrt{V_{\nu_{n}}(v)} \int \mathbb{1}_{x \leq u \wedge v, u \vee v<y} \widetilde{\mu}(d y) \widetilde{\mu}(d x) \\
= & \left.\left.\int_{]-M_{\mu}, M_{\mu}\left[^{2}\right.} d u d v f(u) \sqrt{V_{\nu_{n}}(u)} f(v) \sqrt{V_{\nu_{n}}(v) \widetilde{\mu}}\left(\left[-M_{\mu}, u \wedge v\right]\right) \widetilde{\mu}(] u \vee v, M_{\mu}\right]\right) \\
= & \int_{]-M_{\mu}, M_{\mu}\left[^{2}\right.} f(u) \sqrt{V_{\nu_{n}}(u)} f(v) \sqrt{V_{\nu_{n}}(v)} G_{\widetilde{\mu}}(u, v) \lambda^{\otimes 2}(d u, d v)
\end{aligned}
$$

où l'on a été amené à poser

$$
\forall(u, v) \in]-M_{\mu}, M_{\mu}\left[^{2}, \quad G_{\widetilde{\mu}}(u, v):=F_{\widetilde{\mu}}(u \wedge v)\left(1-F_{\widetilde{\mu}}(u \vee v)\right)\right.
$$

(on aura remarqué que si l'on avait remplacé $F_{\widetilde{\mu}}(u \vee v)$ par $F_{\widetilde{\mu}}(u \vee v-)$ dans la définition ci-dessus, cela n'aurait rien changé aux intégrales précédentes).

On a donc

$$
\begin{aligned}
& \left|\int_{x \leq y}\left(\int_{x}^{y} f(u) \sqrt{V_{\nu_{n}}(u)} d u\right)^{2} \widetilde{\mu}(d x) \widetilde{\mu}(d y)-\int_{x \leq y}\left(\int_{x}^{y} f(u) \sqrt{V_{\nu_{n}}(u)} d u\right)^{2} \mu(d x) \mu(d y)\right| \\
& =\left|\int_{]-M_{\mu}, M_{\mu} l^{2}} f(u) \sqrt{V_{\nu_{n}}(u)} f(v) \sqrt{V_{\nu_{n}}(v)}\left(G_{\widetilde{\mu}}(u, v)-G_{\mu}(u, v)\right) \lambda^{\otimes 2}(d u, d v)\right|
\end{aligned}
$$




$$
\begin{aligned}
& \leq\left\|V_{\nu}\right\|_{\infty} \int_{]-M_{\mu}, M_{\mu}\left[^{2}\right.} f(u) f(v)\left|G_{\widetilde{\mu}}(u, v)-G_{\mu}(u, v)\right| \lambda^{\otimes 2}(d u, d v) \\
& \leq\left\|V_{\nu}\right\|_{\infty} \sqrt{\int_{]-M_{\mu}, M_{\mu}\left[^{2}\right.} f^{2}(u) f^{2}(v) \lambda^{\otimes 2}(d u, d v)} \sqrt{\int_{]-M_{\mu}, M_{\mu}\left[^{2}\right.}\left(G_{\widetilde{\mu}}(u, v)-G_{\mu}(u, v)\right)^{2} \lambda^{\otimes 2}(d u, d v)} \\
& =\left\|V_{\nu}\right\|_{\infty} \lambda\left[f^{2} \mathbb{1}_{]-M_{\mu}, M_{\mu}[}\right]\left\|G_{\widetilde{\mu}}-G_{\mu}\right\|_{\mathbb{L}^{2}(]-M_{\mu}, M_{\mu}\left[^{2}, \lambda^{\otimes 2}\right)}
\end{aligned}
$$

On pourra alors conclure comme dans la preuve du lemme 11 s'il est possible de bien majorer $\left\|G_{\widetilde{\mu}}-G_{\mu}\right\|_{\mathbb{L}^{2}(]-M_{\mu}, M_{\mu}\left[^{2}, \lambda \otimes 2\right)}$ en termes de $\left\|F_{\widetilde{\mu}}-F_{\mu}\right\|_{\mathbb{L}^{1}(]-M_{\mu}, M_{\mu}[, \lambda)}$. Mais on note que pour tout $(u, v) \in]-M_{\mu}, M_{\mu}\left[^{2}\right.$,

$$
\begin{aligned}
& \left|G_{\widetilde{\mu}}(u, v)-G_{\mu}(u, v)\right| \\
& \quad \leq\left|F_{\widetilde{\mu}}(u \wedge v)-F_{\mu}(u \wedge v)\right|\left(1-F_{\widetilde{\mu}}(u \vee v)\right)+F_{\mu}(u \wedge v)\left|F_{\widetilde{\mu}}(u \vee v)-F_{\mu}(u \vee v)\right| \\
& \quad \leq\left|F_{\widetilde{\mu}}(u \wedge v)-F_{\mu}(u \wedge v)\right|+\left|F_{\widetilde{\mu}}(u \vee v)-F_{\mu}(u \vee v)\right|
\end{aligned}
$$

d'où

$$
\begin{aligned}
\left.\left\|G_{\widetilde{\mu}}-G_{\mu}\right\|_{\mathbb{L}^{2}(]-M_{\mu}, M_{\mu}\left[^{2}, \lambda \otimes 2\right.}^{2}\right) \\
\quad \leq 2\left(\int_{]-M_{\mu}, M_{\mu}\left[^{2}\right.}\left(F_{\widetilde{\mu}}(u \wedge v)-F_{\mu}(u \wedge v)\right)^{2} d u d v+\int_{]-M_{\mu}, M_{\mu}\left[^{2}\right.}\left(F_{\widetilde{\mu}}(u \vee v)-F_{\mu}(u \vee v)\right)^{2} d u d v\right) \\
=\int_{]-M_{\mu}, M_{\mu}[}\left(F_{\widetilde{\mu}}(u)-F_{\mu}(u)\right)^{2}(1-u) d u+\int_{]-M_{\mu}, M_{\mu}[}\left(F_{\widetilde{\mu}}(u)-F_{\mu}(u)\right)^{2} u d u \\
=\int_{]-M_{\mu}, M_{\mu}[}\left(F_{\widetilde{\mu}}(u)-F_{\mu}(u)\right)^{2} d u \\
\leq\left\|F_{\widetilde{\mu}}-F_{\mu}\right\|_{\mathbb{L}^{1}(]-M_{\mu}, M_{\mu}[, \lambda)}
\end{aligned}
$$

puis la convergence voulue.

On se ramène ainsi à des couples $(\mu, \nu)$ très réguliers : $\mu$ admet une densité strictement positive et $\mathcal{C}^{\infty}$ sur $\left[-M_{\mu}, M_{\mu}\right]$ et $V_{\nu}$ vérifie aussi cette propriété. Ceci correspond à la situation envisagée par Bobkov et Götze dans [4]. Notre objectif est d'obtenir (à la fin de la section suivante) pour ces couples une nouvelle description de $A(\mu, \nu)$ puis de montrer que celle-ci est stable par les deux dernières approximations considérées, ce qui permettra de l'étendre au moins à tous les couples $(\mu, \nu)$ vérifiant $M_{\mu}<+\infty$ et $\int_{0}^{M_{\mu}} 1 / \nu d \lambda<+\infty$.

Pour effectuer une réduction "à la Bobkov et Götze" à des problèmes de Sturm-Liouville, nous allons tout d'abord utiliser, comme annoncé, une méthode de chemin.

L'idée est très simple : on se donne $f \in \mathbb{L}_{+}^{2}(]-M_{\mu}, M_{\mu}[, \lambda)$ et $-M_{\mu}<x<y<M_{\mu}$ et on cherche à majorer $\left(\int_{x}^{y} f(u) \sqrt{V_{\nu}(u)} d u\right)^{2}$ linéairement en termes de $\left(f^{2}(v)\right)_{-M_{\mu}<v<M_{\mu}}$. Evidemment ceci va être fait par le biais de l'inégalité de Cauchy-Schwartz et pour nous donner un peu de latitude, considérons de plus une fonction $g$ mesurable et strictement positive sur $[x, y]$. On peut alors écrire

$$
\left(\int_{x}^{y} f(u) \sqrt{V_{\nu}(u)} d u\right)^{2} \leq \int_{x}^{y} f^{2}(u) \frac{1}{g(u)} d u \int_{x}^{y} V_{\nu}(v) g(v) d v
$$

Il est important de remarquer que nous n'avons rien perdu lors de cette opération, puisque le cas d'égalité correspond au choix $g=f V_{\nu}^{-1 / 2}$, indépendamment de $-M_{\mu}<x<y<M_{\mu}$. Il semble donc intéressant de se donner une fonction $g$ mesurable et strictement positive sur $\left[-M_{\mu}, M_{\mu}\right]$ et de procéder à la majoration suivante :

$$
\int_{x \leq y}\left(\int_{x}^{y} f(u) \sqrt{V_{\nu}(u)} d u\right)^{2} \mu(d x) \mu(d y)
$$




$$
\begin{aligned}
& \leq \int_{x \leq y}\left(\int_{x}^{y} f^{2}(u) \frac{1}{g(u)} d u \int_{x}^{y} V_{\nu}(v) g(v) d v\right) \mu(d x) \mu(d y) \\
& =\int_{-M_{\mu}}^{M_{\mu}} d u f^{2}(u) \frac{1}{g(u)} \int_{x \leq u \leq y}\left(\int_{x}^{y} V_{\nu}(v) g(v) d v\right) \mu(d x) \mu(d y)
\end{aligned}
$$

Puisque l'on cherche à comparer ceci à $\int_{-M_{\mu}}^{M_{\mu}} f^{2}(u) d u$, d'une certaine manière la meilleure chose qui puisse nous arriver est que l'expression

$$
\frac{1}{g(u)} \int_{x \leq u \leq y}\left(\int_{x}^{y} V_{\nu}(v) g(v) d v\right) \mu(d x) \mu(d y)
$$

soit $\lambda$-p.p. constant en $u \in]-M_{\mu}, M_{\mu}[$. En notant $A>0$ cette éventuelle constante, on est ainsi amené à se demander s'il existe une solution $g$ strictement positive sur ] $-M_{\mu}, M_{\mu}$ [ à l'équation intégrale

$$
A g(u)=\int_{x \leq u \leq y}\left(\int_{x}^{y} V_{\nu}(v) g(v) d v\right) \mu(d x) \mu(d y)
$$

Si une telle solution existe et est de classe $\mathcal{C}^{2}$ sur $]-M_{\mu}, M_{\mu}$ [, nos conditions de régularité nous permettent de dériver deux fois l'identité précédente pour trouver que $g$ est en fait une solution de l'équation de Sturm-Liouville

$$
A\left(\frac{g^{\prime}}{\mu}\right)^{\prime}=-V_{\nu} g
$$

Puisque l'on cherche une solution strictement positive sur $]-M_{\mu}, M_{\mu}[$, on imagine que le cas critique (en $A$ ) correspond aux conditions de Dirichlet, c'est-à-dire quand $g$ se prolonge par continuité à $\left[-M_{\mu}, M_{\mu}\right]$ avec $g\left(-M_{\mu}\right)=g\left(M_{\mu}\right)=0$.

Pour justifier ce développement heuristique, supposons donc que $A>0$ est tel qu'il existe une solution $g$ strictement positive de (11) sur ] $-M_{\mu}, M_{\mu}$ [. L'ellipticité (i.e. le fait que $0<\min _{\left[-M_{\mu}, M_{\mu}\right]} \mu \leq$ $\left.\max _{\left[-M_{\mu}, M_{\mu}\right]} \mu<+\infty\right)$ et la régularité des coefficients de cette équation font que même si la solution $g$ est comprise au sens faible des distributions, elle est en fait de classe $\mathcal{C}^{\infty}$ sur $\left[-M_{\mu}, M_{\mu}\right]$ (comme précédemment ceci signifie qu'elle peut se prolonger sur un voisinage de ce compact en une fonction de classe $\left.\mathcal{C}^{\infty}\right)$. Par contre elle peut éventuellement s'annuler en $-M_{\mu}$ et $M_{\mu}$. En intégrant (11), il apparaît que pour tous $-M_{\mu} \leq x \leq y \leq M_{\mu}$,

$$
A\left(\frac{g^{\prime}(y)}{\mu(y)}-\frac{g^{\prime}(x)}{\mu(x)}\right)=-\int_{x}^{y} V_{\nu}(u) g(u) d u
$$

Soit $-M_{\mu} \leq t \leq M_{\mu}$ fixé, en intégrant ceci par rapport à $\mathbb{1}_{\{x \leq t \leq y\}} \mu(x) \mu(d y)$ on obtient

$$
\begin{array}{r}
\left.\left.A\left(g\left(M_{\mu}\right)-g(t)\right) \mu\left(\left[-M_{\mu}, t\right]\right)-A\left(g(t)-g\left(-M_{\mu}\right)\right) \mu(] t, M_{\mu}\right]\right) \\
=-\int_{x \leq t \leq y}\left(\int_{x}^{y} V_{\nu}(u) g(u) d u\right) \mu(x) \mu(d y)
\end{array}
$$

c'est-à-dire que pour tout $-M_{\mu} \leq t \leq M_{\mu}$ on a

$$
A g(t)=a(t)+b(t)+\int_{x \leq t \leq y}\left(\int_{x}^{y} V_{\nu}(u) g(u) d u\right) \mu(x) \mu(d y)
$$

avec $\left.\left.a(t):=A g\left(-M_{\mu}\right) \mu(] t, M_{\mu}\right]\right)$ et $b(t):=A g\left(M_{\mu}\right) \mu\left(\left[-M_{\mu}, t\right]\right)$. Puisque ces deux nombres sont positifs, on a

$$
A g(t) \geq \int_{x \leq t \leq y}\left(\int_{x}^{y} V_{\nu}(u) g(u) d u\right) \mu(x) \mu(d y)
$$


En reportant cette inégalité dans (10), on obtient que pour tout $f \in \mathbb{L}^{2}(]-M_{\mu}, M_{\mu}[$ ),

$$
\int_{x \leq y}\left(\int_{x}^{y} f(u) \sqrt{V_{\nu}(u)} d u\right)^{2} \mu(d x) \mu(d y) \leq A \int_{-M_{\mu}}^{M_{\mu}} f^{2}(u) d u
$$

d'où $A(\mu, \nu) \leq A$.

Si de plus $g$ satisfait la condition de Dirichlet, on a pour tout $t \in\left[-M_{\mu}, M_{\mu}\right], a(t)=0=b(t)$, et si l'on prend $f=g \sqrt{V_{\nu}}$, toutes les inégalités précédentes sont des égalités et on aboutit à la conclusion que $A(\mu, \nu)=A$. Il ne peut donc exister qu'un seul $A>0$ tel que (11) admette une solution strictement positive sur $]-M_{\mu}, M_{\mu}$ [ avec condition au bord de Dirichlet et c'est nécessairement $A(\mu, \nu)$. Reste à se persuader de l'existence d'un tel $A>0$. Pour cela, rappelons que par le théorème de Cauchy-Lipschitz une solution de (11) est uniquement déterminée par $g(0)$ et $g^{\prime}(0)$. Puisque l'on cherche des solutions strictement positives sur $]-M_{\mu}, M_{\mu}$, on peut supposer que $g(0)=1$ et par symétrie du problème (condition de Dirichlet au bord incluse), on peut deviner que si elle existe, notre solution vérifiera $g^{\prime}(0)=0$. Considérons donc $g_{A}$ la solution de (11) satisfaisant à ces conditions en 0 . Pour plus de commodité, étendons $\mu$ et $V_{\nu}$ à tout $\mathbb{R}$ en des fonctions $\mathcal{C}^{\infty}$, symétriques, uniformément minorées et majorées par des constantes strictement positives et faisons de sorte que leur deux premières dérivées soient également uniformément bornées (on peut par exemple les rendre périodiques avec une période un peu plus grande que $2 M_{\mu}$ ). L'équation (11) admet alors une unique solution sur tout $\mathbb{R}$ prolongeant $g_{A}$ et on la note de la même manière. Grâce aux théorèmes de comparaison de Sturm (voir par exemple le théorème XI.3.1 du livre [19] de Hartman), on peut comparer la position des zéros de $g_{A}$ avec ceux de solutions d'équations de Sturm-Liouville à coefficients constants. Ceci permet de voir que $g_{A}$ va admettre un premier zéro $0<z(A)<+\infty$ à droite de 0 et que $\lim _{A \rightarrow 0_{+}} z(A)=0$ et $\lim _{A \rightarrow+\infty} z(A)=+\infty$. Notre problème est de savoir s'il existe $A>0$ tel que $z(A)=M_{\mu}$ (puisque tout se passe de manière symétrique sur $\mathbb{R}_{-}$). Une nouvelle application du théorème de comparaison de Sturm montre que l'application $\mathbb{R}_{+}^{*} \ni A \mapsto z(A)$ est strictement croissante. Il suffit donc de voir que cette fonction $z$ est continue, mais ceci découle facilement d'un résultat classique de continuité de l'application $\mathbb{R}_{+}^{*} \ni A \mapsto g_{A}$ si on la considère comme à valeurs dans $\mathcal{C}(\mathbb{R})$ muni de la convergence uniforme sur les compacts (le plus simple pour s'en convaincre est peut-être de transformer (11) en un système linéaire de deux équations différentielles du premier ordre et d'appliquer à une norme de $\left(g_{A}, g_{A}^{\prime}\right)-\left(g_{\widetilde{A}}, g_{\widetilde{A}}^{\prime}\right)$ un lemme de Gronwall, pour $\widetilde{A}>0$ proche de $A>0$ ).

On a donc démontré le résultat suivant.

Proposition $14 \mathrm{Il}$ existe un unique $A>0$ tel que (11) admette une solution strictement positive sur $]-M_{\mu}, M_{\mu}$ [ et s'annulant sur la frontière. Il s'agit de la constante $A(\mu, \nu)$.

Bobkov et Götze [4] se ramènent aussi à considérer sur $\mathbb{R}_{+}$l'équation de Sturm-Liouville (11) avec condition initiale $g(0)=1$ et $g^{\prime}(0)=0$ et à en estimer le premier zéro. Il existe toutefois des petites différences entre nos développements - ces auteurs sont partis des inégalités de Hardy et non pas directement de celles de Poincaré (mais les calculs de [4] et les précédents permettent de voir à quel point elles sont très fortement liées, du moins dans un contexte symétrique, ce qui était un peu prévisible) - Bobkov et Götze ont préféré avoir recours à des considérations spectrales (du type de celles évoquées un peu plus loin) plutôt qu'à l'aspect optimal des méthodes de chemins et aux théorèmes de comparaison de Sturm.

Remarque 15 La proposition 14 reste valable sans aucune hypothèse de symétrie, il suffit d'adapter un peu les arguments précédents en ayant recours à la précision qu'offrent les théorèmes de comparaison de Sturm en termes de la "dérivée initiale". D'ailleurs ce résultat serait déjà inclus tel quel dans la section XI.4 de [19], si au lieu de (11), on s'intéressait à l'équation de Sturm-Liouville $\left(\frac{g^{\prime}}{\mu}\right)^{\prime}=-\left(V_{\nu}+1 / A\right) g$.

Sur un intervalle non vide ]N, $M$ [, on cherche donc à trouver une solution $g$ strictement positive de (11) avec condition de Dirichlet au bord. Comme précédemment, on peut faire l'hypothèse que $\mu$ et $V_{\nu}$ sont des fonctions de classe $\mathcal{C}^{\infty}$ sur $\mathbb{R}$, uniformément minorées et majorées par des 
constantes strictement positives et leur deux premières dérivées sont aussi uniformément bornées. Posons $P:=(N+M) / 2$ et pour $A \in \mathbb{R}_{+}^{*}$ et $a \in \mathbb{R}$, considérons $g$ l'unique solution de (11) sur $\mathbb{R}$ satisfaisant $g(P)=1$ et $g^{\prime}(P)=a$. Notons $z_{+}(A, a)$ et $z_{-}(A, a)$ respectivement le premier zéro de $g$ à droite et à gauche de $P$, ils existent par les mêmes arguments que ci-dessus. Les théorèmes de comparaison de Sturm permettent aussi d'avoir des renseignements sur le comportement de ces points en fonction de $a \in \mathbb{R}$. Ainsi à $A>0$ fixé, les applications $\mathbb{R} \ni a \mapsto z_{+}(A, a)$ et $\mathbb{R} \ni a \mapsto z_{-}(A, a)$ sont strictement croissantes (cf. [19], on a même un peu mieux : pour les couples $(A, a),(\widetilde{A}, \widetilde{a}) \in \mathbb{R}_{+}^{*} \times \mathbb{R}$, tels que $A \leq \widetilde{A}$ et $A a<\widetilde{A} \widetilde{a}$, on est assuré de $\left.z_{+}(A, a)<z_{+}(\widetilde{A}, \widetilde{a})\right)$. De plus, elles sont continues, comme on s'en rend compte par une application du théorème classique de continuité des solutions (toujours dans $\mathcal{C}(\mathbb{R})$ muni de la convergence uniforme sur les compacts) en fonction des conditions initiales. Ce résultat est aussi basé sur un lemme de Gronwall, qui appliqué directement donne plus précisément la continuité conjointe en leur deux arguments de $z_{+}$et de $z_{-}$. Par ailleurs la comparaison avec la solution d'une équation de Sturm-Liouville à coefficients constants permet de voir qu'uniformément en $A$ variant dans un compact de $\mathbb{R}_{+}^{*}$, on a

$$
\lim _{a \rightarrow-\infty} z_{+}(A, a)=P \quad \text { et } \quad \lim _{a \rightarrow+\infty} z_{-}(A, a)=P
$$

On en déduit que pour tout $A>0$ fixé, il existe un unique $a(A) \in \mathbb{R}$ tel que

$$
P-z_{-}(a(A), A)=z_{+}(a(A), A)-P
$$

Notons que l'application $a: \mathbb{R}_{+}^{*} \ni A \mapsto a(A) \in \mathbb{R}$ est continue : soit $\left(A_{n}\right)_{n \in \mathbb{N}}$ une suite d'éléments de $\mathbb{R}_{+}^{*}$ convergeant vers $A \in \mathbb{R}_{+}^{*}$, telle que la suite $\left(a\left(A_{n}\right)\right)_{n \in \mathbb{N}}$ converge dans $\overline{\mathbb{R}}$. Si sa limite devait être $+\infty$, en utilisant d'une part les convergences uniformes (12) et d'autre part (13), on aboutirait à

$$
\lim _{n \rightarrow+\infty} z_{+}\left(a\left(A_{n}\right), A_{n}\right)=P
$$

Cependant une comparaison avec des équations de Sturm-Liouville à coefficients constants indique que pour $A>0$,

$$
\liminf _{(\widetilde{a}, \widetilde{A}) \rightarrow(+\infty, A)} z_{+}(\widetilde{a}, \widetilde{A})>0
$$

d'où une contradiction. De même on montre que $\lim _{n \rightarrow \infty} a\left(A_{n}\right)>-\infty$. Désignons par $a \in \mathbb{R}$ cette limite. Du fait que $z_{+}$et $z_{-}$sont continues, on aura $P-z_{-}(a, A)=z_{+}(a, A)-P$, d'où en fin de compte $a(A)=a$ et la continuité annoncée. Par composition, l'application $\mathbb{R}_{+}^{*} \ni A \mapsto z_{+}(a(A), A)-$ $P$ est donc aussi continue. Ceci permettra de trouver un $A>0$ tel que $z_{-}(a(A), A)=N$ et $z_{+}(a(A), A)=M$, c'est-à-dire tel que (11) admette une solution strictement positive sur $] N, M[$ avec condition de Dirichlet au bord, si l'on arrive à se convaincre que

$$
\begin{aligned}
\lim _{A \rightarrow 0_{+}} z_{+}(a(A), A) & =P \\
\lim _{A \rightarrow+\infty} z_{+}(a(A), A) & =+\infty
\end{aligned}
$$

Mais soit $\widetilde{g}$ la solution de $(11)$ correspondant à la condition initiale $\widetilde{g}(P)=1$ et $\widetilde{g}^{\prime}(P)=0$ et dénotons par $\widetilde{z}_{-}(A)$ et $\widetilde{z}_{+}(A)$ respectivement le premier zéro de $\widetilde{g}$ à droite et à gauche de $P$. Grâce à une nouvelle comparaison avec une équation de Sturm-Liouville à coefficients constant, on obtient

$$
\begin{array}{r}
\lim _{A \rightarrow 0_{+}} \widetilde{z}_{+}(A)=P=\lim _{A \rightarrow 0_{+}} \widetilde{z}_{-}(A) \\
\lim _{A \rightarrow+\infty} \widetilde{z}_{+}(A)=+\infty=-\lim _{A \rightarrow+\infty} \widetilde{z}_{-}(A)
\end{array}
$$

On peut alors conclure aux convergence voulues, car par construction de $a(A)$, il apparait facilement que

$$
\left|\widetilde{z}_{-}(A)-P\right| \wedge\left|\widetilde{z}_{+}(A)-P\right| \leq z_{+}(a(A), A)-P \leq\left|\widetilde{z}_{-}(A)-P\right| \vee\left|\widetilde{z}_{+}(A)-P\right|
$$


Dans le même esprit, remarquons que $\mathbb{R}_{+}^{*} \ni A \mapsto z_{+}(a(A), A)$ est strictement croissante. En effet, si $\widetilde{A}>A$, on a

$$
z_{+}(a(A), \widetilde{A})>z_{+}(a(A), A) \quad \text { et } \quad z_{-}(a(A), \widetilde{A})<z_{-}(a(A), A)
$$

d'où l'affirmation précédente, puisque

$$
\begin{aligned}
\left|z_{-}(a(A), \widetilde{A})-P\right| \wedge\left|z_{+}(a(A), \widetilde{A})-P\right| & \leq z_{+}(a(\widetilde{A}), \widetilde{A})-P \\
& \leq\left|z_{-}(a(A), \widetilde{A})-P\right| \vee\left|z_{+}(a(A), \widetilde{A})-P\right|
\end{aligned}
$$

Il en découle par des arguments similaires à ceux présentés ci-dessus que $A(\mu, \nu)$ est également l'infimum des valeurs de $A>0$ pour lesquelles (11) admet une solution strictement positive sur $[N, M]$.

Cette dernière assertion offre un complément à la proposition 14 qui sera très important dans la section suivante et qu'il convient de signaler :

Proposition 16 La constante $A(\mu, \nu)$ peut également se réinterpréter comme l'infimum des valeurs $A>0$ pour lesquelles (11) admet une solution strictement positive sur $\left[-M_{\mu}, M_{\mu}\right]$.

Dans le cas symétrique, ceci peut se démontrer (à peine) plus facilement, en utilisant la stricte croissance de application $z$ définie avant la proposition 14 (et en jouant un peu avec la dérivée $g^{\prime}(0)$ d'une solution de (11) pour voir que si $0<A \leq A(\mu, \nu)$, elle ne peut pas être strictement positive sur $\left.\left[-M_{\mu}, M_{\mu}\right]\right)$.

Remarque 17 On n'a pas utilisé toute la richesse du théorème de comparaison de Sturm, qui envisage également les zéros suivants. Ceux-ci auraient certainement joué un rôle si au lieu de considérer la constante de Poincaré, qui correspond à l'inverse de la seconde valeur propre d'un opérateur de diffusion sous-jacent, on s'était intéressé aux valeurs propres suivantes (pour des renseignements dans cette direction, notamment sur les zéros de vecteurs propres, on peut renvoyer par exemple à la section VI.6 du livre de Courant et Hilbert [11] ou au livre de Colin de Verdière [10] dans un contexte discret de graphes). Mais nous n'aborderons pas ce sujet ici

La formulation de $A(\mu, \nu)$ donnée dans la proposition 14 admet elle-même une interprétation spectrale bien connue : $1 / A(\mu, \nu)$ est la plus petite valeur propre de l'opérateur auto-adjoint $\mathcal{L}$ : $f \mapsto-\frac{1}{V_{\nu}}\left(\frac{f^{\prime}}{\mu}\right)^{\prime}$ avec condition de Dirichlet au bord sur $\left[-M_{\mu}, M_{\mu}\right]$. Rigoureusement ceci signifie que $\mathcal{L}$ doit être compris comme la fermeture dans $\mathbb{L}^{2}(]-M_{\mu}, M_{\mu}\left[, V_{\nu} \cdot \lambda\right)$ de l'opérateur du second ordre défini comme ci-dessus sur $\mathcal{C}_{\mathrm{c}}^{2}(]-M_{\mu}, M_{\mu}[)$. De manière équivalente, on peut passer par la forme de Dirichlet correspondante : pour tout $f \in \mathbb{L}^{2}(]-M_{\mu}, M_{\mu}\left[, V_{\nu} \cdot \lambda\right)$, soit

$$
\mathcal{E}(f, f):= \begin{cases}\int_{]-M_{\mu}, M_{\mu}[}\left(f^{\prime}\right)^{2} / \mu d \lambda & , \text { si } f \text { est absolument continue de dérivée faible } f^{\prime} \\ +\infty & , \text { sinon }\end{cases}
$$

On a alors avec les conventions habituelles,

$$
\frac{1}{A(\mu, \nu)}=\inf _{f \in \mathbb{L}^{2}(]-M_{\mu}, M_{\mu}\left[, V_{\nu} \cdot \lambda\right) \backslash\{0\}} \frac{\mathcal{E}(f, f)}{\lambda\left[V_{\nu} f^{2}\right]}
$$

On peut être interpellé par la ressemblance entre cette formulation et notre définition initiale de la constante de Poincaré, qui peut se récrire

$$
\frac{1}{A(\mu, \nu)}:=\inf _{f \in H^{1}(]-M_{\mu}, M_{\mu}[) \backslash\{0\}} \frac{\int\left(f^{\prime}\right)^{2} d \nu}{\operatorname{Var}(f, \mu)}
$$


(où $H^{1}(]-M_{\mu}, M_{\mu}$ [) est l'espace de Sobolev des fonctions $f$ absolument continues sur ] $-M_{\mu}, M_{\mu}$ [ admettant une dérivée faible $f^{\prime}$ dans $\left.\mathbb{L}^{2}(]-M_{\mu}, M_{\mu}[, \lambda)\right)$ et se demander s'il n'existerait pas un passage direct entre ces égalités. Pour le décrire, notons que le dernier membre de droite vaut aussi

$$
\inf _{f \in H^{1}(]-M_{\mu}, M_{\mu}[) \backslash\{0\}: \mu[f]=0} \frac{\int\left(f^{\prime}\right)^{2} d \nu}{\mu\left[f^{2}\right]}
$$

Ensuite la procédure la plus simple pour passer de la restriction $\mu[f]=0$ à une condition de Dirichlet consiste à associer à une telle fonction $f$ l'application

$$
F:\left[-M_{\mu}, M_{\mu}\right] \ni x \mapsto \int_{-M_{\mu}}^{x} f(t) \mu(d t)
$$

vérifiant bien $F\left(-M_{\mu}\right)=0=F\left(M_{\mu}\right)$. Si $f$ appartient de plus à $H^{1}(]-M_{\mu}, M_{\mu}[)$, la fonction $F$ est dérivable, $F^{\prime}=f \mu$, et admet une dérivée seconde faible $F^{\prime \prime}$ telle que $\left(\frac{F^{\prime}}{\mu}\right)^{\prime}=f^{\prime}$. On peut donc récrire les numérateurs et dénominateurs apparaissant dans (16) en termes de $F$ :

$$
\begin{aligned}
\nu\left[\left(f^{\prime}\right)^{2}\right] & =\int\left(\left(\frac{F^{\prime}}{\mu}\right)^{\prime}\right)^{2} d \nu \\
& =\int(\mathcal{L}[F])^{2} V_{\nu} d \lambda \\
\mu\left[f^{2}\right] & =\int\left(\frac{F^{\prime}}{\mu}\right)^{2} d \mu \\
& =\int F \mathcal{L}[F] V_{\nu} d \lambda
\end{aligned}
$$

où l'on a effectué une intégration par parties en tenant compte de la condition de Dirichlet. Ainsi en désignant par $H_{0}^{1}\left(\left[-M_{\mu}, M_{\mu}\right]\right)$ l'espace de Sobolev des fonctions (nécessairement continues) sur $\left[-M_{\mu}, M_{\mu}\right]$ admettant une dérivée seconde faible dans $\mathbb{L}^{2}(]-M_{\mu}, M_{\mu}[, \lambda)$ et satisfaisant la condition de Dirichlet au bord, on a

$$
\frac{1}{A(\mu, \nu)}:=\inf _{f \in H_{0}^{1}\left(\left[-M_{\mu}, M_{\mu}\right]\right) \backslash\{0\}} \frac{\int(\mathcal{L}[F])^{2} V_{\nu} d \lambda}{\int F \mathcal{L}[F] V_{\nu} d \lambda}
$$

Cependant l'opérateur $\mathcal{L}$ étant auto-adjoint dans l'espace de Hilbert $\mathbb{L}^{2}(]-M_{\mu}, M_{\mu}\left[, V_{\nu} \cdot \lambda\right)$, on peut le décomposer spectralement (cf. par exemple le livre de Dunford et Schwartz [14]) ce qui fait apparaître dans l'expression ci-dessus que $1 / A(\mu, \nu)$ est l'infimum de son spectre, ce qui se traduit aussi par (14).

Le fait que $1 / A(\mu, \nu)$ est une véritable valeur propre de $\mathcal{L}$ et qu'il existe un vecteur propre strictement positif dans $H_{0}^{1}\left(\left[-M_{\mu}, M_{\mu}\right]\right)$ (donc sur $]-M_{\mu}, M_{\mu}$ [ et avec condition de Dirichlet au bord) découle d'une généralisation en dimension infinie du célèbre théorème de Perron-Frobenius sur les matrices irréductibles à entrées positives (pour ce point de vue, on renvoie aux ouvrages [37] de Reed et Simon ou [17] de Glimm et Jaffe). Mais l'énoncer nous aurait amené à introduire des notions un peu sophistiquées (comme celle de semi-groupe hypercontractif améliorant la positivité, voir le théorème XIII.49 de [37]) et nous avons donc préféré l'approche plus élémentaire basée sur les théorèmes de Sturm, d'autant plus que c'est la proposition 16 qui sera cruciale pour nous.

Remarque 18 L'identité

$$
\inf _{f \in H^{1}(]-M_{\mu}, M_{\mu}[) \backslash\{0\}} \frac{\int\left(f^{\prime}\right)^{2} \frac{\nu}{\mu} d \mu}{\operatorname{Var}(f, \mu)}=\inf _{f \in \mathbb{L}^{2}(]-M_{\mu}, M_{\mu}\left[, V_{\nu} \cdot \lambda\right) \backslash\{0\}} \frac{\mathcal{E}(f, f)}{\lambda\left[V_{\nu} f^{2}\right]}
$$

peut se traduire de manière probabiliste comme suit : évaluer la vitesse de convergence à l'équilibre (au sens $\mathbb{L}^{2}(\mu)$ ) pour la diffusion $X$ réfléchie dans $\left[-M_{\mu}, M_{\mu}\right]$ de prégénérateur $f \mapsto\left(\nu f^{\prime \prime}+\nu^{\prime} f^{\prime}\right) / \mu$ 
(avec donc condition de Neumann au bord, $f^{\prime}\left(-M_{\mu}\right)=0=f^{\prime}\left(M_{\mu}\right)$, ce qui est relié à la définition (15)) revient aussi à estimer la vitesse de disparition (dans $\mathbb{L}^{2}(]-M_{\mu}, M_{\mu}\left[, V_{\nu} \cdot \lambda\right)$ ) pour la diffusion $Y=\left(Y_{t}\right)_{t \geq 0}$ absorbée sur la frontière de prégénérateur $f \mapsto \nu\left(f^{\prime \prime} / \mu-\mu^{\prime} f^{\prime} /(\mu)^{2}\right)$ (ce qui correspond à (14), avec condition de Dirichlet au bord $f\left(-M_{\mu}\right)=0=f\left(M_{\mu}\right)$ ). Ceci peut être intéressant d'un point de vue pratique, car cette dernière vitesse (qui vaut aussi $\lim _{t \rightarrow+\infty} t^{-1} \ln \left(\mathbb{P}\left[Y_{t} \in\right]-M_{\mu}, M_{\mu}[]\right.$ ) si $Y_{0}$ est porté par $]-M_{\mu}, M_{\mu}[$ ) est plus facile à estimer sur un grand échantillon de trajectoires de $Y$ (par exemple issues de 0 ) que la convergence à l'équilibre pour le processus $X$. Malheureusement ce simili principe de dualité semble restreint à la dimension 1.

Enfin pour finir cette section, effectuons une dernière réduction : "les analystes" savent très bien supprimer le $\mu$ de l'équation de Sturm-Liouville par un changement de temps, quitte à y modifier $V_{\nu}$ (voir le paragraphe XI.1 de [19]). Dans notre cadre cela revient à tout transférer par le biais de la fonction de répartition $F_{\mu}$ (astuce permettant de voir toute loi sur $\mathbb{R}$ comme une image de la loi uniforme sur [0,1]). Cette opération a été utilisée par Bobkov et Götze [4], mais dans notre contexte de constante de Poincaré symétrique, il plus commode d'avoir plutôt recours à la loi uniforme sur $[-1,1]$.

Notons donc $G$ l'application

$$
\left[-M_{\mu}, M_{\mu}\right] \ni x \mapsto-1+2 \mu\left(\left[-M_{\mu}, x\right]\right) \in[-1,1]
$$

Sous nos hypothèses de régularité, il s'agit d'un $\mathcal{C}^{\infty}$-difféomorphisme et soit $G^{-1}:[-1,1] \rightarrow$ $\left[-M_{\mu}, M_{\mu}\right]$ son inverse. En effectuant le changement de variable $h=g \circ G^{-1}$, on calcule que

$$
h^{\prime}=g^{\prime} \circ G^{-1}\left(G^{-1}\right)^{\prime}=g^{\prime} \circ G^{-1} \frac{1}{G^{\prime} \circ G^{-1}}=\frac{g^{\prime}}{2 \mu} \circ G^{-1}
$$

ainsi l'équation (11) se transforme en

$$
A \frac{h^{\prime \prime}}{2}=-V h
$$

avec la fonction de classe $\mathcal{C}^{\infty}$

$$
V:[-1,1] \ni u \mapsto \frac{1}{8} \frac{V_{\nu}}{\mu}\left(G^{-1}(u)\right) \in \mathbb{R}_{+}^{*}
$$

Réciproquement les solutions de (18) se transforment en celles de (11) par la composition $g=h \circ G$. On est ainsi ramené à trouver les $A>0$ pour lesquels il existe des solutions strictement positives de (18) sur $[-1,1]$. Evidemment, le préfacteur 1/2 du laplacien laisse présager du traitement "probabiliste" qui va être donné à ce problème dans la section suivante.

Remarque 19 On trouvera de nombreuses informations très intéressantes sur ce type de question et ses généralisations (en particulier multidimensionnelles) dans le livre de Pinsky [34], dont on aurait aussi pu invoquer la méthode plus abstraite pour éviter la promenade par les théorèmes de Sturm. En en reprenant la terminologie, $A(\mu, \nu)$ apparaît, à partir de (18), comme l'infimum des $A>0$ tels que l'opérateur $\frac{1}{2} \frac{d^{2}}{d x^{2}}+\frac{V}{A}$ sur ] $-1,1[$ soit surcritique. Ainsi le théorème 2.4 p. 227 de [34] propose un encadrement d'une telle constante pour les perturbations du laplacien (en dimension supérieure à 3) par des potentiels radiaux à support compact. Néanmoins, cette situation se ramène à un problème diffusif sur $] 0,+\infty[$ et il serait certainement très instructif de comparer les bornes présentées par Pinsky avec une version "désymétrisée" des résultats qui vont suivre. Plus généralement, on peut s'interroger sur l'extension éventuelle de ces derniers à des cadres multidimensionnels (pour le traitement des inégalités de Hardy correspondantes grâce à une approche basée sur les capacités, on renvoie par exemple au livre de Maz'ja [26]). 


\section{Des fonctionnelles browniennes}

En poursuivant une approche analytique, notamment par le biais d'un changement de variable logarithmique, Bobkov et Götze [4] ont transformé (18) en une équation de Riccati. Puis en se basant sur le temps d'explosion de la solution à cette équation, ils ont abouti à un critère relatif à la constante de Hardy. Nous allons plutôt chercher à caractériser $A(\mu, \nu)$ à partir de la finitude de certaines intégrales de Feynman-Kac (puis de transformées de Laplace de fonctionnelles quadratiques du mouvement brownien), mais les deux méthodes ne sont pas sans relations. A nouveau, il s'agit de manipulations très classiques, pour diverses généralisations (multidimensionnelles, avec $V$ non-positif etc.), on renvoie par exemple aux articles [7] de Chung et Rao et [33] de Pinsky.

On s'intéresse donc à l'équation (18), où $A>0$ et $V:[-1,1] \rightarrow \mathbb{R}_{+}^{*}$ est une fonction classe $\mathcal{C}^{\infty}$. Comme dans la section précédente, il est parfois commode d'étendre $V$ sur $\mathbb{R}$ en une fonction $\mathcal{C}^{\infty}$, uniformément minorée et majorée par des constantes strictement positives (et disons globalement lipschitzienne, bien que cela ne soit pas nécessaire dans cette situation) de sorte que les solutions de (18) sont aussi définies sur $\mathbb{R}$. Notons $A(V)>0$ l'infimum des $A>0$ pour lesquels il existe une solution de $(18)$ strictement positive sur $[-1,1]$. D'après les considérations de la remarque 15 , cette constante est aussi l'unique $A$ pour lequel il existe une solution de (18) strictement positive sur ] $-1,1[$ et avec condition de Dirichlet au bord $\{-1,1\}$.

Soit $\left(B_{t}\right)_{t \geq 0}$ un mouvement brownien standard et notons

$$
\tau:=\inf \left\{t \geq 0:\left|B_{t}\right|=1\right\}
$$

son temps d'atteinte de 1 ou -1 .

Notre premier objectif est de retrouver rapidement la

Proposition 20 La constante $A(V)$ coïncide avec l'infimum des $A>0$ pour lesquels

$$
\mathbb{E}_{0}\left[\exp \left(\frac{1}{A} \int_{0}^{\tau} V\left(B_{s}\right) d s\right)\right]<+\infty
$$

(le 0 en indice de l'espérance rappelle que l'on considère un mouvement brownien issu de 0).

Comme le montrent les arguments ci-dessous, l'infimum précédent, tout comme $A(V)$, n'est pas atteint.

\section{Preuve :}

Bien que le résultat de cette proposition soit classique, donnons-en une démonstration très simple, mais typique de la dimension 1 et basée sur les discussions de la section précédente.

Commençons par considérer $A>A(V)$ et soit $h$ une solution de (18) strictement positive sur $[-1,1]$. Pour $t \geq 0$, notons

$$
M_{t}:=\exp \left(\frac{1}{A} \int_{0}^{t} V\left(B_{s}\right) d s\right) h\left(B_{s}\right)
$$

Nos hypothèses de régularité permettent d'appliquer la formule d'Itô, qui affirme qu'infinitésimalement,

$$
\begin{aligned}
d M_{t} & =M_{t} \frac{V\left(B_{t}\right)}{A} d t+\exp \left(\frac{1}{A} \int_{0}^{t} V\left(B_{s}\right) d s\right)\left(h^{\prime}\left(B_{t}\right) d B_{t}+\frac{h^{\prime \prime}\left(B_{t}\right)}{2} d t\right) \\
& =\exp \left(\frac{1}{A} \int_{0}^{t} V\left(B_{s}\right) d s\right) h^{\prime}\left(B_{t}\right) d B_{t}
\end{aligned}
$$

On en déduit que le processus $\left(M_{t}\right)_{t \geq 0}$ est une martingale. Par le théorème d'arrêt de Doob, il en va de même pour $\left(M_{t \wedge \tau}\right)_{t \geq 0}$, car manifestement $\tau$ est un temps d'arrêt. Par ailleurs, puisque $\tau$ est 
$\mathbb{P}_{0}$-p.s. fini, on a que pour $t \geq 0$ grand, $M_{t \wedge \tau}$ converge $\mathbb{P}_{0}$-p.s. vers $M_{\tau}$. Par une application du lemme de Fatou, il ressort alors que

$$
\begin{aligned}
\mathbb{E}_{0}\left[\exp \left(\frac{1}{A} \int_{0}^{\tau} V\left(B_{s}\right) d s\right)\right] & \leq \frac{1}{h(-1) \wedge h(1)} \mathbb{E}_{0}\left[h\left(B_{\tau}\right) \exp \left(\frac{1}{A} \int_{0}^{\tau} V\left(B_{s}\right) d s\right)\right] \\
& \leq \liminf _{t \rightarrow+\infty} \frac{1}{h(-1) \wedge h(1)} \mathbb{E}_{0}\left[M_{t \wedge \tau}\right] \\
& =\frac{1}{h(-1) \wedge h(1)} \mathbb{E}_{0}\left[M_{0}\right] \\
& =\frac{h(0)}{h(-1) \wedge h(1)}<+\infty
\end{aligned}
$$

Réciproquement, prenons $A \leq A(V)$. Les arguments de la section précédente montrent alors qu'il existe $0 \leq \eta<1$ et une solution $h$ de (18) strictement positive sur $[-(1-\eta), 1-\eta]$ et telle que $h(-(1-\eta))=0=h(1-\eta)$. Soit $0<\epsilon<1-\eta, h$ est donc une solution strictement positive de (18) sur $[-(1-\eta-\epsilon), 1-\eta-\epsilon]$. Notons

$$
\begin{aligned}
\tau_{\epsilon}^{-} & :=\inf \left\{t \geq 0: B_{t}=-(1-\eta-\epsilon)\right\} \\
\tau_{\epsilon}^{+} & :=\inf \left\{t \geq 0: B_{t}=1-\eta-\epsilon\right\} \\
\tau_{\epsilon} & :=\tau_{\epsilon}^{-} \wedge \tau_{\epsilon}^{+}=\inf \left\{t \geq 0:\left|B_{t}\right|=1-\eta-\epsilon\right\}
\end{aligned}
$$

D'après ce qui précède, on est assuré de

$$
\mathbb{E}_{0}\left[\exp \left(\frac{1}{A} \int_{0}^{\tau_{\epsilon}} V\left(B_{s}\right) d s\right)\right]<+\infty
$$

et on peut alors transformer l'argument de Fatou en une convergence dominée, pour voir que

$$
\begin{aligned}
h(0)= & \mathbb{E}_{0}\left[h\left(B_{\tau_{\epsilon}}\right) \exp \left(\frac{1}{A} \int_{0}^{\tau_{\epsilon}} V\left(B_{s}\right) d s\right)\right] \\
= & h(-(1-\eta-\epsilon)) \mathbb{E}_{0}\left[\exp \left(\frac{1}{A} \int_{0}^{\tau_{\epsilon}} V\left(B_{s}\right) d s\right) \mathbb{1}_{\left\{\tau_{\epsilon}^{-}<\tau_{\epsilon}^{+}\right\}}\right] \\
& +h(1-\eta-\epsilon) \mathbb{E}_{0}\left[\exp \left(\frac{1}{A} \int_{0}^{\tau_{\epsilon}} V\left(B_{s}\right) d s\right) \mathbb{1}_{\left\{\tau_{\epsilon}^{+}<\tau_{\epsilon}^{-}\right\}}\right]
\end{aligned}
$$

Dans les cas où $V$ est pair, les deux termes de la somme précédente sont égaux. Mais de manière générale, le fait que $h(0)>0$ et les convergences

$$
\lim _{\epsilon \rightarrow 0_{+}} h(-(1-\eta-\epsilon))=0=\lim _{\epsilon \rightarrow 0_{+}} h(1-\eta-\epsilon)
$$

impliquent que

$$
\lim _{\epsilon \rightarrow 0_{+}} \mathbb{E}_{0}\left[\exp \left(\frac{1}{A} \int_{0}^{\tau_{\epsilon}} V\left(B_{s}\right) d s\right) \mathbb{1}_{\left\{\tau_{\epsilon}^{-}<\tau_{\epsilon}^{+}\right\}}\right] \vee \mathbb{E}_{0}\left[\exp \left(\frac{1}{A} \int_{0}^{\tau_{\epsilon}} V\left(B_{s}\right) d s\right) \mathbb{1}_{\left\{\tau_{\epsilon}^{+}<\tau_{\epsilon}^{-}\right\}}\right]=+\infty
$$

d'où notamment

$$
\lim _{\epsilon \rightarrow 0_{+}} \mathbb{E}_{0}\left[\exp \left(\frac{1}{A} \int_{0}^{\tau_{\epsilon}} V\left(B_{s}\right) d s\right)\right]=+\infty
$$

On en déduit sans difficulté que

$$
\mathbb{E}_{0}\left[\exp \left(\frac{1}{A} \int_{0}^{\tau} V\left(B_{s}\right) d s\right)\right]=+\infty
$$


puis le résultat annoncé

Remarque 21 Si $A>A(V)$, une solution strictement positive de (18) sur [-1,1] est donnée par

$$
\widetilde{h}:[-1,1] \ni x \mapsto \mathbb{E}_{x}\left[\exp \left(\frac{1}{A} \int_{0}^{\tau} V\left(B_{s}\right) d s\right)\right] \in[1,+\infty[
$$

(en appliquant la propriété de Markov forte au temps d'arrêt $\tau_{x}:=\inf \left\{t \geq 0:\left|B_{t}\right|=|x|\right\}$, on montre facilement que le membre de droite est fini pour tout $x \in[-1,1]$ si et seulement s'il l'est pour $x=0$, point où $\widetilde{h}$ atteint son maximum, on a notamment $\widetilde{h}^{\prime}(0)=0$ si $\left.\widetilde{h}(0)<+\infty\right)$. On se base souvent sur cette observation pour montrer que si $\mathbb{E}_{0}\left[\exp \left(\frac{1}{A} \int_{0}^{\tau} V\left(B_{s}\right) d s\right)\right]<+\infty$, alors il existe bien une solution strictement positive de $(18)$ sur $[-1,1]$. En effet, le processus $\left(M_{t}\right)_{t \geq 0}$ défini par

$$
\begin{aligned}
\forall t \geq 0, \quad M_{t} & :=\mathbb{E}_{0}\left[\exp \left(\frac{1}{A} \int_{0}^{\tau} V\left(B_{s}\right) d s\right) \mid \sigma\left(B_{u}, 0 \leq u \leq t\right)\right] \\
& =\exp \left(\frac{1}{A} \int_{0}^{t \wedge \tau} V\left(B_{s}\right) d s\right) \widetilde{h}\left(X_{t \wedge \tau}\right)
\end{aligned}
$$

est manifestement dans cette situation une martingale (uniformément intégrable). Ainsi le calcul d'Itô permettra de se convaincre que $\widetilde{h}$ est une solution (évidemment strictement positive sur $[-1,1])$ de $(18)$, dès que l'on saura que $\widetilde{h}$ est suffisamment régulier : par exemple continu sur $[-1,1]$ et de classe $\mathcal{C}^{2}$ à l'intérieur. Ceci n'est pas très compliqué à obtenir, en comparant directement $\widetilde{h}(x)$ et $\widetilde{h}(x+\eta)$ pour $x \in]-1,1[$ et $|\eta|$ assez petit (de manière probabiliste, en ayant recours à des temps d'arrêt appropriés), mais est déjà plus technique que les arguments présentés ci-dessus.

Pour plus d'informations et une amorce de bibliographie sur le sujet, on renvoie à la section 6.4 du livre [8] de Chung et Williams ou à celui de Chung et Zhao [9] pour une étude plus avancée.

A partir de maintenant, nous supposerons de plus explicitement que le potentiel $V$ est pair. Ceci va permettre d'appliquer sans délais un théorème de Ray-Knight.

Plus précisément, notons par $\left(L_{t}(x)\right)_{t \in \mathbb{R}_{+}, x \in \mathbb{R}}$ la famille bi-continue des temps locaux associés au mouvement brownien standard $\left(B_{t}\right)_{t \geq 0}$. Ceux-ci peuvent être définis à partir de la formule des temps d'occupations (cf. par exemple le livre de Revuz et Yor [38]) : pour toute fonction $f$ borélienne et positive sur $\mathbb{R}$, on a $\mathbb{P}_{0}$-p.s.,

$$
\int_{0}^{t} f\left(B_{s}\right) d s=\int_{\mathbb{R}} f(x) L_{t}(x) d x
$$

Cependant, du fait que $V$ est supposée paire, on est plutôt amené à considérer $\left(l_{t}(x)\right)_{t \in \mathbb{R}_{+}, x \in \mathbb{R}_{+}}$, avec $l_{t}(0)=L_{t}(0)$ et $l_{t}(x)=L_{t}(x)+L_{t}(-x)$ pour tous $t, x \geq 0$. Il s'agit des temps locaux pour le processus de Bessel de dimension $1,\left(\left|B_{t}\right|\right)_{t \geq 0}$, et un théorème de type Ray-Knight (voir le théorème $4.1 \mathrm{du}$ livre de Yor [42] et y prendre $\gamma=1)$ affirme que la loi du processus $\left(l_{\tau}(1-x)\right)_{0 \leq x \leq 1}$ est celle d'un carré de Bessel de dimension $2,\left(W_{x}^{2}+\widetilde{W}_{x}^{2}\right)_{0 \leq x \leq 1}$, où $\left(W_{x}\right)_{x \geq 0}$ et $\left(\widetilde{W}_{x}\right)_{x \geq 0}$ sont deux mouvements browniens standards.

On en déduit notamment que $\int_{0}^{\tau} V\left(B_{s}\right) d s$ a même loi que $\int_{0}^{1} V(1-x) W_{x}^{2} d x+\int_{0}^{1} V(1-x) \widetilde{W}_{x}^{2} d x$. Ainsi par indépendance et identique distribution de ces deux dernières intégrales, on a pour tout $A>0$,

$$
\mathbb{E}_{0}\left[\exp \left(\frac{1}{A} \int_{0}^{\tau} V\left(B_{s}\right) d s\right)\right]=\left(\mathbb{E}_{0}\left[\exp \left(\frac{1}{A} \int_{0}^{1} V(1-s) W_{s}^{2} d s\right)\right]\right)^{2}
$$

d'où le 
Lemme 22 La constante $A(V)$ est aussi l'infimum des valeurs $A>0$ pour lesquelles

$$
\mathbb{E}_{0}\left[\exp \left(\frac{1}{A} \int_{0}^{1} V(1-s) B_{s}^{2} d s\right)\right]<+\infty
$$

où $\left(B_{s}\right)_{s \geq 0}$ désigne toujours un mouvement brownien standard.

Toutes les caractérisations de la constante $A(V)$ que nous avons données jusqu'à présent sont clairement des fonctionnelles croissantes de la fonction $(V(s))_{0 \leq s \leq 1}$, mais comme nous l'avons suggéré dans l'introduction, il est plus instructif de la voir plutôt comme une fonctionnelle croissante de l'application $F$ définie par

$$
\forall 0 \leq s \leq 1, \quad F(s):=\int_{0}^{s} V(u) d u
$$

Pour aller dans cette direction, commençons par donner un critère sur $A(V)$ ne faisant intervenir explicitement que des intégrales de $F$. La monotonicité est un peu moins immédiate et nous la réservons pour la section suivante.

Proposition 23 Pour $A>0$, soit $X_{A}$ le processus gaussien défini sur l'intervalle de temps [0,1] par

$$
\forall s \in[0,1], \quad X_{A}(s):=\int_{0}^{s} \exp \left(\frac{2}{A} \int_{v}^{s} F(1-u) d u\right) d B_{v}
$$

Alors $A(V)$ est l'infimum des $A>0$ pour lesquels

$$
\mathbb{E}_{0}\left[\exp \left(\frac{2}{A^{2}} \int_{0}^{1} F^{2}(1-s) X_{A}^{2}(s) d s\right)\right]<+\infty
$$

\section{Preuve :}

Comme on l'aura deviné, la première étape consiste à appliquer une intégration par partie stochastique (formule d'Itô) :

$$
\begin{aligned}
\int_{0}^{1} \frac{V}{A}(1-s) B_{s}^{2} d s & =\frac{1}{A}\left[-F(1-s) B_{s}^{2}\right]_{0}^{1}+\frac{2}{A} \int_{0}^{1} F(1-s) B_{s} d B_{s}+\frac{1}{A} \int_{0}^{1} F(1-s) d s \\
& =\frac{2}{A} \int_{0}^{1} F(1-s) B_{s} d B_{s}+\frac{1}{A} \int_{0}^{1} F(1-s) d s
\end{aligned}
$$

La dernière intégrale étant déterministe et finie, $A(V)$ est aussi l'infimum des $A>0$ pour lesquels

$$
\mathbb{E}_{0}\left[\exp \left(\frac{2}{A} \int_{0}^{1} F(1-s) B_{s} d B_{s}\right)\right]<+\infty
$$

Pour transformer cette expression, on a recours au théorème de Girsanov (voir par exemple le théorème $9.10 \mathrm{du}$ livre de Chung et Williams [8] pour se persuader que la non-bornitude de la dérive dans cette situation ne pose pas vraiment de problème) : rappelons que si $X$ est la solution de l'équation différentielle stochastique

$$
\left\{\begin{aligned}
X(0) & =0 \\
d X(s) & =d B_{s}+\frac{2}{A} F(1-s) X(s) d s
\end{aligned}\right.
$$

alors pour toute fonctionnelle mesurable et positive $K: \mathcal{C}([0,1]) \rightarrow \mathbb{R}_{+}$, on a

$$
\begin{aligned}
\mathbb{E}_{0} & {\left[K\left((X(s))_{0 \leq s \leq 1}\right)\right] } \\
\quad= & \mathbb{E}_{0}\left[K\left(\left(B_{s}\right)_{0 \leq s \leq 1}\right) \exp \left(\frac{2}{A} \int_{0}^{1} F(1-s) B_{s} d B_{s}-\frac{2}{A^{2}} \int_{0}^{1} F^{2}(1-s) B_{s}^{2} d s\right)\right]
\end{aligned}
$$


En particulier si $K$ est défini par

$$
\forall\left(f_{s}\right)_{0 \leq s \leq 1} \in \mathcal{C}([0,1]), \quad K\left(\left(f_{s}\right)_{0 \leq s \leq 1}\right):=\exp \left(\frac{2}{A^{2}} \int_{0}^{1} F^{2}(1-s) f_{s}^{2} d s\right)
$$

on obtient

$$
\mathbb{E}_{0}\left[\exp \left(\frac{2}{A} \int_{0}^{1} F(1-s) B_{s} d B_{s}\right)\right]=\mathbb{E}_{0}\left[\exp \left(\frac{2}{A^{2}} \int_{0}^{1} F^{2}(1-s) X(s)^{2} d s\right)\right]
$$

Cependant, l'e.d.s. (22) peut se résoudre explicitement par la méthode de variation des constantes et il apparait que la solution est justement le processus gaussien $X_{A}$.

Remarque 24 Mentionnons une relation entre le critère du lemme 22 et les équations de Riccati, que nous n'approfondirons pas, bien que cela permettrait une nouvelle passerelle avec l'approche de Bobkov et Götze [4].

Considérons $h_{A}$ la solution de (18) sur $\mathbb{R}_{+}$avec condition initiale $h_{A}(0)=1$ et $h_{A}^{\prime}(0)=0$ et soit $z_{A}>0$ son premier zéro. Sur $\left[0, z_{A}\left[\right.\right.$, on peut effectuer le changement de variable $v_{A}:=\left(\ln \left(h_{A}\right)\right)^{\prime}$ et constater qu'il est solution de l'équation de Riccati :

$$
v_{A}^{\prime}=-\left(v_{A}^{2}+2 \frac{V}{A}\right)
$$

Réciproquement, on récupère par intégration $h_{A}$ sur l'intervalle où $v_{A}$ n'explose pas : pour $s \geq 0$ dans ce domaine,

$$
h_{A}(s)=\exp \left(\int_{0}^{s} v_{A}(u) d u\right)
$$

Ainsi $z_{A}$ est aussi le "premier" temps d'explosion de $v_{A}$ et on peut réinterpréter $A(V)$ comme l'infimum des $A>0$ pour lesquels celui-ci intervient après l'instant 1.

Par ailleurs, intéressons-nous à l'espérance conditionnelle

$$
\mathbb{E}_{0}\left[\exp \left(\int_{1-x}^{1} \frac{V}{A}(1-s) B_{s}^{2} d s\right) \mid \sigma\left(B_{u}, 0 \leq u \leq 1-x\right)\right]
$$

Si celle-ci est finie (c'est-à-dire pour $x \geq 0$ assez proche de 0 ou pour $A>0$ assez grand), en utilisant la propriété de Markov et le caractère gaussien du mouvement brownien, on se convainc rapidement qu'elle est nécessairement de la forme $\exp \left(\varphi(x) B_{1-x}^{2} / 2+\psi(x)\right)$ où $\varphi(x), \psi(x)$ sont deux nombres positifs. On a clairement $\varphi(0)=0=\psi(0)$. Pour évaluer les deux fonctions $\varphi$ et $\psi$, calculons leurs dérivées. Soient $x \geq 0$ et $\eta>0$ petit, tels que $x+\eta \leq 1$ et

$$
\mathbb{E}_{0}\left[\exp \left(\int_{1-x-\eta}^{1} \frac{V}{A}(1-s) B_{s}^{2} d s\right)\right]<+\infty
$$

on peut alors écrire

$$
\begin{aligned}
& \mathbb{E}_{0}\left[\exp \left(\int_{1-x-\eta}^{1} \frac{V}{A}(1-s) B_{s}^{2} d s\right) \mid \sigma\left(B_{u}, 0 \leq u \leq 1-x-\eta\right)\right] \\
& \quad=\mathbb{E}_{0}\left[\exp \left(\psi(x)+\varphi(x) B_{1-x}^{2} / 2+\int_{1-x-\eta}^{1-x} \frac{V}{A}(1-s) B_{s}^{2} d s\right) \mid B_{1-x-\eta}\right]
\end{aligned}
$$

Puisque l'on va procéder à un calcul infinitésimal, on peut supposer $V$ constant sur $[1-x-\eta, 1-x]$ (plus rigoureusement le lecteur est invité à utiliser les comparaisons $V(1-x)-\left\|V^{\prime}\right\|_{\infty} \eta \leq V \leq$ 
$\left.V(1-x)+\left\|V^{\prime}\right\|_{\infty} \eta \operatorname{sur}[1-x-\eta, 1-x]\right)$ et notons $b:=\sqrt{2 V / A}$, constant sur ce petit intervalle. Il se trouve (voir par exemple le livre de Yor [42]) que par le biais d'une transformation de Girsanov (comme dans la preuve ci-dessus, qui fait alors apparaître un processus d'Ornstein-Uhlenbeck), on peut calculer explicitement l'espérance conditionnelle précédente : pour tout $y \in \mathbb{R}$,

$$
\begin{aligned}
\mathbb{E}_{0} & {\left[\exp \left(\frac{\varphi(x)}{2} B_{1-x}^{2}+\frac{b^{2}}{2} \int_{1-x-\eta}^{1-x} B_{s}^{2} d s\right) \mid B_{1-x-\eta}=y\right] } \\
& =\left(\cos (b \eta)-\frac{\varphi(x)}{b} \sin (b \eta)\right)^{-1 / 2} \exp \left(\frac{y^{2}}{2} \frac{\varphi(x) \cos (b \eta)+b \sin (b \eta)}{\cos (b \eta)-\frac{\varphi(x)}{b} \sin (b \eta)}\right)
\end{aligned}
$$

On en déduit que

$$
\begin{aligned}
\varphi(x+\eta) & =\frac{\varphi(x) \cos (b \eta)+b \sin (b \eta)}{\cos (b \eta)-\frac{\varphi(x)}{b} \sin (b \eta)} \\
\psi(x+\eta) & =\psi(x)-\frac{1}{2} \ln \left(\cos (b \eta)-\frac{\varphi(x)}{b} \sin (b \eta)\right)
\end{aligned}
$$

En effectuant un développement limité pour $\eta>0$ petit, on obtient les dérivées à droite de $\varphi$ et de $\psi$, il s'agit en fait de vraies dérivées (pour le voir remplacer $x$ par $x-\eta$ dans les expressions ci-dessus, ou plus simplement utiliser la continuité des dérivées à droite mises en évidence) et on obtient pour tout $0 \leq x \leq 1$ tel que $\mathbb{E}_{0}\left[\exp \left(\int_{1-x}^{1} \frac{V}{A}(1-s) B_{s}^{2} d s\right)\right]<+\infty$ (pour $0 \leq x<1$, ceci équivaut aussi à $\varphi(x)<1 /(1-x)$, puisque la loi de $B_{1-x}$ est une gaussienne centrée de variance $1-x)$

$$
\begin{aligned}
\varphi^{\prime}(x) & =\varphi^{2}(x)+2 \frac{V}{A}(1-x) \\
\psi^{\prime}(x) & =\frac{\varphi(x)}{2}
\end{aligned}
$$

$\mathrm{Au}$ signe près, on retrouve donc l'équation de Riccati (23), i.e. $\varphi=-v_{A}$. Or la caractérisation du lemme 22 revient bien à demander que $\psi$ n'explose pas avant l'instant 1 , ce qui équivaut aussi à la non-explosion de $\varphi$ avant 1 (concernant la condition $\varphi(x)<1 /(1-x)$ pour $0 \leq x<1$, on aura remarqué que si elle n'est pas vérifiée en un point, alors par comparaison entre $\varphi$ et la solution $[0,1[\ni y \mapsto 1 /(1-y)$ de $(24)$ avec $V \equiv 0$, on aura nécessairement que $\varphi$ explose avant 1$)$.

De telles considérations permettent en fait de retrouver le théorème de Ray-Knight que nous avons utilisé. En effet, il s'agit de voir que pour toute fonction $V:[0,1] \rightarrow \mathbb{R}_{+}$constante par morceaux, on a

$$
\mathbb{E}_{0}\left[\exp \left(\int_{0}^{t} V\left(\left|B_{s}\right|\right) d s\right)\right]=\left(\mathbb{E}_{0}\left[\exp \left(\int_{0}^{1} V(1-x) B_{x}^{2} d x\right)\right]\right)^{2}
$$

(égalité à comprendre dans $\overline{\mathbb{R}}_{+}$).

Mais en utilisant la remarque 21, le membre de gauche peut s'interpréter comme $h(0)$, où $h$ est la solution de (18) sur $[0,1]$ (avec $A=1$ ) correspondant aux conditions $h(1)=1$ et $h^{\prime}(0)=0$ (ceci s'obtient par exemple en symétrisant ce problème sur $[-1,1])$. Soit $v$ la solution du problème de Riccati associé, avec $v(0)=0=h^{\prime}(0) / h(0)$, de sorte que

$$
h(0)=h(1) \exp \left(-\int_{0}^{1} v(s) d s\right)=\exp \left(-\int_{0}^{1} v(s) d s\right)
$$

(avec la convention naturelle que $v$ vaut $-\infty$ après son temps d'explosion).

Or le fait que $V$ est constant par morceaux permet de calculer explicitement $v$ (à l'aide de la fonction arctan) ainsi que son intégrale $\int_{0}^{1} v(s) d s$. Il "reste" alors à identifier le résultat obtenu 
avec le membre de droite de (25), qui lui aussi peut se calculer explicitement dans cette situation! Ainsi d'une certaine manière le théorème de Ray-Knight précédent "encapsule" le fait que l'on sait résoudre des équations de Riccati dont le potentiel est constant par morceaux. Mais rassurons le lecteur, il existe des preuves probabilistes plus élégantes (voir par exemple le livre de Yor [42]).

Enfin précisons que le développement effectué par Bobkov et Götze [4] autour de la résolution approchée l'équation de Riccati (par la méthode de Picard) permettrait aussi de voir que $A(V)$ est une fonctionnelle croissante de $(F(s))_{0 \leq s \leq 1}$.

\section{Des résultats de comparaison et de stabilité}

Nous allons ici vérifier que $A(V)$ est bien une fonctionnelle croissante de l'application $F$ définie en (20) et qu'elle admet de bonnes propriétés de continuité par rapport à celle-ci. Revenant ensuite à $A(\mu, \nu)$, ceci nous permettra d'en avoir une caractérisation valable pour toutes probabilité $\mu$ et mesure $\nu$ (symétriques) vérifiant $\int_{0}^{M_{\mu}} 1 / \nu d \lambda<+\infty$.

Commençons par généraliser certains objets de la section précédente. Soit $\mathcal{F}$ l'ensemble des fonctions $F:] 0,1\left[\rightarrow \mathbb{R}_{+}\right.$mesurables et bornées. Si $A>0$ est donné, on définit une fonctionnelle $\mathcal{H}_{A}$ de $\mathcal{F} \times \mathcal{F}$ vers $\overline{\mathbb{R}}_{+}$, en posant pour tous $F, \widetilde{F} \in \mathcal{F}$

$$
\mathcal{H}_{A}(F, \widetilde{F}):=\mathbb{E}_{0}\left[\exp \left(\frac{2}{A^{2}} \int_{0}^{1} F^{2}(1-s)\left(\int_{0}^{s} \exp \left(\frac{2}{A} \int_{v}^{s} \widetilde{F}(1-u) d u\right) d B_{v}\right)^{2} d s\right)\right]
$$

Puis toujours pour $F, \widetilde{F} \in \mathcal{F}$, on s'intéresse à $\mathcal{A}(F, \widetilde{F})$ l'infimum des $A>0$ tels que $\mathcal{H}_{A}(F, \widetilde{F})<+\infty$. La monotonicité annoncée est alors une conséquence de l'égalité $A(V)=\mathcal{A}(F, F)$ (avec $F$ défini en $(20))$ et du

Lemme 25 Pour tout $A>0$ donné, l'application $\mathcal{H}_{A}$ est croissante $:$ pour tous $\left(F_{1}, \widetilde{F}_{1}\right),\left(F_{2}, \widetilde{F}_{2}\right) \in$ $\mathcal{F}^{2}$, les inégalités (même seulement p.p.) entre fonctions $F_{1} \leq F_{2}$ et $\widetilde{F}_{1} \leq \widetilde{F}_{2}$ impliquent $\mathcal{H}_{A}\left(F_{1}, \widetilde{F}_{1}\right) \leq$ $\mathcal{H}_{A}\left(F_{2}, \widetilde{F}_{2}\right)$. Il en découle que $\mathcal{A}$ est aussi une fonctionnelle croissante sur $\mathcal{F}^{2}$.

\section{Preuve :}

Concernant la première assertion, on peut fixer $A=1$. Pour $F \in \mathcal{F}$ et $s \in[0,1]$, notons alors

$$
Y_{F}(s):=\int_{0}^{s} \exp \left(2 \int_{v}^{s} F(1-u) d u\right) d B_{v}
$$

En revenant à la définition de l'exponentielle en tant que série, il suffit de vérifier que pour tout $n \in \mathbb{N}^{*}$,

$$
\mathcal{F}^{2} \ni(F, \widetilde{F}) \mapsto \frac{1}{n !} \mathbb{E}_{0}\left[\left(\int_{0}^{1} F^{2}(1-s) Y_{\widetilde{F}}^{2}(s) d s\right)^{n}\right]
$$

est une fonctionnelle croissante. Mais l'expression ci-dessus vaut aussi

$$
\mathbb{E}_{0}\left[\int_{0 \leq s_{1}<\cdots<s_{n} \leq 1} d s_{1} \cdots d s_{n} F^{2}\left(1-s_{n}\right) \cdots F^{2}\left(1-s_{1}\right) Y_{\widetilde{F}}^{2}\left(s_{n}\right) \cdots Y_{\widetilde{F}}^{2}\left(s_{1}\right)\right]
$$

Par une application du théorème de Fubini, on est donc ramené à montrer que pour tous $0<s_{1}<$ $\cdots<s_{n}<1$,

$$
\mathcal{F} \ni F \mapsto \mathbb{E}_{0}\left[Y_{F}^{2}\left(s_{n}\right) \cdots Y_{F}^{2}\left(s_{1}\right)\right]
$$


est croissant. Cependant, pour $F \in \mathcal{F}, Y_{F}$ est la solution de l'e.d.s.

$$
\left\{\begin{aligned}
Y_{F}(0) & =0 \\
d Y_{F}(s) & =d B_{s}+2 F(1-s) Y_{F}(s) d s \quad, \text { pour } s \in[0,1]
\end{aligned}\right.
$$

(le fait que $F$ ne soit pas défini en 0 et en 1 n'importe pas) et il en resort que pour $0<\widetilde{s}<s<1$,

$$
Y_{F}(s)=\exp \left(\int_{\widetilde{s}}^{s} 2 F(1-u) d u\right) Y_{F}(\widetilde{s})+\int_{\widetilde{s}}^{s} \exp \left(\int_{u}^{s} 2 F(1-v) d v\right) d B_{u}
$$

Ainsi pour tout $p \in \mathbb{N}^{*}$ et p.p. en $y \in \mathbb{R}$ (car pour $\widetilde{s}>0$, la loi de $Y_{F}(\widetilde{s})$ sous $\mathbb{P}_{0}$ est une gaussienne non dégénérée, donc équivalente à la mesure de Lebesgue), on a

$$
\begin{aligned}
\mathbb{E}_{0}\left[Y_{F}^{2 p}(s) \mid Y_{F}(\widetilde{s})=y\right] \\
=\sum_{0 \leq q \leq 2 p} C_{2 p}^{q} y^{2 p-q} \exp \left((2 p-q) \int_{\widetilde{s}}^{s} 2 F(1-u) d u\right) \mathbb{E}_{0}\left[\left(\int_{\widetilde{s}}^{s} \exp \left(\int_{u}^{s} 2 F(1-v) d v\right) d B_{u}\right)^{q}\right] \\
=\sum_{0 \leq q \leq p} C_{2 p}^{2 q} y^{2(p-q)} \exp \left((2 p-2 q) \int_{\widetilde{s}}^{s} 2 F(1-u) d u\right)\left(\int_{\widetilde{s}}^{s} \exp \left(\int_{u}^{s} 4 F(1-v) d v\right) d u\right)^{q} C_{2 q}^{q} \frac{q !}{2^{q}}
\end{aligned}
$$

car la variable aléatoire $\int_{\widetilde{s}}^{s} \exp \left(\int_{u}^{s} F(1-v) d v\right) d B_{u}$ est gaussienne, centrée et de variance $\int_{\tilde{s}}^{s} \exp \left(\int_{u}^{s} 4 F(1-v) d v\right) d u$. Les quantités ainsi mises en évidence sont bien croissantes en $F$.

Par récurrence sur $n \in \mathbb{N}^{*}$, on en déduit que pour tout $p \in \mathbb{N}$, et tous $0 \leq s_{1}<\cdots<s_{n} \leq 1$, l'application

$$
\mathcal{F} \ni F \mapsto \mathbb{E}_{0}\left[Y_{F}^{2 p}\left(s_{n}\right) Y_{F}^{2}\left(s_{n-1}\right) \cdots Y_{F}^{2}\left(s_{1}\right)\right]
$$

est croissante. En effet, pour se ramener à l'ordre $n-1$, il suffit d'y conditionner $Y_{F}^{2 p}\left(s_{n}\right)$ par $\sigma\left(Y_{F}\left(s_{n-1}\right), \ldots, Y_{F}\left(s_{1}\right)\right)$, ou encore par $Y_{F}\left(s_{n-1}\right)$ d'après la propriété de Markov vérifiée par $Y_{F}$, et d'utiliser la formule précédente.

La seconde affirmation du lemme est une conséquence immédiate de la première. On aura noté que pour tous $A>0$ et $F, \widetilde{F} \in \mathcal{F}, \mathcal{H}_{A}(F, \widetilde{F})=\mathcal{H}_{1}(F / A, \widetilde{F} / A)$, ce qui assure que l'ensemble des $A>0$ pour lesquels ceci est fini est un intervalle.

On aurait aussi pu essayer de montrer directement que le membre de gauche de (21) est croissant en $F \in \mathcal{F}$, mais cela ne s'est pas révélé aussi simple que cela en avait l'air a priori. Une variante de la preuve précédente conduit à des résultats de stabilité :

Lemme 26 Soient $F_{1}, \widetilde{F}_{1}, F_{2}, \widetilde{F}_{2} \in \mathcal{F}$, avec $\mathcal{A}\left(F_{1}, \widetilde{F}_{1}\right)>0$, notons

$$
\epsilon:=\int_{0}^{1}\left|F_{1}-F_{2}\right| d \lambda \quad \text { et } \quad \widetilde{\epsilon}:=\int_{0}^{1}\left|\widetilde{F}_{1}-\widetilde{F}_{2}\right| d \lambda
$$

puis considérons l'application $\mathcal{K}$ définie par

$$
\mathbb{R}_{+} \ni A \mapsto\left(\left\|F_{1}\right\|_{\infty} \vee\left\|F_{2}\right\|_{\infty}\right) \epsilon\left(\frac{A \exp \left(-\frac{2 \widetilde{\epsilon}}{A}\right)}{\mathcal{A}\left(F_{1}, \widetilde{F}_{1}\right)}-1\right)_{+}^{-2} \frac{A}{4\left\|\widetilde{F}_{1}\right\|_{\infty}}\left(\exp \left(\frac{4\left\|\widetilde{F}_{1}\right\|_{\infty}}{A}\right)-1\right) \in \overline{\mathbb{R}}_{+}
$$

(si $\left\|\widetilde{F}_{1}\right\|_{\infty}=0$, on remplace naturellement, mais contrairement à nos conventions habituelles, le produit des deux derniers facteurs par 1). Clairement il existe une unique valeur $\kappa>0$ (dépendant de toutes les quantités entrant dans la définition de $\mathcal{K})$, telle que $\mathcal{K}(\kappa)=\mathcal{A}^{2}\left(F_{1}, \widetilde{F}_{1}\right) / 8$. On a alors

$$
\mathcal{A}\left(F_{2}, \widetilde{F}_{2}\right) \leq \kappa
$$




\section{Preuve :}

En reprenant les notations de la démonstration précédente, pour $A>0$ donné, on va chercher à majorer

$$
\mathbb{E}_{0}\left[\exp \left(\frac{2}{A^{2}} \int_{0}^{1} F_{2}^{2}(1-s) Y_{\widetilde{F}_{2} / A}^{2}(s) d s\right)\right]
$$

à l'aide de $\left(F_{1}, \widetilde{F}_{1}\right)$. Commençons par la partie où interviennent les tildes. En revenant à (26), il apparaît que pour tous $0<\widetilde{s}<s<1, p \in \mathbb{N}$ et p.p. en $y \in \mathbb{R}$,

$$
\mathbb{E}_{0}\left[Y_{\widetilde{F}_{2} / A}^{2 p}(s) \mid Y_{\widetilde{F}_{2} / A}(\widetilde{s})=y\right] \leq \exp \left(\frac{4 p}{A} \int_{\widetilde{s}}^{s}\left|\widetilde{F}_{1}-\widetilde{F}_{2}\right| d \lambda\right) \mathbb{E}_{0}\left[Y_{\widetilde{F}_{1} / A}^{2 p}(s) \mid Y_{\widetilde{F}_{1} / A}(\widetilde{s})=y\right]
$$

et il en découle facilement par conditionnement et récurrence que pour tous $n \in \mathbb{N}^{*}$ et $0<s_{1}<$ $\cdots<s_{n}<1$,

$$
\mathbb{E}_{0}\left[Y_{\widetilde{F}_{2}}^{2}\left(s_{n}\right) \cdots Y_{\widetilde{F}_{2}}^{2}\left(s_{1}\right)\right] \leq \exp \left(\frac{4 n}{A} \int_{0}^{1}\left|\widetilde{F}_{1}-\widetilde{F}_{2}\right| d \lambda\right) \mathbb{E}_{0}\left[Y_{\widetilde{F}_{1}}^{2}\left(s_{n}\right) \cdots Y_{\widetilde{F}_{1}}^{2}\left(s_{1}\right)\right]
$$

En remontant à la formulation exponentielle, on obtient

$$
\mathbb{E}_{0}\left[\exp \left(\frac{2}{A^{2}} \int_{0}^{1} F_{2}^{2}(1-s) Y_{\widetilde{F}_{2}}^{2}(s) d s\right)\right] \leq \mathbb{E}_{0}\left[\exp \left(\frac{2}{A^{2}} \exp (4 \widetilde{\epsilon} / A) \int_{0}^{1} F_{2}^{2}(1-s) Y_{\widetilde{F}_{1} / A}^{2}(s) d s\right)\right]
$$

et de la même manière on vérifierait que le membre de gauche est minoré par

$$
\mathbb{E}_{0}\left[\exp \left(\frac{2}{A^{2}} \exp (-4 \widetilde{\epsilon} / A) \int_{0}^{1} F_{2}^{2}(1-s) Y_{\widetilde{F}_{1} / A}^{2}(s) d s\right)\right]
$$

Pour simplifier les notations, posons $\widetilde{A}:=A \exp (-2 \widetilde{\epsilon} / A)$ et traitons maintenant la partie sans tilde. Soit $a>0$, en utilisant la majoration

$$
F_{2}^{2} \leq(1+a) F_{1}^{2}+\left(1+\frac{1}{a}\right)\left(F_{2}-F_{1}\right)^{2}
$$

puis l'inégalité de Hölder d'exposants conjugués $1+a$ et $a /(1+a)$, on obtient

$$
\begin{aligned}
\mathbb{E}_{0}\left[\exp \left(\frac{2}{\widetilde{A}^{2}} \int_{0}^{1} F_{2}^{2}(1-s) Y_{\widetilde{F}_{1} / A}^{2}(s) d s\right)\right] \\
\leq \mathbb{E}_{0}\left[\exp \left(\frac{2}{\widetilde{A}^{2}}(1+a)^{2} \int_{0}^{1} F_{1}^{2}(1-s) Y_{\widetilde{F}_{1} / A}^{2}(s) d s\right)\right]^{1 /(1+a)} \\
\mathbb{E}_{0}\left[\exp \left(\frac{2}{\widetilde{A}^{2}}\left(1+\frac{1}{a}\right)^{2} \int_{0}^{1}\left(F_{2}-F_{1}\right)^{2}(1-s) Y_{\widetilde{F}_{1} / A}^{2}(s) d s\right)\right]^{a /(1+a)}
\end{aligned}
$$

Puisque l'on s'intéresse à la finitude de telles expressions, évaluons pour quels $a>0$ on est sûr que la dernière espérance est finie. En utilisant le résultat de comparaison du lemme précédent, on peut y remplacer $\widetilde{F}_{1}$ par sa norme infinie $l:=\left\|\widetilde{F}_{1}\right\|_{\infty}$ et considérons $Z:=\max _{0 \leq s \leq 1} Y_{l / A}^{2}(s)$. Rappelons que quitte éventuellement à agrandir l'espace de probabilité sous-jacent, il existe un mouvement brownien standard $\left(W_{s}\right)_{s \geq 0}$ tel que l'on puisse écrire

$$
\forall 0 \leq s \leq 1, \quad \int_{0}^{s} \exp \left(-\frac{2}{A} \int_{0}^{v} l d u\right) d B_{v}=W_{\int_{0}^{s}} \exp \left(-\frac{4}{A} \int_{0}^{v} l d u\right) d v
$$

(voir par exemple le livre de Revuz et Yor [38]). En revenant à l'écriture

$$
\forall 0 \leq s \leq 1, \quad Y_{l / A}(s)=\exp \left(\frac{2}{A} \int_{0}^{s} l d u\right) \int_{0}^{s} \exp \left(-\frac{2}{A} \int_{0}^{v} l d u\right) d B_{v}
$$


il apparaît que l'on peut majorer $Z$ par

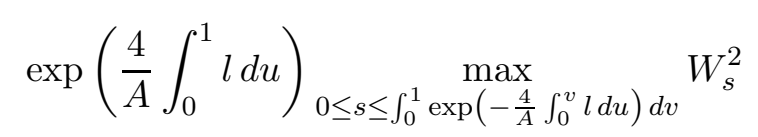

et par la propriété usuelle de changement d'échelle du mouvement brownien, ceci est égale en loi à

$$
\exp (4 l / A) \int_{0}^{1} \exp (-4 l v / A) d v\left(B_{*} \vee B^{*}\right)^{2}=\frac{A}{4 l}\left(\exp \left(\frac{4 l}{A}\right)-1\right)\left(B_{*} \vee B^{*}\right)^{2}
$$

(si $l=0$, on convient naturellement que le produit des deux premiers facteurs du membre de droite vaut 1$)$, avec

$$
B_{*}:=-\min _{0 \leq s \leq 1} B_{s} \quad \text { et } \quad B^{*}:=\max _{0 \leq s \leq 1} B_{s}
$$

et il est bien connu (voir [38]) que ces deux variables aléatoires ont même distribution que la valeur absolue d'une gaussienne centrée réduite. En récapitulant, on a donc

$$
\begin{aligned}
\mathbb{E}_{0}\left[\exp \left(\frac{2}{\widetilde{A}^{2}}\left(1+\frac{1}{a}\right)^{2} \int_{0}^{1}\left(F_{2}-F_{1}\right)^{2}(1-s) Y_{\widetilde{F}_{1} / A}^{2}(s) d s\right)\right] \\
\leq \mathbb{E}_{0}\left[\exp \left(\frac{2}{\widetilde{A}^{2}}\left(1+\frac{1}{a}\right)^{2} \int_{0}^{1}\left(F_{2}-F_{1}\right)^{2}(1-s) Z d s\right)\right] \\
\leq \mathbb{E}_{0}\left[\exp \left(\frac{4}{\widetilde{A}^{2}}\left(1+\frac{1}{a}\right)^{2}\left(\left\|F_{1}\right\|_{\infty} \vee\left\|F_{2}\right\|_{\infty}\right) \epsilon Z d s\right)\right] \\
\leq \mathbb{E}_{0}\left[\exp \left(\frac{4}{\widetilde{A}^{2}}\left(1+\frac{1}{a}\right)^{2}\left(\left\|F_{1}\right\|_{\infty} \vee\left\|F_{2}\right\|_{\infty}\right) \epsilon \frac{A}{4 l}\left(\exp \left(\frac{4 l}{A}\right)-1\right)\left(B_{*} \vee B^{*}\right)^{2}\right)\right] \\
\leq \mathbb{E}_{0}\left[\exp \left(\frac{4}{\widetilde{A}^{2}}\left(1+\frac{1}{a}\right)^{2}\left(\left\|F_{1}\right\|_{\infty} \vee\left\|F_{2}\right\|_{\infty}\right) \epsilon \frac{A}{4 l}\left(\exp \left(\frac{4 l}{A}\right)-1\right)\left(B^{*}\right)^{2}\right)\right] \\
+\mathbb{E}_{0}\left[\exp \left(\frac{4}{\widetilde{A}^{2}}\left(1+\frac{1}{a}\right)^{2}\left(\left\|F_{1}\right\|_{\infty} \vee\left\|F_{2}\right\|_{\infty}\right) \epsilon \frac{A}{4 l}\left(\exp \left(\frac{4 l}{A}\right)-1\right) B_{*}^{2}\right)\right] \\
=2 \mathbb{E}_{0}\left[\exp \left(\frac{4}{\widetilde{A}^{2}}\left(1+\frac{1}{a}\right)^{2}\left(\left\|F_{1}\right\|_{\infty} \vee\left\|F_{2}\right\|_{\infty}\right) \epsilon \frac{A}{4 l}\left(\exp \left(\frac{4 l}{A}\right)-1\right) B_{1}^{2}\right)\right]
\end{aligned}
$$

et cette dernière expression est clairement finie si et seulement si

$$
\frac{4}{\widetilde{A}^{2}}\left(1+\frac{1}{a}\right)^{2} \frac{A}{4 l}\left(\exp \left(\frac{4 l}{A}\right)-1\right)\left(\left\|F_{1}\right\|_{\infty} \vee\left\|F_{2}\right\|_{\infty}\right) \epsilon<1 / 2
$$

Par ailleurs, si $\widetilde{A} /(1+a)>\mathcal{A}\left(F_{1}, \widetilde{F}_{1}\right)$, on a, puisque $\widetilde{A} /(1+a) \leq A$,

$$
\begin{aligned}
& \mathbb{E}_{0} {\left[\exp \left(\frac{2}{\widetilde{A}^{2}}(1+a)^{2} \int_{0}^{1} F_{1}^{2}(1-s) Y_{\widetilde{F}_{1} / A}^{2}(s) d s\right)\right] } \\
& \leq \mathbb{E}_{0}\left[\exp \left(\frac{2}{\widetilde{A}^{2}}(1+a)^{2} \int_{0}^{1} F_{1}^{2}(1-s) Y_{(1+a) \widetilde{F}_{1} / \widetilde{A}^{2}}^{2}(s) d s\right)\right] \\
&<+\infty
\end{aligned}
$$

Ainsi si les inégalités $(28)$ et $\widetilde{A} /(1+a)>\mathcal{A}\left(F_{1}, \widetilde{F}_{1}\right)$ sont conjointement réalisées, on peut conclure que $\mathcal{A}\left(F_{2}, \widetilde{F}_{2}\right) \leq A$. Si $A>0$ est tel que $\widetilde{A}>\mathcal{A}\left(F_{1}, \widetilde{F}_{1}\right)$, on peut alors faire tendre $a$ vers sa valeur critique $\widetilde{A} / \mathcal{A}\left(F_{1}, \widetilde{F}_{1}\right)-1$ pour la deuxième inégalité et se retrouver avec la condition 
$\mathcal{K}(A)<\mathcal{A}^{2}\left(F_{1}, \widetilde{F}_{1}\right) / 8$. Le lemme découle ensuite aisément de la décroissance et de la continuité de l'application $\mathcal{K}$ sur le domaine où elle est finie.

Dans le lemme précédent est apparue la condition $\mathcal{A}(F, \widetilde{F})>0$ et on peut se demander pour quels $F, \widetilde{F} \in \mathcal{F}$ elle est satisfaite :

Lemme 27 Pour tous $F, \widetilde{F} \in \mathcal{F}, \mathcal{A}(F, \widetilde{F})=0$ équivaut à $F=0$ p.p. Plus précisément, pour tout $s \in] 0,1[$, on $a$

$$
\mathcal{A}(F, \tilde{F}) \geq 4 \sqrt{s / \pi \int_{0}^{1-s} F^{2}(1-u) d u}
$$

Si de plus $F$ est croissant, on a $\mathcal{A}(F, \widetilde{F}) \geq(4 / \pi) \sup _{0<s<1} F(1-s) s$.

Si $\widetilde{F}=F$, cette dernière borne est moins bonne que la première inégalité de (2), qu'il sera possible de récrire sous la forme

$$
\mathcal{A}(F, F) \geq 2 \sup _{0<s<1} F(1-s) s
$$

pour toute fonction $F \in \mathcal{F}$ croissante, mais le lemme précédent n'est qu'un résultat intermédiaire.

\section{Preuve :}

D'après le lemme 25 , on a pour $A>0$ et $F, \widetilde{F} \in \mathcal{F}$,

$$
\begin{aligned}
\mathcal{H}_{A}(F, \widetilde{F}) & \geq \mathcal{H}_{A}(F, 0) \\
& =\mathbb{E}_{0}\left[\exp \left(\frac{2}{A^{2}} \int_{0}^{1} F^{2}(1-s) B_{s}^{2} d s\right)\right]
\end{aligned}
$$

On est ainsi ramené à la situation du lemme 22 , avec $2 / A^{2}$ à la place de $1 / A$ et $F^{2}$ à la place de $V$. En reprenant alors la preuve de la proposition 23 , on obtient que $\mathcal{A}(F, 0)$ est aussi l'infimum des $A>0$ tels que

$$
\mathbb{E}_{0}\left[\exp \left(\frac{8}{A^{4}} \int_{0}^{1} G^{2}(1-s) Y_{4 G / A^{2}}^{2}(s) d s\right)\right]<+\infty
$$

avec la notation introduite dans la démonstration du lemme 25 et

$$
\forall s \in] 0,1\left[, \quad G(s):=\int_{0}^{s} F^{2}(u) d u\right.
$$

Ceci revient à dire que

$$
\mathcal{A}(F, 0)=2 \sqrt{\mathcal{A}(G, G)} \geq 2 \sqrt{\mathcal{A}(G, 0)}
$$

Ainsi si $\mathcal{A}(F, \widetilde{F})=0$, on a également $\mathcal{A}(G, 0)=0$. L'avantage est que $G$ est une fonction croissante, d'où pour tout $s \in] 0,1[$,

$$
\mathcal{H}_{A}(G, 0) \geq \mathbb{E}_{0}\left[\exp \left(\frac{2}{A^{2}} G^{2}(1-s) \int_{0}^{s} B_{u}^{2} d u\right)\right]
$$

or comme on l'a déjà vu dans la remarque 24 , le terme de droite se calcule explicitement et on voit qu'il devient infini dès que $A \leq 4 G(1-s) s / \pi$. On ne peut donc avoir $\mathcal{A}(F, \widetilde{F})=0$ que si $G(1-)=0$, c'est-à-dire si $F=0$ p.p. et la réciproque est triviale. La première minoration donnée se déduit immédiatement des considérations précédentes. La seconde découle du fait que si l'on sait a priori que $F$ est croissant, il est inutile de recourir à $G$ et on peut appliquer directement les arguments ci-dessus. 
Les arguments de la fin de la preuve du lemme 26 permettent aussi de voir que pour tous $F, \widetilde{F} \in \mathcal{F}$, $\mathcal{A}(F, \widetilde{F})<+\infty$ et un peu plus précisément que

$$
\mathcal{A}(F, \widetilde{F}) \leq \inf \left\{A>0: \frac{2\|F\|_{\infty}^{2}}{A^{2}} \frac{A}{4\|\widetilde{F}\|_{\infty}}\left(\exp \left(\frac{4\|\widetilde{F}\|_{\infty}}{A}\right)-1\right)<\frac{1}{2}\right\}
$$

(quand $\widetilde{F}=F$, le terme de droite est proportionnel à $\|F\|_{\infty}$ et ce résultat est très grossier en comparaison de la seconde inégalité de (2)).

En particulier, si l'on dispose d'une famille $\left(F_{n}\right)_{n \in \mathbb{N}}$ de fonctions de $\mathcal{F}$ uniformément bornées et convergentes dans $\mathbb{L}^{1}(] 0,1[, \lambda)$ vers $F \in \mathcal{F}$ différent de 0 dans cet espace, alors

$$
\lim _{n \rightarrow \infty} \mathcal{A}\left(F_{n}\right)=\mathcal{A}(F)
$$

où l'on désignera désormais $\mathcal{A}(F):=\mathcal{A}(F, F)$ pour tout $F \in \mathcal{F}$. En effet, en se basant sur le lemme 27 et sur (29), il apparaît qu'il existe $0<\eta \leq 1$ tel que pour tout $n$ assez grand,

$$
\eta \leq \mathcal{A}\left(F_{n}\right) \leq \eta^{-1} \text { et } \eta \leq \mathcal{A}(F) \leq \eta^{-1}
$$

En utilisant alors le lemme 26 (avec pour $n$ assez grand les assignations $F_{1}=\widetilde{F}_{1} \hookleftarrow F_{n}, F_{2}=$ $\widetilde{F}_{2} \hookleftarrow F$ puis $F_{1}=\widetilde{F}_{1} \hookleftarrow F, F_{2}=\widetilde{F}_{2} \hookleftarrow F_{n}$ ), on obtient facilement le résultat voulu. Ceci découle de l'observation suivante : notons $\kappa\left(\epsilon, \epsilon^{\prime}, \mathcal{A}\left(F_{1}, \widetilde{F}_{1}\right),\left\|F_{1}\right\|_{\infty},\left\|\widetilde{F}_{1}\right\|_{\infty},\left\|F_{2}\right\|_{\infty}\right)$ la constante $\kappa$ apparaissant dans ce lemme 26, un calcul immédiat permet de voir que

$$
\lim _{\epsilon, \epsilon^{\prime} \rightarrow 0_{+}} \kappa\left(\epsilon, \epsilon^{\prime}, a_{1}, l_{1}, \widetilde{l}_{1}, l_{2}\right)=a_{1}
$$

uniformément en $a_{1}$ dans un compact de $\mathbb{R}_{+}^{*}$ et quand $l_{1}, \widetilde{l}_{1}$ et $l_{2}$ appartiennent eux à un compact de $\mathbb{R}_{+}$.

Il est temps de revenir à une probabilité $\mu$ et à une mesure $\nu$ sympathiques, comme à la fin de la section 2, où il leur a été associé un potentiel $V$ par la formule (19). L'application $F$ correspondante est directement liée, à un changement affine près, à la restriction à ]1/2,1[ de la fonction $\widetilde{b}_{\mu, \nu} / 4$ évoquée dans l'introduction. En effet, on a par un changement de variable évident, pour tout $s \in] 0,1[$,

$$
\begin{aligned}
F(s) & =\int_{0}^{s} V d \lambda \\
& =\frac{1}{8} \int_{0}^{s} V_{\nu}\left(G_{\mu}^{-1}(u)\right) 2\left(G_{\mu}^{-1}\right)^{\prime}(u) d u \\
& =\frac{1}{4} \int_{0}^{G_{\mu}^{-1}(s)} \frac{1}{\nu} d \lambda \\
& =\frac{1}{4} \widetilde{b}_{\mu, \nu}\left(\frac{s+1}{2}\right)
\end{aligned}
$$

où l'on convient désormais de noter $G_{\mu}$ l'application considérée en (17). Cette dernière peut être définie pour toute probabilité $\mu$ et soit $G_{\mu}^{-1}$ son inverse généralisé :

$$
\begin{aligned}
\forall u \in]-1,1\left[, \quad G_{\mu}^{-1}(u)\right. & :=\inf \left\{x \in \mathbb{R}: G_{\mu}(x)>u\right\} \\
& =\inf \{x \in \mathbb{R}: \mu(]-\infty, x])>(1+u) / 2\}
\end{aligned}
$$


Par analogie avec la formule précédente, on posera pour toutes probabilité $\mu$ et mesure $\nu$ (disons symétriques sur $\mathbb{R}$ ),

$$
\forall 0<s<1, \quad F_{\mu, \nu}(s):=\frac{1}{4} \widetilde{b}_{\mu, \nu}\left(\frac{s+1}{2}\right)=\frac{1}{4} \int_{0}^{G_{\mu}^{-1}(s)} \frac{1}{\nu} d \lambda
$$

Remarquons que cette fonction est effectivement positive, grâce à l'inégalité stricte entrant dans la définition (30) (on a en effet $G^{-1}(0) \geq 0$ et même $G^{-1}(0)>0$ si 0 admet un voisinage de probabilité nulle pour $\mu$ ). Cependant, elle n'appartient à $\mathcal{F}$ que si $\int_{0}^{M_{\mu}} 1 / \nu d \lambda<+\infty$. On pourrait bien sûr retirer l'exigence de bornitude dans la définition de $\mathcal{F}$, mais certains des arguments précédents seraient mis en défaut (du fait des normes infinies apparues dans le lemme 26) et d'ailleurs le résultat suivant est faux dans cette généralité (voir le contre-exemple qui suit la proposition 29).

Proposition 28 Soient une probabilité $\mu$ et une mesure $\nu$ symétriques sur $\mathbb{R}$ telles que $M_{\mu}<+\infty$ et $\int_{0}^{M_{\mu}} 1 / \nu d \lambda<+\infty$. On a alors

$$
A(\mu, \nu)=\mathcal{A}\left(F_{\mu, \nu}\right)
$$

\section{Preuve :}

Commençons par considérer les situations où $F_{\mu, \nu}=0$ p.p. Il y a deux possibilités pour cela : soit $G_{\mu}^{-1}(1-)=0$, i.e. $\mu=\delta_{0}$, soit la densité de Radon-Nikodym $\nu$ vaut $+\infty$ p.p. sur $] 0, M_{\mu}$ [ (voir la remarque 7 ), et alors on a bien $A(\mu, \nu)=0=\mathcal{A}\left(F_{\mu, \nu}\right)$. On écarte ces deux cas pathologiques dans la discussion suivante.

Si $\mu$ et $\nu$ sont respectivement "régulières" aux sens de la fin de la section 2 , cette relation est vraie d'après la section précédente.

Ensuite si $\mu$ vérifie seulement $0<M_{\mu}<+\infty$, mais $\nu$ reste régulière (i.e. $V_{\nu}$ est $\mathcal{C}^{\infty}$ et strictement positif sur $\left.\left[0, M_{\mu}\right]\right)$, soit $\left(\mu_{n}\right)_{n \in \mathbb{N}}$ une suite de probabilités convergent adéquatement vers $\mu$. D'après les lemmes 13 et 26, pour se convaincre de l'égalité voulue il suffit de voir que pour $n$ grand, $F_{\mu_{n}, \nu}$ converge dans $\mathbb{L}^{1}(] 0,1[, \lambda)$ vers $F_{\mu, \nu}$ tout en restant borné dans $\mathbb{L}^{\infty}(] 0,1[, \lambda)$. Cette dernière bornitude étant claire, évaluons

$$
\begin{aligned}
\int_{0}^{1}\left|F_{\mu_{n}, \nu}-F_{\mu, \nu}\right| d \lambda & =\int_{0}^{1}\left|\int_{G_{\mu_{n}}^{-1}(s)}^{G_{\mu}^{-1}(s)} V_{\nu} d \lambda\right| \lambda(d s) \\
& \leq\left\|V_{\nu}\right\|_{\infty} \int_{0}^{1}\left|G_{\mu_{n}}^{-1}-G_{\mu}^{-1}\right| d \lambda
\end{aligned}
$$

expression qui converge vers 0 d'après le lemme 12.

Enfin prenons $\mu$ et $\nu$ comme dans la proposition ci-dessus (et avec $F_{\mu, \nu}$ non p.p. nulle) et soit $\left(\nu_{n}\right)_{n \in \mathbb{N}}$ une suite de mesures convergent convenablement vers $\nu$. A nouveau il suffit de voir que pour $n$ grand, $F_{\mu, \nu_{n}}$ converge dans $\mathbb{L}^{1}(] 0,1[, \lambda)$ vers $F_{\mu, \nu}$ tout en restant borné dans $\mathbb{L}^{\infty}(] 0,1[, \lambda)$. Cette dernière propriété provient de l'hypothèse $\int_{0}^{M_{\mu}} 1 / \nu d \lambda<+\infty$ et pour obtenir la convergence $\mathbb{L}^{1}$, on écrit

$$
\begin{aligned}
\int_{0}^{1}\left|F_{\mu, \nu_{n}}-F_{\mu, \nu}\right| d \lambda & \leq \int\left(\int_{0}^{G_{\mu}^{-1}(s)}\left|V_{\nu_{n}}-V_{\nu}\right| d \lambda\right) \lambda(d s) \\
& \leq \int_{0}^{1}\left|V_{\nu_{n}}-V_{\nu}\right| d \lambda
\end{aligned}
$$

d'où le résultat annoncé.

Considérons maintenant une probabilité $\mu$ et une mesure $\nu$ quelconques (symétriques). On a vu avant le lemme 10 comment les approcher par des couples $\left(\mu_{\eta}, \nu_{\eta}\right)$, avec $0<\eta<M_{\mu}$, vérifiant les 
conditions de la proposition 28. Reparamétrons cette famille par $0<\eta<1$ en prenant pour $\mu_{\eta}$ la probabilité symétrique telle que $G_{\mu_{\eta}}^{-1}(\cdot):=G_{\mu}^{-1}((1-\eta) \wedge \cdot)$ sur $] 0,1\left[\right.$, et pour $\nu_{\eta}$ la restriction de $\nu$ à ] $-G_{\mu}^{-1}(1-\eta), G_{\mu}^{-1}(1-\eta)\left[\right.$. Pour tout $0<\eta<1$, on a donc aussi $F_{\mu_{\eta}, \nu_{\eta}}(\cdot)=F_{\mu, \nu}((1-\eta) \wedge \cdot)$, ainsi la famille $\left(F_{\mu_{\eta}, \nu_{\eta}}\right)_{0<\eta<1}$ est décroissante et on a $\lim _{\eta \rightarrow 0_{+}} F_{\mu_{\eta}, \nu_{\eta}}=F_{\mu, \nu}$ au sens de la convergence simple. La convergence (7), le lemme 10 et la proposition 28 impliquent alors la

Proposition 29 Pour tous $\mu, \nu$ comme ci-dessus,

$$
A(\mu, \nu)=\lim _{\eta \rightarrow 0_{+}} \mathcal{A}\left(F_{\mu_{\eta}, \nu_{\eta}}\right)
$$

De plus si $\int_{0}^{M_{\mu}} 1 / \nu d \lambda<+\infty$, alors $F_{\mu, \nu} \in \mathcal{F}$ et $A(\mu, \nu)=\mathcal{A}\left(F_{\mu, \nu}\right)$.

Cette dernière assertion résulte du fait que dans ce cas la convergence $\lim _{\eta \rightarrow 0_{+}} F_{\mu_{\eta}, \nu_{\eta}}=F_{\mu, \nu}$ a lieu dans $\mathbb{L}^{1}(] 0,1[, \lambda)$, mais elle aurait aussi pu se montrer directement dans la preuve de la proposition 28 (sans la restriction $M_{\mu}<+\infty$ ).

Par contre, en étendant naturellement pour $A>0, \mathcal{H}_{A}$ sur $\overline{\mathcal{F}}^{2}$, avec $\overline{\mathcal{F}}$ l'ensemble des fonctions positives et mesurables sur $] 0,1\left[\right.$, puis $\mathcal{A}$ sur $\overline{\mathcal{F}}$, on n'a pas en général $A(\mu, \nu)=\mathcal{A}\left(F_{\mu, \nu}\right)$. Pour s'en convaincre, anticipons un peu sur la preuve de (2) et considérons $\mu$ la probabilité uniforme sur ] $-1,1\left[\right.$ et $\nu$ la mesure symétrique telle que $F_{\mu, \nu}(s)=F(s):=(1-s)^{-1}$ pour tout $\left.s \in\right] 0,1[$, de sorte que

$$
\begin{aligned}
B(\mu, \nu) & =\sup _{0<u<1} b_{\mu, \nu}(u) \\
& =\sup _{1 / 2<u<1} b_{\mu, \nu}\left(F_{\mu}^{-1}(u)\right) \\
& =\sup _{1 / 2<u<1}(1-u) \widetilde{b}_{\mu, \nu}(u) \\
& =4 \sup _{1 / 2<u<1}(1-u) F_{\mu, \nu}(2 u-1) \\
& =2
\end{aligned}
$$

(pour le second membre de droite, rappelons que $F_{\mu}^{-1}$ est l'inverse de la fonction de répartition de $\mu$ ), et donc $A(\mu, \nu) \leq 8$. Cependant pour cette fonction $F$ très particulière, on a $\mathcal{A}(F)=+\infty$, car pour tout $A>0, \mathcal{H}_{A}(F, F)$ vaut $+\infty$. En effet, on a même pour tout $A>0$,

$$
\mathbb{E}_{0}\left[\int_{0}^{1} F^{2}(1-s)\left(\int_{0}^{s} \exp \left(\frac{2}{A} \int_{v}^{s} F(1-u) d u\right) d B_{v}\right)^{2} d s\right]=+\infty
$$

Pour le voir calculons le membre de gauche, qui vaut

$$
\begin{aligned}
& \int_{0}^{1} F^{2}(1-s) \mathbb{E}_{0}\left[\left(\int_{0}^{s} \exp \left(\frac{2}{A} \int_{v}^{s} F(1-u) d u\right) d B_{v}\right)^{2}\right] d s \\
& =\int_{0}^{1} F^{2}(1-s)\left[\int_{0}^{s} \exp \left(\frac{4}{A} \int_{v}^{s} F(1-u) d u\right) d v\right] d s \\
& =\int_{0}^{1} \frac{1}{s^{2}} \int_{0}^{s}\left(\frac{s}{v}\right)^{\frac{4}{A}} d v d s
\end{aligned}
$$

Pour se convaincre que ceci est infini, on distingue deux cas : si $A \leq 4$, pour tout $s>0$, $\int_{0}^{s} v^{-A / 4} d v=+\infty$ et c'est terminé, sinon pour $A>4$,

$$
\begin{aligned}
\int_{0}^{1} \frac{1}{s^{2}} s^{\frac{4}{A}} \int_{0}^{s} v^{-\frac{4}{A}} d v d s & =\left(1-\frac{4}{A}\right)^{-1} \int_{0}^{1} \frac{1}{s^{2}} s d s \\
& =+\infty
\end{aligned}
$$


La proposition 29 permet déjà de se convaincre de la validité de l'une des assertions importantes de l'introduction, selon laquelle $A(\mu, \nu)$ est une fonctionnelle croissante de $\widetilde{b}_{\mu, \nu}$. En effet, soient $(\mu, \nu)$ et $\left(\mu^{\prime}, \nu^{\prime}\right)$ deux couples formés d'une probabilité et d'une mesure symétriques. Si $\widetilde{b}_{\mu, \nu} \geq \widetilde{b}_{\mu^{\prime}, \nu^{\prime}}$, on déduit que $F_{\mu, \nu} \geq F_{\mu^{\prime}, \nu^{\prime}}$ puis que pour tout $0<\eta<1, F_{\mu_{\eta}, \nu_{\eta}} \geq F_{\mu_{\eta}^{\prime}, \nu_{\eta}^{\prime}}$. Or d'après le lemme 25 et la proposition 28, on a alors $\mathcal{A}\left(F_{\mu_{\eta}, \nu_{\eta}}\right) \geq \mathcal{A}\left(F_{\mu_{\eta}^{\prime}, \nu_{\eta}^{\prime}}\right)$ et il suffit ensuite de passer à la limite $\eta \rightarrow 0_{+}$ pour obtenir $A(\mu, \nu) \geq A\left(\mu^{\prime}, \nu^{\prime}\right)$. Cette observation très simple est la base des développements des deux sections suivantes.

Remarque 30 Le fait de pouvoir toujours se ramener sur $[-1,1]$ grâce aux fonctions $G_{\mu}$ est très utile, notamment pour comparer des constantes de Poincaré de diffusions (symétriques par rapport à 0) a priori définies sur des segments différents de $\mathbb{R}$.

Cependant, on peut se demander s'il ne serait pas possible de récrire l'expression $\mathcal{H}_{A}\left(F_{\mu, \nu}, F_{\mu, \nu}\right)$, pour $A>0$, directement en termes de la probabilité $\mu$ et de la mesure $\nu$ sur leur espace d'état "naturel", i.e. $\left[-M_{\mu}, M_{\mu}\right]$. Ainsi si $\mu$ est diffuse, on a

$$
\begin{aligned}
\mathcal{H}_{A}\left(F_{\mu, \nu}, F_{\mu, \nu}\right)= & \mathbb{E}_{0}\left[\operatorname { e x p } \left\{\frac{4}{A^{2}} \int_{0}^{M_{\mu}} R^{2}(s) \exp \left(\frac{8}{A} \int_{s}^{M_{\mu}} R(u) \mu(d u)\right)\right.\right. \\
& \left.\left.W_{2 \int_{s}^{2}}^{2} \exp \left(-\frac{8}{A} \int_{v}^{M_{\mu}} R(u) \mu(d u)\right) \mu(d v) \mu(d s)\right\}\right]
\end{aligned}
$$

avec

$$
\forall u \in\left[0, M_{\mu}\left[, \quad R(u):=\frac{1}{4} \int_{0}^{u} \frac{1}{\nu} d \lambda\right.\right.
$$

et où $\left(W_{t}\right)_{t \geq 0}$ est un mouvement brownien standard. Pour s'en convaincre, il suffit de partir de l'expression alternative de $\mathcal{H}_{A}$ entrevue dans la preuve du lemme 26 (à une inversion du temps près),

$$
\begin{aligned}
& \mathcal{H}_{A}\left(F_{\mu, \nu}, F_{\mu, \nu}\right) \\
& \quad=\mathbb{E}_{0}\left[\exp \left\{\frac{2}{A^{2}} \int_{0}^{1} F_{\mu, \nu}^{2}(1-s) \exp \left(\frac{4}{A} \int_{0}^{s} F_{\mu, \nu}(1-u) d u\right) W_{\int_{1}^{s} \exp \left(-\frac{4}{A} \int_{0}^{v} F_{\mu, \nu}(1-u) d u\right) d v}^{2} d s\right\}\right]
\end{aligned}
$$

et d'utiliser de manière répétitive le changement de variable affirmant que pour tout $0<s<1$ et toute application mesurable et positive $h:\left[0, M_{\mu}\right] \rightarrow \mathbb{R}_{+}$, on a

$$
\int_{0}^{s} h\left(G_{\mu}^{-1}(1-u)\right) d u=2 \int_{G_{\mu}^{-1}(1-s)}^{M_{\mu}} h(u) \mu(d u)
$$

Par contre, cette dernière formule n'est plus nécessairement valable si $\mu$ contient des masses de Dirac (par exemple pour $h=\mathbb{1}$, dès que $1-s$ n'appartient pas à l'image de $G_{\mu}$ ), il n'est donc pas clair en général (même quand $F_{\mu, \nu}\left(M_{\mu}\right)<+\infty$ ) que $A(\mu, \nu)$ soit l'infimum de l'ensemble des $A>0$ tels que le membre de droite de (31) soit fini; cette formulation n'est pas très robuste, contrairement à celles décrites précédemment.

\section{Un calcul asymptotique}

Nous allons ici montrer la proposition 2 à l'aide des comparaisons permises par la section précédente.

Plus précisément, pour $0 \leq a<1$ fixé, soit $F^{(a)}$ l'application donnée par

$$
\left.F^{(a)}:\right] 0,1\left[\ni s \quad \mapsto \quad \mathbb{1}_{[a, 1[}(s) \frac{1}{1-s}\right.
$$


puis pour $0<\eta<1-a$, on considère la fonction aussi définie sur $] 0,1\left[\operatorname{par} F^{(a, \eta)}(\cdot):=F^{(a)}((1-\right.$ $\eta) \wedge \cdot)$.

Notre principal objectif ici est de prouver la

Proposition 31 Pour tout $0 \leq a<1$ fixé, on a

$$
\lim _{\eta \rightarrow 0_{+}} \mathcal{A}\left(F^{(a, \eta)}\right)=8
$$

Cette estimée implique essentiellement la proposition 2, mais pour le voir nous aurons besoin des deux résultats techniques suivants, où l'on reprend les notations et hypothèses de l'introduction.

Lemme 32 On a pour toutes probabilité et mesure symétriques $\mu$ et $\nu$,

$$
B(\mu, \nu)=\sup _{1 / 2 \leq u<1}(1-u) \widetilde{b}_{\mu, \nu}(u)
$$

Ceci n'aurait pas été juste si dans la définition (4) de l'inverse généralisée on avait remplacé l'inégalité stricte par une inégalité large.

\section{Preuve :}

Notons $\widetilde{B}(\mu, \nu)$ le membre de droite apparaissant dans le lemme et commençons par la borne $\widetilde{B}(\mu, \nu) \leq B(\mu, \nu)$, qui est valable quelle que soit la convention adoptée pour l'inégalité dans (4). On vérifie en effet que

$$
\left.\left.\forall 0<u<1, \quad \mu(]-\infty, F_{\mu}^{-1}(u)[) \leq u \leq \mu(]-\infty, F_{\mu}^{-1}(u)\right]\right)
$$

En tenant compte du fait que $F_{\mu}^{-1}\left(\left[1 / 2,1[) \subset \mathbb{R}_{+}\right.\right.$, il en découle que

$$
\forall 1 / 2 \leq u<1, \quad b_{\mu, \nu}\left(F_{\mu}^{-1}(u)+\right) \leq(1-u) \widetilde{b}_{\mu, \nu}(u) \leq b_{\mu, \nu}\left(F_{\mu}^{-1}(u)\right)
$$

Il reste ainsi à écrire que

$$
\begin{aligned}
\sup _{1 / 2 \leq u<1}(1-u) \widetilde{b}_{\mu, \nu}(u) & \leq \sup _{x \in F_{\mu}^{-1}([1 / 2,1[)} b_{\mu, \nu}(x) \\
& \leq \sup _{x \geq 0} b_{\mu, \nu}(x)
\end{aligned}
$$

C'est pour l'inégalité inverse $\widetilde{B}(\mu, \nu) \geq B(\mu, \nu)$ qu'il a fallu être prudent dans la définition (4). Il s'agit donc de montrer que pour tout $x \in\left[0, M_{\mu}\right]$,

$$
b_{\mu, \nu}(x) \leq \widetilde{B}(\mu, \nu)
$$

Intéressons-nous d'abord au cas où $x=M_{\mu}$.

- Si $\mu\left(\left\{M_{\mu}\right\}\right)=0$, on a $b_{\mu, \nu}\left(M_{\mu}\right)=0$ par les conventions habituelles et $M_{\mu}$ n'intervient pas vraiment dans la définition de $B(\mu, \nu)$ (i.e. $B(\mu, \nu)=\sup _{0 \leq x<M_{\mu}} b_{\mu, \nu}(x)$ ).

- Si $\mu\left(\left\{M_{\mu}\right\}\right)>0$, on peut encore écrire $b_{\mu, \nu}\left(M_{\mu}\right)=\mu\left(\left\{M_{\mu}\right\}\right) \int_{0}^{M_{\mu}} 1 / \nu d \lambda$. Soit $1-\mu\left(\left\{M_{\mu}\right\}\right)<v<1$, on a alors $F_{\mu}^{-1}(v)=M_{\mu}$, puis

$$
\begin{aligned}
\widetilde{B}(\mu, \nu) & \geq(1-v) \widetilde{b}_{\mu, \nu}(v) \\
& =(1-v) \int_{0}^{M_{\mu}} 1 / \nu d \lambda
\end{aligned}
$$

et le membre de droite converge vers $b_{\mu, \nu}\left(M_{\mu}\right)$ quand $v$ s'approche de $1-\mu\left(\left\{M_{\mu}\right\}\right)$, d'où $b_{\mu, \nu}\left(M_{\mu}\right) \leq$ $\widetilde{B}(\mu, \nu)$ dans tous les cas. 
Considérons maintenant la situation où $0 \leq x<M_{\mu}$. On a $F_{\mu}(x)<1$ ainsi $x^{\prime}:=F_{\mu}^{-1}\left(F_{\mu}(x)\right)$ est bien défini et vérifie $x^{\prime} \geq x$. De plus $\mu\left(\left[x, x^{\prime}[)=0\right.\right.$, de sorte que

$$
\begin{aligned}
b_{\mu, \nu}(x) & \leq b_{\mu, \nu}\left(x^{\prime}\right) \\
& =\int_{0}^{x^{\prime}} \frac{1}{\nu} d \lambda \mu\left(\left[x^{\prime},+\infty[)\right.\right. \\
& =\widetilde{b}_{\mu, \nu}\left(F_{\mu}(x)\right) \mu\left(\left[x^{\prime},+\infty[)\right.\right.
\end{aligned}
$$

A nouveau on distingue deux possibilités :

- Si $\mu\left(\left\{x^{\prime}\right\}\right)=0$, on a $\mu\left(\left[x^{\prime},+\infty[)=1-F_{\mu}\left(x^{\prime}\right)=1-F_{\mu}(x)\right.\right.$ et ainsi

$$
\begin{aligned}
b_{\mu, \nu}(x) & \leq\left(1-F_{\mu}(x)\right) \widetilde{b}_{\mu, \nu}\left(F_{\mu}(x)\right) \\
& \leq \widetilde{B}(\mu, \nu)
\end{aligned}
$$

- Si $\mu\left(\left\{x^{\prime}\right\}\right)>0$, comme dans le cas où $x=M_{\mu}$, on considère plutôt $F_{\mu}\left(x^{\prime}-\right)<v<F_{\mu}\left(x^{\prime}\right)$ et on fait tendre $v$ vers $F_{\mu}\left(x^{\prime}-\right)$ pour se convaincre de l'inégalité voulue.

Lemme 33 Supposons que $\mu\left(\left\{M_{\mu}\right\}\right)=0$. On a équivalence entre (3) et

$$
\lim _{u \rightarrow 1-}(1-u) \widetilde{b}_{\mu, \nu}(u)=B(\mu, \nu)
$$

\section{Preuve :}

L'hypothèse $\mu\left(\left\{M_{\mu}\right\}\right)=0$ assure que pour tout $0<u<1, M_{\mu}>F_{\mu}^{-1}(u)$, or cette quantité converge bien vers $M_{\mu}-$ en 1-. En reprenant l'inégalité de gauche de (33) obtenue dans la preuve précédente, il apparaît alors que sous la condition (3),

$$
\liminf _{u \rightarrow 1-}(1-u) \widetilde{b}_{\mu, \nu}(u) \geq B(\mu, \nu)
$$

puis la véritable convergence (34), en tenant compte du lemme 32.

Réciproquement, pour $x \in\left[0, M_{\mu}\left[\right.\right.$, soit $x^{\prime \prime}=F_{\mu}^{-1}\left(F_{\mu}(x)-\right)$, disons pour $x$ assez proche de $M_{\mu}$ de sorte que $x^{\prime \prime}$ soit strictement positif. On a alors

$$
\begin{aligned}
b(x) & \geq \int_{0}^{x^{\prime \prime}} 1 / \nu d \lambda \mu(] x^{\prime \prime},+\infty[) \\
& =\int_{0}^{x^{\prime \prime}} 1 / \nu d \lambda\left(1-F_{\mu}\left(x^{\prime \prime}\right)\right) \\
& =\int_{0}^{x^{\prime \prime}} 1 / \nu d \lambda\left(1-F_{\mu}(x)\right)
\end{aligned}
$$

$\left.\left.\operatorname{car} \mu(] x^{\prime \prime}, x\right]\right)=0$. Or quand $v>0$ tend vers $F(x)$ par valeurs strictement inférieures, $(1-v) \widetilde{b}_{\mu, \nu}(v)$ converge justement vers le dernier membre de droite, ce qui permet de conclure à l'implication (34) $\Rightarrow(3)$ sous la condition $\mu\left(\left\{M_{\mu}\right\}\right)=0$, puisqu'elle entraîne que $\lim _{x \rightarrow M_{\mu}-} F_{\mu}(x)=1$ avec $F_{\mu}(x)<1$ pour tout $0 \leq x<M_{\mu}$.

Remarque 34 Si $\mu\left(\left\{M_{\mu}\right\}\right)>0$, on obtient directement que

$$
\lim _{u \rightarrow 1-}(1-u) \widetilde{b}_{\mu, \nu}(u)= \begin{cases}+\infty & , \text { si } \int_{0}^{M_{\mu}} 1 / \nu d \lambda=+\infty \\ 0 & \text {, si } \int_{0}^{M_{\mu}} 1 / \nu d \lambda<+\infty\end{cases}
$$


Dans le premier cas, on a aussi $B(\mu, \nu)=+\infty$ et la conclusion du lemme ci-dessus reste formellement satisfaite (ainsi que celle de la proposition 2, comme on peut le voir a posteriori à partir de (2)). Par contre dans la situation où $\mu\left(\left\{M_{\mu}\right\}\right)>0$ et $\int_{0}^{M_{\mu}} 1 / \nu d \lambda<+\infty$, le lemme précédent n'est juste que dans les cas pathologiques où $\nu \equiv+\infty$ p.p. sur $\left[0, M_{\mu}[\right.$. Et mis à part pour ceux-ci, la proposition 2 sera également fausse, comme on peut s'en persuader à l'aide de comparaisons : dans cette situation on peut trouver $0<\eta<1$ tel que $F_{\mu, \nu}(\cdot) \leq B(\mu, \nu) F^{(0, \eta)}(\cdot) / 2$, or on verra dans la preuve de la proposition 31 que $\mathcal{A}\left(F^{(0, \eta)}\right)<8$ (cette valeur n'étant "atteinte" qu'asymptotiquement quand $\eta \rightarrow 0_{+}$), d'où

$$
\begin{aligned}
A(\mu, \nu) & =\mathcal{A}\left(F_{\mu, \nu}\right) \\
& \leq \frac{B(\mu, \nu)}{2} \mathcal{A}\left(F^{(0, \eta)}\right) \\
& <4 B(\mu, \nu)
\end{aligned}
$$

Ces arguments montrent plus généralement que si la fonction $F_{\mu, \nu}$ est bornée (i.e. si elle appartient à $\mathcal{F}$ ), alors

$$
A(\mu, \nu)<4 B(\mu, \nu)<+\infty
$$

et il est possible de préciser la première inégalité à partir des calculs qui vont suivre.

Admettons provisoirement la proposition 31 et déduisons-en la proposition 2. Soient $\mu$ et $\nu$ comme dans l'énoncé de cette dernière, d'après le lemme ci-dessus, on est assuré de la convergence (34) qui peut aussi se récrire

$$
\lim _{u \rightarrow 1-} 4(1-u) F_{\mu, \nu}(2 u-1)=B(\mu, \nu)
$$

ou encore

$$
\lim _{u \rightarrow 1-} 2(1-u) F_{\mu, \nu}(u)=B(\mu, \nu)
$$

Ainsi pour tout $\epsilon>0$, on peut trouver $0 \leq a_{\epsilon}<1$ tel que sur $] 0,1[$,

$$
\frac{B(\mu, \nu)}{2}(1-\epsilon) F^{\left(a_{\epsilon}\right)} \leq F_{\mu, \nu}
$$

Par ailleurs, d'après le lemme 32, on a toujours

$$
F_{\mu, \nu} \leq \frac{B(\mu, \nu)}{2} F^{(0)}
$$

Les propositions 29 et 31 permettent alors d'en déduire par comparaison que

$$
4 B(\mu, \nu)(1-\epsilon) \leq A(\mu, \nu) \leq 4 B(\mu, \nu)
$$

d'où le résultat annoncé, puisque $\epsilon>0$ peut être choisi arbitrairement petit. On aura remarqué que ces arguments assurent aussi la validité générale de la seconde inégalité de (2). On s'intéressera à la première dans la section suivante.

Pour le moment, effectuons la

\section{Preuve de la proposition 31 :}

Tout d'abord on défait à l'envers les manipulations de la preuve de la proposition 23 : la transformation de Girsanov qui y est considérée permet de se rendre compte que pour $0<\eta<1-a$, $\mathcal{A}\left(F^{(a, \eta)}\right)$ est aussi l'infimum des $A>0$ tels que

$$
\mathbb{E}_{0}\left[\exp \left(\frac{2}{A} \int_{0}^{1} F^{(a, \eta)}(1-s) B_{s} d B_{s}\right)\right]<+\infty
$$


Remarquons que

$$
2 \int_{0}^{1} F^{(a, \eta)}(1-s) B_{s} d B_{s}=\frac{2}{\eta} \int_{0}^{\eta} B_{s} d B_{s}+\int_{\eta}^{1-a} \frac{2}{s} B_{s} d B_{s}
$$

Cependant, le premier terme du membre de gauche est égal à $\left(B_{\eta}^{2}-\eta\right) / \eta$ et on calcule que le second vaut

$$
\left[\frac{B_{s}^{2}}{s}\right]_{\eta}^{1-a}-\int_{\eta}^{1-a} \frac{1}{s} d s+\int_{\eta}^{1-a} \frac{B_{s}^{2}}{s^{2}} d s
$$

Ainsi

$$
2 \int_{0}^{1} F^{(a, \eta)}(1-s) B_{s} d B_{s}=-1-\ln \left(\frac{1-a}{\eta}\right)+\frac{B_{1-a}^{2}}{1-a}+\int_{\eta}^{1-a} \frac{B_{s}^{2}}{s^{2}} d s
$$

et puisque les deux premiers termes sont déterministes, il apparaît que $\mathcal{A}\left(F^{(a, \eta)}\right)$ peut être vu comme l'infimum des $A>0$ tels que

$$
\mathbb{E}_{0}\left[\exp \left(\frac{1}{A} \int_{\eta}^{1-a} \frac{B_{s}^{2}}{s^{2}} d s+\frac{B_{1-a}^{2}}{A(1-a)}\right)\right]<+\infty
$$

(du moins formellement, il s'agit de la proposition 23 où l'on aurait pris pour tout $0<s<1$, $\left.V(s):=F^{\prime}(s)=(1-a)^{-1} \delta_{a}(s)+s^{-2} \mathbb{1}_{] a, 1-\eta[}(s)\right)$. Cependant il se trouve qu'il est possible de calculer explicitement cette quantité (puis quasiment $\mathcal{A}\left(F^{(a, \eta)}\right)$ ). Plus précisément, on verra dans le lemme qui suit cette preuve qu'en posant

$$
\forall z \in \mathbb{R}^{*}, \quad H_{a, \eta}(z):=\frac{1}{z}\left[\left(\frac{1}{4}+z\right)^{3}\left(\frac{1-a}{\eta}\right)^{2 z}-\left(\frac{1}{4}-z\right)^{3}\left(\frac{1-a}{\eta}\right)^{-2 z}\right]
$$

(cette fonction se prolonge continuement en 0 par $\left.H_{a, \eta}(0)=(6+\ln ((1-a) / \eta)) / 16\right)$, on a pour tout $z \in \mathbb{R}$

$$
\mathbb{E}_{0}\left[\exp \left(-2\left(z^{2}-1 / 16\right)\left(\int_{\eta}^{1-a} \frac{B_{s}^{2}}{s^{2}} d s+\frac{B_{1-a}^{2}}{(1-a)}\right)\right)\right]=\left(\frac{1-a}{\eta}\right)^{1 / 4} \sqrt{\frac{1}{2 H_{a, \eta}(z)}}
$$

Or les deux termes de cette égalité définissent localement où ils sont finis sur $\mathbb{C}$ des fonctions holomorphes (on aura remarqué d'une part, qu'en zéro le membre de droite se développe en une série entière admettant un rayon de convergence strictement positif et d'autre part, que $1 / H_{a, \eta}$ ne s'annule jamais, il n'y a donc pas de problème pour considérer localement une détermination holomorphe de la racine carrée). On en déduit qu'elles coïncident sur la composante connexe contenant la droite réelle de l'intérieur du domaine où elles sont finies. Notamment ce sera le cas pour $z=i y$ avec $y \in \mathbb{R}$ assez petit et si l'on pose

$$
Y(a, \eta):=\inf \left\{y>0: H_{a, \eta}(i y)=0\right\}
$$

on a

$$
\frac{1}{\mathcal{A}\left(F^{(a, \eta)}\right)}=\frac{1}{8}+2 Y(a, \eta)^{2}
$$

Il faut ensuite se convaincre que pour tout $0<a<1$,

$$
\lim _{\eta \rightarrow 0_{+}} Y(a, \eta)=0
$$


Mais pour $y \in \mathbb{R}$, la condition $H_{a, \eta}(i y)=0$ revient à demander que

$$
\left(\frac{1}{4}+i y\right)^{3}\left(\frac{1-a}{\eta}\right)^{2 i y}=\left(\frac{1}{4}-i y\right)^{3}\left(\frac{1-a}{\eta}\right)^{-2 i y}
$$

et puisque ces deux membres sont conjugués l'un de l'autre, on se ramène à trouver les $y \in \mathbb{R}_{+}$tels que

$$
\left(\frac{1}{4}+i y\right)^{3}\left(\frac{1-a}{\eta}\right)^{2 i y} \in \mathbb{R}
$$

Soit $\theta(y):=\arctan (4 y)$, de sorte que $1 / 4+i y=\sqrt{1+16 y^{2}} / 4 \exp (i \theta(y))$, la condition précédente est équivalente à $\theta(y)=-2 y \ln (a / \eta)$ modulo $\pi$. En traçant un dessin de ces fonctions, il apparaît que $Y(a, \eta)$ est le plus petit $y>0$ tel que $\arctan (4 y)=-2 y \ln (a / \eta)+\pi$. De la même manière on se persuade sans difficulté que cette quantité $Y(a, \eta)$ converge vers 0 quand $\eta$ tend vers $0_{+}$, d'où le résultat annoncé.

Ou presque, car il reste à montrer le résultat technique suivant qui repose sur l'exploitation "stochastique" de la propriété de la fonction $F:] 0,1\left[\ni s \mapsto 1 / s\right.$ d'être solution de $F^{\prime}=-F^{2}$ :

Lemme 35 Pour tous $0<\eta<1-a \leq 1$ et $z \in \mathbb{R}$, on est assuré de l'égalité (35).

\section{Preuve :}

Pour simplifier les notations, on remplacera $a$ par $1-a$, qui est désormais fixé ainsi que $\eta$ de manière à ce que $0<\eta<a \leq 1$. On a pour tout $y \in \mathbb{R}$,

$$
y \int_{\eta}^{a} \frac{B_{s}^{2}}{s^{2}} d s=y\left[-\frac{B_{s}^{2}}{s}\right]_{\eta}^{a}+2 y \int_{\eta}^{a} \frac{B_{s}}{s} d B_{s}+y \int_{\eta}^{a} \frac{d s}{s}
$$

ainsi

$$
\left(y-2 y^{2}\right) \int_{\eta}^{a} \frac{B_{s}^{2}}{s^{2}} d s=y \frac{B_{\eta}^{2}}{\eta}-y \frac{B_{a}^{2}}{a}+y \ln (a / \eta)+2 y \int_{\eta}^{a} \frac{B_{s}}{s} d B_{s}-\frac{(2 y)^{2}}{2} \int_{\eta}^{a} \frac{B_{s}^{2}}{s^{2}} d s
$$

Il en découle par une nouvelle application de la transformation de Girsanov que pour tout $y \in \mathbb{R}$,

$$
\begin{aligned}
\Psi(y) & :=\mathbb{E}_{0}\left[\exp \left(\left(y-2 y^{2}\right)\left(\int_{\eta}^{a} \frac{B_{s}^{2}}{s^{2}} d s+\frac{B_{a}^{2}}{a}\right)\right)\right] \\
& =\mathbb{E}_{0}\left[\exp \left(y \frac{B_{\eta}^{2}}{\eta}-2 y^{2} \frac{X_{a}^{2}}{a}+y \ln (a / \eta)\right)\right]
\end{aligned}
$$

avec

$$
\begin{aligned}
X_{a} & =\exp \left(\int_{\eta}^{a} \frac{2 y}{u} d u\right) B_{\eta}+\int_{\eta}^{a} \exp \left(\int_{s}^{a} \frac{2 y}{u} d u\right) d B_{s} \\
& =\left(\frac{a}{\eta}\right)^{2 y} B_{\eta}+\int_{\eta}^{a}\left(\frac{a}{s}\right)^{2 y} d B_{s}
\end{aligned}
$$

Le caractère gaussien du couple $\left(B_{\eta}, X_{a}\right)$ nous invite à calculer pour tout $x \in \mathbb{R}$, une version régulière de l'espérance conditionnelle

$$
\begin{aligned}
\Phi(x) & :=\mathbb{E}_{0}\left[\exp \left(-2 y^{2} \frac{X_{a}^{2}}{a}\right) \mid B_{\eta}=x\right] \\
& =\mathbb{E}\left[\exp \left(-\frac{2 y^{2}}{a}\left(\left(\frac{a}{\eta}\right)^{2 y} x+\sigma W\right)^{2}\right)\right]
\end{aligned}
$$


où $W$ est une variable aléatoire gaussienne centrée standard et avec

$$
\sigma:=\sqrt{\int_{\eta}^{a}\left(\frac{a}{\eta}\right)^{4 y} d s}
$$

On obtient facilement que pour tout $x \in \mathbb{R}$,

$$
\Phi(x)=\tilde{\sigma} \exp \left(\Lambda x^{2}\right)
$$

avec

$$
\begin{aligned}
\widetilde{\sigma} & :=\frac{1}{\sqrt{1+4 y^{2} \sigma^{2} / a}} \\
\Lambda & :=\frac{8 y^{4}}{a^{2}}\left(\frac{a}{\eta}\right)^{4 y} \sigma^{2} \widetilde{\sigma}^{2}-\frac{2 y^{2}}{a}\left(\frac{a}{\eta}\right)^{4 y} \\
& =-\frac{2 y^{2}}{a}\left(\frac{a}{\eta}\right)^{4 y} \frac{1}{1+4 y^{2} \sigma^{2} / a}
\end{aligned}
$$

On en déduit que pour tout $y \in \mathbb{R}$,

$$
\begin{aligned}
\Psi(y) & =\left(\frac{a}{\eta}\right)^{y} \mathbb{E}_{0}\left[\exp \left((y / \eta+\Lambda) B_{\eta}^{2}\right)\right] \tilde{\sigma} \\
& =\left(\frac{a}{\eta}\right)^{y} \tilde{\sigma} \widehat{\sigma} / \sqrt{\eta}
\end{aligned}
$$

avec

$$
\widehat{\sigma}:=\frac{1}{\sqrt{(1 / \eta-2 \Lambda-2 y / \eta)_{+}}}
$$

Evidemment il convient de se demander si cette expression est finie, c'est-à-dire si $1 / \eta-2 \Lambda-$ $2 y / \eta>0$, et pour cela il faut expliciter un peu plus les quantités précédentes. Puisque $y-2 y^{2}=$ $-2\left((y-1 / 4)^{2}-1 / 16\right)$, on s'attend à ce que le résultat final soit symétrique en $z:=y-1 / 4$ et il est commode de forcer un peu l'apparition de ce changement de variable. On obtient consécutivement $(\mathrm{du}$ moins si $y \neq 1 / 4)$,

$$
\begin{aligned}
\sigma^{2} & =a \frac{\left(\frac{a}{\eta}\right)^{4 z}-1}{4 z} \\
\frac{1}{\widetilde{\sigma}^{2}} & =\frac{1}{z}\left[\left(z+\frac{1}{4}\right)^{2}\left(\frac{a}{\eta}\right)^{4 z}-\left(z-\frac{1}{4}\right)^{2}\right] \\
\frac{1}{\widetilde{\sigma}^{2} \widehat{\sigma}^{2}} & =\frac{2}{\eta z}\left[\left(z+\frac{1}{4}\right)^{3}\left(\frac{a}{\eta}\right)^{4 z}-\left(-z+\frac{1}{4}\right)^{3}\right]
\end{aligned}
$$

où il apparaît notamment que cette quantité ne s'annule pas pour $z \in \mathbb{R}^{*}$. La formule voulue en découle immédiatement.

Remarque 36 Marc Yor vient de m'apprendre que le calcul effectué dans le lemme 35 se trouve aussi essentiellement dans un article récent de Mansuy [23] (on pourra également consulter [35, 36] ou [38] pour d'autres obtentions possibles, notamment par le biais d'équations de Sturm-Liouville, ce qui est relié à la remarque 24 ).

Par ailleurs, dans des travaux de Peccati et Yor (voir [31] ou [32]), des quantités similaires à 
celles considérées ci-dessus sont étudiées. Ainsi ces auteurs montrent par exemple qu'au sens de la convergence en loi,

$$
\lim _{\eta \rightarrow 0_{+}}(\ln (1 / \eta))^{-\frac{1}{2}} \int_{\eta}^{1} \frac{B_{s}^{2}-s}{s^{2}} d s=2 W
$$

où $W$ est une variable gaussienne centrée réduite. Ce comportement n'est pas en contradiction avec notre évaluation de $\mathcal{A}\left(F^{(1, \eta)}\right)$, et d'une certaine manière lui est même "orthogonal", puisqu'une variable normale admet des moments exponentiels de tous ordres.

\section{Deux petites remarques}

Nous allons vérifier ici deux assertions présentées dans l'introduction, l'une concernant certains cas d'égalité dans la première borne de (2) et l'autre relative au fait que l'on ne peut pas se contenter de la convergence d'une sous-suite en (3).

Mais vérifions tout d'abord la première inégalité de (2) par des arguments de comparaison. Soient donc $\mu$ une probabilité et $\nu$ une mesure, toutes deux symétriques $\operatorname{sur}(\mathbb{R}, \mathcal{R})$. De par notre convention dans (4), l'inverse généralisé $F_{\mu}^{-1}$ est continu à droite sur ]0,1[ et il en découle que la seule possibilité pour que $\widetilde{b}$ ne soit éventuellement pas continue à droite en $1 / 2$ est que $\widetilde{b}(u)=+\infty$ pour tout $1 / 2<u<1$. Dans tous les cas, il apparaît à partir du lemme 32 que

$$
\begin{aligned}
B(\mu, \nu) & =\sup _{1 / 2 \leq u<1}(1-u) \widetilde{b}_{\mu, \nu}(u) \\
& =\sup _{1 / 2<u<1}(1-u) \widetilde{b}_{\mu, \nu}(u) \\
& =2 \sup _{0<s<1}(1-s) F_{\mu, \nu}(s)
\end{aligned}
$$

Il resort de cette observation que l'on peut trouver une suite $\left(\epsilon_{n}\right)_{n \in \mathbb{N}}$ de réels strictement positifs convergent vers 0 et une suite $\left(s_{n}\right)_{n \in \mathbb{N}}$ d'éléments de $] 0,1[$ tels que pour tout $n \in \mathbb{N}$,

$$
F_{\mu, \nu} \geq\left(1-\epsilon_{n}\right) \frac{B(\mu, \nu)}{2\left(1-s_{n}\right)} \mathbb{1}_{\left[s_{n}, 1[\right.}
$$

(sauf dans les cas pathologiques où $B(\mu, \nu)=0$, mais l'égalité $A(\mu, \nu)=B(\mu, \nu)$ provient alors trivialement de la seconde inégalité de (2)).

Ainsi d'après les résultats de comparaison de la section 4 , il suffit de montrer que pour tout $s \in] 0,1\left[\right.$ fixé, $\mathcal{A}\left(\mathbb{1}_{[s, 1[}\right)=2(1-s)$ pour obtenir la borne $B(\mu, \nu) \leq A(\mu, \nu)$. Cependant en défaisant la transformation de Girsanov considérée à la fin de la preuve de la proposition 23 , on a que $\mathcal{A}\left(\mathbb{1}_{[s, 1[}\right)$ est l'infimum des constantes $A>0$ telles que

$$
\mathbb{E}_{0}\left[\exp \left(\frac{2}{A} \int_{0}^{1} \mathbb{1}_{[s, 1[}(1-u) B_{u} d B_{u}\right)\right]<+\infty
$$

ou encore, puisque $2 \int_{0}^{1-s} B_{u} d B_{u}=B_{1-s}^{2}-1+s$, l'infimum des constantes $A>0$ telles que

$$
\mathbb{E}_{0}\left[\exp \left(\frac{1}{A} B_{1-s}^{2}\right)\right]<+\infty
$$

d'où l'égalité annoncée $\mathcal{A}\left(\mathbb{1}_{[s, 1[}\right)=2(1-s)$. 
Intéressons-nous ensuite comme annoncé au cas d'égalité $B(\mu, \nu)=A(\mu, \nu)$ quand $\lim \sup _{x \rightarrow M_{\mu}-} b_{\mu, \nu}(x)<B(\mu, \nu)$, et outre les situations où $A(\mu, \nu)=0$ ou $A(\mu, \nu)=+\infty$, montrons que ceci n'est possible dans notre contexte symétrique que s'il existe $0<x \leq M_{\mu}$ et $0<p \leq 1 / 2$ tels que la restriction de $\mu$ à $[-x, x]$ vaut $p\left(\delta_{-x}+\delta_{x}\right)$ et $\int_{x}^{M_{\mu}} 1 / \nu d \lambda=0$ (dans les cas plus réguliers considérés dans l'introduction, cette dernière condition implique que $x=M_{\mu}$ puis que $\mu$ est une distribution de Bernoulli, ainsi que cela était énoncé). Pour nous convaincre de ceci, il suffit de voir que l'égalité $B(\mu, \nu)=A(\mu, \nu) \in \mathbb{R}_{+}^{*}$ sous les conditions précédentes implique que $F_{\mu, \nu}$ est une fonction palier simple, c'est-à-dire qu'il existe $a>0$ et $0<s<1$ tels que $F_{\mu, \nu}=a \mathbb{1}_{[s, 1[}$. Nous allons d'ailleurs procéder par l'absurde : supposons que $F_{\mu, \nu}$ ne soit pas de cette forme, on peut alors trouver $a_{1}, a_{2}>0$ et $0 \leq s_{1}<s_{2}<1$ tels que $F_{\mu, \nu} \geq a_{1} \mathbb{1}_{\left[s_{1}, 1[\right.}+a_{2} \mathbb{1}_{\left[s_{2}, 1[\right.}$, avec $a_{1}\left(1-s_{1}\right)=B(\mu, \nu) / 2$ et $\left(a_{1}+a_{2}\right)\left(1-s_{2}\right) \leq B(\mu, \nu) / 2$ ou $a_{1}\left(1-s_{1}\right) \leq B(\mu, \nu) / 2$ et $\left(a_{1}+a_{2}\right)\left(1-s_{2}\right)=B(\mu, \nu) / 2$. En effet, on voit aisément à partir de la preuve du lemme 32 que s'il existe $\left.x \in] 0, M_{\mu}\right]$ tel que $B(\mu, \nu)=b_{\mu, \nu}(x)$, alors $B(\mu, \nu)=2(1-v) F_{\mu, \nu}(v)$ avec $v=F_{\mu}(x-)$ (on aura aussi noté que si $B(\mu, \nu)$ correspond à un supremum de $b_{\mu, \nu}$ (respectivement de la fonction $s \mapsto 2(1-s) F_{\mu, \nu}(s)$ ) sur un sous-ensemble compact de $] 0, M_{\mu}$ [ (resp. de ]0,1[), alors il est atteint, car l'application $b_{\mu, \nu}$ (resp. $F_{\mu, \nu}$ ) est continue à gauche (resp. à droite) et ne fait que des sauts vers le bas (resp. vers le haut)). En considérant séparément les cas où $B(\mu, \nu)=b_{\mu, \nu}\left(M_{\mu}\right)$ (qui implique en particulier que $\left.\mu\left(\left\{M_{\mu}\right\}\right)>0\right)$ et où $B(\mu, \nu)>b_{\mu, \nu}\left(M_{\mu}\right)$, notre hypothèse implique alors que dans (36) le supremum est atteint sur ]0,1[ (de la même manière on se persuaderait plus généralement que l'utilisation ci-dessus des suites $\left(\epsilon_{n}\right)_{n \in \mathbb{N}}$ et $\left(s_{n}\right)_{n \in \mathbb{N}}$ n'est vraiment justifiée que si $\left.\lim _{n \rightarrow \infty} s_{n}=1\right)$. Un argument de comparaison et le résultat suivant permettent ensuite de voir que

$$
A(\mu, \nu) \geq \mathcal{A}\left(a_{1} \mathbb{1}_{\left[s_{1}, 1[\right.}+a_{2} \mathbb{1}_{\left[s_{2}, 1[\right.}\right)>B(\mu, \nu)
$$

Lemme 37 Soient $a_{1}, a_{2}>0$ et $0<s_{1}<s_{2}<1$, on a

$$
\begin{aligned}
\mathcal{A}\left(a_{1} \mathbb{1}_{\left[s_{1}, 1[\right.}+a_{2} \mathbb{1}_{\left[s_{2}, 1\right]}\right)= & \left(a_{1}+a_{2}\right)\left(1-s_{1}\right)+a_{1}\left(s_{2}-s_{1}\right) \\
& -\sqrt{\left(a_{1}+a_{2}\right)^{2}\left(1-s_{1}\right)^{2}+a_{1}^{2}\left(s_{2}-s_{1}\right)^{2}+2 a_{1}\left(a_{1}-a_{2}\right)\left(s_{2}-s_{1}\right)\left(1-s_{1}\right)} \\
> & 2 \max \left(a_{1}\left(1-s_{1}\right),\left(a_{1}+a_{2}\right)\left(1-s_{2}\right)\right)
\end{aligned}
$$

\section{Preuve :}

Comme au début de cette section, on commence par remarquer que $\mathcal{A}\left(a_{1} \mathbb{1}_{\left[s_{1}, 1[\right.}+a_{2} \mathbb{1}_{\left[s_{2}, 1[\right.}\right)$ n'est autre que l'infimum des constantes $A>0$ telles que

$$
\mathbb{E}_{0}\left[\exp \left(\frac{2}{A}\left\{\left(a_{1}+a_{2}\right) \int_{0}^{1-s_{2}} B_{u} d B_{u}+a_{1} \int_{1-s_{2}}^{1-s_{1}} B_{u} d B_{u}\right\}\right)\right]<+\infty
$$

Or en calculant le terme entre accolades, il apparaît que cette finitude est équivalente à

$$
\mathbb{E}_{0}\left[\exp \left(\frac{a_{1}}{A} B_{1-s_{1}}^{2}+\frac{a_{2}}{A} B_{1-s_{2}}^{2}\right)\right]<+\infty
$$

Cependant en conditionnant par $B_{1-s_{2}}$, on voit que pour $A>2 a_{1}\left(s_{2}-s_{1}\right)$, le membre de gauche vaut aussi

$$
\left(1-2 a_{1}\left(s_{2}-s_{1}\right) / A\right)^{-1 / 2} \mathbb{E}_{0}\left[\exp \left(\left\{\frac{a_{2}}{A}+\frac{a_{1}}{A-2 a_{1}\left(s_{2}-s_{1}\right)}\right\} B_{1-s_{1}}^{2}\right)\right]
$$

ainsi $\mathcal{A}\left(a_{1} \mathbb{1}_{\left[s_{1}, 1[\right.}+a_{2} \mathbb{1}_{\left[s_{2}, 1[\right.}\right)$ est la plus petite solution $A>2 a_{1}\left(s_{2}-s_{1}\right)$ de l'équation du second degré

$$
\frac{a_{2}}{A}+\frac{a_{1}}{A-2 a_{1}\left(s_{2}-s_{1}\right)}=\frac{1}{2\left(1-s_{2}\right)}
$$


d'où les résultats annoncés (la majoration stricte se lisant d'ailleurs plus facilement sur l'égalité ci-dessus que sur l'expression de $\left.\mathcal{A}\left(a_{1} \mathbb{1}_{\left[s_{1}, 1[\right.}+a_{2} \mathbb{1}_{\left[s_{2}, 1[\right.}\right)\right)$.

Après avoir utilisé des fonctions à un palier, puis deux, nous allons ci-dessous considérer une infinité de paliers pour exhiber une application $H:] 0,1[\rightarrow \mathbb{R}$ telle que

$$
\limsup _{s \rightarrow 1-}(1-s) H(s)=1 \quad \text { et } \quad \lim _{\eta \rightarrow 0_{+}} \mathcal{A}\left(H_{\eta}\right)=2
$$

où l'on a posé pour tout $0<\eta<1$,

$$
\forall s \in] 0,1\left[, \quad H_{\eta}(s):=H((1-\eta) \wedge s)\right.
$$

C'est ensuite une retranscription désormais usuelle que d'en déduire un couple $(\mu, \nu)$ formé d'une probabilité et d'une mesure symétriques tel que $M_{\mu}=+\infty$,

$$
\limsup _{x \rightarrow+\infty} b_{\mu, \nu}(x)=B(\mu, \nu) \quad \text { et } \quad A(\mu, \nu)=B(\mu, \nu)
$$

(on peut choisir par exemple $\nu=\lambda$ et $\mu$ sera alors une somme pondérée de masse de Dirac divergentes symétriquement vers l'infini). Pour reprendre une terminologie heuristique de l'introduction, il s'agit aussi d'une situation où $A(\mu, \nu)$ mesure la difficulté de revenir de l'infini, car il est possible de perturber localement $\nu$ sans modifier la valeur de $A(\mu, \nu)$, mais cette difficulté provient d'une suite de points retardants divergente vers l'infini plus que d'une "difficulté presque homogène" comme dans la proposition 2.

Un peu plus rigoureusement maintenant, nous cherchons donc une fonction $H$ satisfaisant (37) de la forme suivante

$$
\forall s \in] 0,1\left[, \quad H(s):=\sum_{n \in \mathbb{N}} a_{n} \mathbb{1}_{\left[s_{n}, 1[\right.}(s)\right.
$$

avec $\left(s_{n}\right)_{n \in \mathbb{N}}$ une suite strictement croissante d'éléments de ]0,1[ convergente vers 1 et $\left(a_{n}\right)_{n \in \mathbb{N}}$ une suite de réels strictement positifs.

Pour assurer la première condition de (37), nous allons imposer que

$$
\lim _{n \rightarrow \infty}\left(1-s_{n}\right) H\left(s_{n}\right)=1 \quad \text { et } \quad \forall n \in \mathbb{N}, \quad\left(1-s_{n}\right) H\left(s_{n}\right)<1
$$

Ceci implique que $\sup _{0<s<1}(1-s) H(s)=1$ et donc d'après le début de cette section,

$$
\lim _{\eta \rightarrow 0_{+}} \mathcal{A}\left(H_{\eta}\right) \geq 2
$$

(rappelons que cette limite existe par monotonicité de $\mathcal{A}\left(H_{\eta}\right)$ en $\left.\eta \in\right] 0,1[$ ). Pour obtenir la deuxième condition de (37), il suffit donc de montrer que

$$
\forall N \in \mathbb{N}, \quad \mathcal{A}\left(S_{N}\right) \leq 2
$$

avec $S_{N}:=H_{1-s_{N}}=\sum_{0 \leq n \leq N} a_{n} \mathbb{1}_{\left[s_{n}, 1[\right.}$. Pour disposer d'un critère allant dans ce sens, définissons une nouvelle suite $\left(\widetilde{a}_{n}\right)_{n \in \mathbb{N}}$ (à valeurs dans $\overline{\mathbb{R}}_{+}$) de la manière récursive suivante : $\widetilde{a}_{0}=a_{0}$ puis pour tout $n \in \mathbb{N}$,

$$
\widetilde{a}_{n+1}:=a_{n+1}+\frac{\widetilde{a}_{n}}{\left(1-\left(s_{n+1}-s_{n}\right) \widetilde{a}_{n}\right)_{+}}
$$

(ainsi s'il existe $n \in \mathbb{N}$ tel que $\left(s_{n+1}-s_{n}\right) \widetilde{a}_{n} \geq 1$, on aura pour tout $m>n, \widetilde{a}_{m}=+\infty$ ). On a alors 
Lemme 38 Pour tout $N \in \mathbb{N}$, on a équivalence entre

$$
\mathbb{E}_{0}\left[\exp \left(\frac{1}{2} \sum_{0 \leq n \leq N} a_{n} B_{1-s_{n}}^{2}\right)\right]<+\infty
$$

et

$$
\left(1-s_{N}\right) \widetilde{a}_{N}<1
$$

\section{Preuve :}

Pour évaluer l'espérance ci-dessus, on commence par conditionner par la trajectoire du mouvement brownien jusqu'au temps $1-s_{1}$, ce qui nous amène par la propriété de Markov à nous intéresser à

$$
\mathbb{E}_{0}\left[\exp \left(\frac{1}{2} a_{0} B_{1-s_{0}}^{2}\right) \mid B_{1-s_{1}}\right]=\left(\left(1-a_{0}\left(s_{1}-s_{0}\right)\right)_{+}\right)^{-\frac{1}{2}} \exp \left(\frac{a_{1}}{2\left(1-a_{0}\left(s_{1}-s_{0}\right)\right)_{+}} B_{1-s_{1}}^{2}\right)
$$

expression qui vaut $+\infty$ si $a_{0}\left(s_{1}-s_{0}\right) \geq 1$. Sinon, on obtient de la sorte que

$$
\begin{aligned}
& \mathbb{E}_{0}\left[\exp \left(\frac{1}{2} \sum_{0 \leq n \leq N} a_{n} B_{1-s_{n}}^{2}\right)\right] \\
& =\left(\left(1-a_{0}\left(s_{1}-s_{0}\right)\right)_{+}\right)^{-\frac{1}{2}} \mathbb{E}_{0}\left[\exp \left(\frac{1}{2} \widetilde{a}_{1} B_{1-s_{1}}^{2}+\frac{1}{2} \sum_{2 \leq n \leq N} a_{n} B_{1-s_{n}}^{2}\right)\right]
\end{aligned}
$$

On poursuit par récurrence pour obtenir que si pour tout $0 \leq n<N$ on a $\widetilde{a}_{n}\left(s_{n+1}-s_{n}\right) \geq 1$, alors

$$
\mathbb{E}_{0}\left[\exp \left(\frac{1}{2} \sum_{0 \leq n \leq N} a_{n} B_{1-s_{n}}^{2}\right)\right]=\left(\prod_{0 \leq n<N}\left(1-\widetilde{a}_{n}\left(s_{n+1}-s_{n}\right)\right)\right)^{-\frac{1}{2}} \mathbb{E}_{0}\left[\exp \left(\frac{\widetilde{a}_{N}}{2} B_{1-s_{N}}^{2}\right)\right]
$$

d'où en fin de compte le résultat annoncé.

Cependant, par des calculs similaires à ceux déjà effectués dans cette section, on reconnaît que pour tout $N \in \mathbb{N}, \mathcal{A}\left(S_{N}\right)$ est l'infimum des $A>0$ tels que

$$
\mathbb{E}_{0}\left[\exp \left(\frac{1}{2} \sum_{0 \leq n \leq N} a_{n} B_{1-s_{n}}^{2}\right)\right]<+\infty
$$

Ainsi pour assurer (39), il suffit d'avoir

$$
\forall N \in \mathbb{N}, \quad\left(1-s_{N}\right) \widetilde{a}_{N}<1
$$

et nous allons choisir des suites $\left(a_{n}\right)_{n \in \mathbb{N}}$ et $\left(s_{n}\right)_{n \in \mathbb{N}}$ comme ci-dessus qui vérifient cette propriété. Pour cela, donnons-nous a priori une suite $\left(\epsilon_{n}\right)_{n \in \mathbb{N}}$ de réels strictement positifs décroissante vers 0 et imposons que

$$
\forall n \in \mathbb{N}, \quad\left(1-s_{n}\right) a_{n}=1-\epsilon_{n}
$$

ainsi nous n'avons à nous occuper que de la suite $\left(s_{n}\right)_{n \in \mathbb{N}}$. On procède par récurrence : on commence par prendre $s_{0}=1 / 2$; on a bien $\left(1-s_{0}\right) \widetilde{a}_{0}=\left(1-s_{0}\right) a_{0}=1-\epsilon_{0}<1$. Supposons ensuite $s_{0}, \ldots, s_{n}$ construits et montrons que l'on peut choisir $s_{n}<s_{n+1}<1$ tel que

$$
\begin{aligned}
1-\epsilon_{n+1}<\left(1-s_{n+1}\right) \widetilde{a}_{n+1} & <1 \\
1-\epsilon_{n+1}<\left(1-s_{n+1}\right)\left(a_{0}+\cdots+a_{n+1}\right) & <1
\end{aligned}
$$


ce qui terminera de nous convaincre des comportements voulus.

Pour le premier encadrement, on note que

$$
\left(1-s_{n+1}\right) \widetilde{a}_{n+1}=1-\epsilon_{n+1}+\frac{\left(1-s_{n+1}\right) \widetilde{a}_{n}}{1-\left(s_{n+1}-s_{n}\right) \widetilde{a}_{n}}
$$

d'où (40) si l'on choisit $s_{n+1}$ assez proche de 1 . De la même manière, on peut prendre $s_{n+1}$ éventuellement encore plus proche de 1 pour satisfaire la seconde borne (41). D'où le contre-exemple escompté.

On peut s'amuser à effectuer d'autres calculs explicites pour le type précédent (38) de fonction $H$ à infinité de paliers. Par exemple en choisissant $0<\rho<1$ puis en posant pour tout $n \in \mathbb{N}$,

$$
s_{n}:=1-\rho^{1+n} \quad \text { et } \quad a_{n}:=\left(\rho^{-1}-1\right) \rho^{-n}
$$

on obtient que

$$
\sup _{0<s<1}(1-s) H(s)=1 \quad \text { et } \quad \lim _{\eta \rightarrow 0_{+}} \mathcal{A}\left(H_{\eta}\right)=2(1+\sqrt{\rho})^{2}
$$

c'est-à-dire que si $\left(\mu_{\rho}, \nu_{\rho}\right)$ est un couple correspondant formé d'une probabilité et d'une mesure symétrique, alors le quotient $B\left(\mu_{\rho}, \nu_{\rho}\right) / A\left(\mu_{\rho}, \nu_{\rho}\right)$ décrit tout l'intervalle ]1,4[ quand $\eta$ parcourt ] $0,1[$.

\section{$7 \quad$ Un équivalent discret}

L'apparition dans la constante $B(\mu, \nu)$, pour une probabilité $\mu$ et une mesure $\nu$ symétriques données, d'une densité de Radon-Nikodym de $\nu$ par rapport à $\lambda$ est directement liée au fait que l'on considère une dérivée "usuelle" (à réinterpréter comme une dérivation par rapport à la mesure de Lebesgue) dans l'inégalité de Poincaré (1). Et si l'on désire restreindre le rôle de la mesure de Lebesgue dans ce type de problèmes, on peut s'intéresser plutôt à la quantité $\bar{A}(\mu, \nu)$ définie comme l'infimum des $A>0$ tels que

$$
\frac{1}{4} \int_{x<y}\left(\int_{[x, y[} f(u) \nu(d u)\right)^{2} \mu(d x) \mu(d y) \leq A \nu\left[f^{2}\right]
$$

La généralité et la commodité d'une telle formulation ont été mis en évidence et exploitées par Sinnamon [41] pour les inégalités de Hardy, qu'il a étudié dans ce contexte. Cependant notre but dans cette dernière section n'est pas de tenter d'adapter les considérations précédentes à ce cadre, nous nous contenterons de retrouver l'inégalité (2) et la proposition 2 dans le cas où $\nu$ est une somme pondérée symétriquement de masses de Dirac sur $\mathbb{Z}$, car il s'agit d'une situation particulièrement intéressante lors de l'étude des chaînes de Markov, correspondant aux processus de vie et de mort (voir par exemple le livre [6] de Chen ou [27] pour des estimées de trou spectral à partir d'inégalités de Hardy, qui d'ailleurs auraient pu s'obtenir à partir de l'article de Sinnamon [41]).

Dans ce contexte, on préfère voir $\nu$ comme une mesure sur l'ensemble $E:=\{\{n, n+1\}, n \in \mathbb{Z}\}$ des arêtes naturelles de $\mathbb{Z}$. On dispose donc d'une probabilité $\mu$ sur $\mathbb{Z}$ et d'une mesure $\nu$ sur $E$, toutes deux symétriques, et on cherche à évaluer $\widetilde{A}(\mu, \nu)$ l'infimum des $A>0$ tels que pour toute fonction $f: \mathbb{Z} \rightarrow \mathbb{R}$ appartenant à $\mathbb{L}^{2}(\mu)$,

$$
\operatorname{Var}(f, \mu) \leq A \sum_{e \in E} \nu(e)(d f(e))^{2}
$$

où pour toute arête $e=\{n, n+1\} \in E$, on a posé $d f(e):=f(n+1)-f(n)$.

Introduisons

$$
\widetilde{B}(\mu, \nu):=\sup _{N \in \mathbb{N}} \sum_{E \ni e \subset[0, N]} \frac{1}{\nu(e)} \mu([N,+\infty[)
$$


et on dira que la condition $(\mathrm{C})$ est réalisée si

$$
\widetilde{B}(\mu, \nu)=\lim _{N \rightarrow \infty} \sum_{E \ni e \subset[0, N]} \frac{1}{\nu(e)} \mu([N+1,+\infty[)
$$

(on aura noté le petit décalage par rapport à la définition précédente, ceci revient aussi à demander que $\widetilde{B}(\mu, \nu)=\lim _{N \rightarrow \infty} \sum_{E \ni e \subset[0, N]} \frac{1}{\nu(e)} \mu\left(\left[N,+\infty[)\right.\right.$ et $\left.\lim _{N \rightarrow \infty} \sum_{E \ni e \subset[0, N]} \frac{1}{\nu(e)} \mu(\{N\})=0\right)$.

Notre objectif ici est simplement d'obtenir comme conséquence des résultats précédents la

Proposition 39 Sous les hypothèses ci-dessus, on a

$$
\widetilde{B}(\mu, \nu) \leq \widetilde{A}(\mu, \nu) \leq 4 \widetilde{B}(\mu, \nu)
$$

et sous la condition $(C)$,

$$
\widetilde{A}(\mu, \nu)=4 \widetilde{B}(\mu, \nu)
$$

L'argument sous-jacent est très élémentaire et consiste à réinterpréter $\widetilde{A}(\mu, \nu)$ comme la constante de Poincaré $A(\mu, \widetilde{\nu})$ avec la mesure

$$
\widetilde{\nu}:=\sum_{e \in E} \nu(e) \lambda_{e}
$$

où $\lambda_{e}$ désigne la restriction de $\lambda$ à $] n, n+1[$ si $e=\{n, n+1\} \in E$.

En effet, soit $\mathcal{D}_{+}(\mathbb{R})$ le sous-ensemble de $\mathcal{B}_{+}(\mathbb{R})$ des fonctions boréliennes positives sur $(\mathbb{R}, \mathcal{R})$, qui sont constantes sur chacun des intervalles $[n, n+1[$, pour $n \in \mathbb{Z}$. On a clairement

$$
\begin{aligned}
A(\mu, \widetilde{\nu}) & =\sup _{f \in \mathcal{B}_{+}(\mathbb{R})} \frac{1}{2} \frac{\int\left(\int_{x}^{y} f(u) d u\right)^{2} \mu(d x) \mu(d y)}{\widetilde{\nu}\left[f^{2}\right]} \\
& \geq \sup _{f \in \mathcal{D}_{+}(\mathbb{R})} \frac{1}{2} \frac{\int\left(\int_{x}^{y} f(u) d u\right)^{2} \mu(d x) \mu(d y)}{\widetilde{\nu}\left[f^{2}\right]} \\
& =\widetilde{A}(\mu, \nu)
\end{aligned}
$$

Réciproquement, soit $f \in \mathcal{B}_{+}(\mathbb{R})$ donné et associons lui la fonction $\tilde{f} \in \mathcal{D}_{+}(\mathbb{R})$ définie par

$$
\forall x \in \mathbb{R}, \quad \widetilde{f}(x):=\int_{\lfloor x\rfloor}^{\lfloor x\rfloor+1} f(u) d u
$$

Du fait que $\mu$ est portée par $\mathbb{Z}$, on a évidemment

$$
\int\left(\int_{x}^{y} f(u) d u\right)^{2} \mu(d x) \mu(d y)=\int\left(\int_{x}^{y} \widetilde{f}(u) d u\right)^{2} \mu(d x) \mu(d y)
$$

D'autre part, l'inégalité de Cauchy-Schwarz et la forme particulière de $\widetilde{\nu}$ assurent que

$$
\begin{aligned}
\forall n \in \mathbb{Z}, \quad \int_{n}^{n+1} f^{2} d \widetilde{\nu} & \geq\left(\int_{n}^{n+1} f d \lambda\right)^{2} \widetilde{\nu}(] n, n+1[) \\
& =\int_{n}^{n+1} \widetilde{f}^{2} d \widetilde{\nu}
\end{aligned}
$$

Ainsi on a

$$
\frac{\int\left(\int_{x}^{y} f(u) d u\right)^{2} \mu(d x) \mu(d y)}{\widetilde{\nu}\left[f^{2}\right]} \leq \frac{\int\left(\int_{x}^{y} \widetilde{f}(u) d u\right)^{2} \mu(d x) \mu(d y)}{\widetilde{\nu}\left[\widetilde{f}^{2}\right]}
$$


ce qui montre que l'inégalité (42) est en fait une égalité, d'où en fin de compte $\widetilde{A}(\mu, \nu)=A(\mu, \widetilde{\nu})$. Par ailleurs, on note que la fonction $b_{\mu, \widetilde{\nu}}$ est croissante sur chacun des intervalles $\left.] n, n+1\right]$, pour $n \in \mathbb{N}$, et fait (sauf si $\mu(n)=0$ ) un saut vers le bas en $n_{+}$. Il en découle que

$$
\begin{aligned}
B(\mu, \widetilde{\nu}) & =\sup _{n \in \mathbb{N}} b_{\mu, \widetilde{\nu}}(n) \\
& =\widetilde{B}(\mu, \nu)
\end{aligned}
$$

Les premières bornes de la proposition 39 découlent alors immédiatement de (2) et le cas d'égalité sous (C) provient de la proposition 2, car on aura remarqué (le cas trivial où $\nu$ est nulle étant écarté) que cette condition est exactement celle qui assure d'une part que $M_{\mu}=+\infty$ (seule manière d'avoir $\mu\left(\left\{M_{\mu}\right\}\right)=0$ dans ce cadre discret) et d'autre part que $\lim _{x \rightarrow+\infty} b_{\mu, \widetilde{\nu}}(x)=B(\mu, \widetilde{\nu})$.

Remarque 40 Nous venons de ramener le cas discret au cas continu, mais signalons que l'opération inverse est également possible. Aux réductions de la section 2 près, soient $\mu$ et $\nu$ une probabilité et une mesure symétriques sur ] - 1, 1[ et équivalentes à la restriction de $\lambda$ sur cet ensemble (disons, avec des densités régulières minorées et majorées par des réels strictement positifs).

Introduisons une famille $\left(x_{n, k}\right)_{n \in \mathbb{N}, k \in \mathbb{Z}}$ d'éléments sur ] - 1, 1 [ par une récurrence sur l'indice $n \in \mathbb{N}$. On commence par définir pour tout $k \in \mathbb{Z}$,

$$
x_{0, k}:=\operatorname{sign}(k)\left(1-2^{-|k|}\right)
$$

Puis si pour $n \in \mathbb{N}$, la suite $\left(x_{n, k}\right)_{k \in \mathbb{Z}}$ a été construite, on lui rajoute tous les points milieux $\left(x_{n, k}+x_{n, k+1}\right) / 2$, pour $k \in \mathbb{Z}$, pour obtenir une nouvelle suite croissante $\left(x_{n+1, k}\right)_{k \in \mathbb{Z}}$ telle que $x_{n+1,0}=0$.

Pour $n \in \mathbb{N}$, soit $\mu_{n,+}$ (respectivement $\mu_{n,-}$ ) la probabilité symétrique donnée par

$$
\left.\left.\mu_{n,+}\left(\left\{x_{n, 0}\right\}\right):=\mu(\{0\}) \quad \text { et } \quad \forall k \in \mathbb{N}^{*}, \quad \mu_{n,+}\left(\left\{x_{n, k}\right\}\right):=\mu(] x_{n, k-1}, x_{n, k}\right]\right)
$$

(resp. $\mu_{n,-}\left(\left\{x_{n, 0}\right\}\right):=\mu(] x_{n,-1}, x_{n, 1}[)$ et $\mu_{n,-}\left(\left\{x_{n, k}\right\}\right):=\mu\left(\left[x_{n, k}, x_{n, k+1}[)\right.\right.$ pour $\left.k \in \mathbb{N}^{*}\right)$. On notera aussi $\widetilde{\mu}_{n,+}$ (resp. $\widetilde{\mu}_{n,-}$ ) la probabilité symétrique sur $\mathbb{Z}$ définie par

$$
\forall k \in \mathbb{Z}, \quad \widetilde{\mu}_{n,+}(k):=\mu_{n,+}\left(\left\{x_{n, k}\right\}\right)
$$

(resp. $\left.\widetilde{\mu}_{n,-}(k):=\mu_{n,-}\left(\left\{x_{n, k}\right\}\right)\right)$. Considérons ensuite la mesure $\widetilde{\nu}_{n}$ symétrique sur les arêtes de $E$ donnée par

$$
\forall\{k, k+1\} \in E, \quad \nu_{n}(\{k, k+1\}):=\frac{1}{\int_{x_{n, k}}^{x_{n, k+1}} \frac{1}{\nu} d \lambda}
$$

Vérifions que $A\left(\mu_{n,+}, \nu\right)=\widetilde{A}\left(\widetilde{\mu}_{n,+}, \widetilde{\nu}_{n}\right)$ pour tout $n \in \mathbb{N}$ (de manière similaire on aura $A\left(\mu_{n,-}, \nu\right)=$ $\left.\widetilde{A}\left(\widetilde{\mu}_{n,-}, \widetilde{\nu}_{n}\right)\right)$. Soit $f \in \mathcal{B}_{+}(\mathbb{R})$, la variance $\operatorname{Var}\left(f, \mu_{n,+}\right)$ ne dépend que des valeurs de $f$ sur la suite $\left(x_{n, k}\right)_{k \in \mathbb{Z}}$. Or pour $k \in \mathbb{Z}$, on sait minimiser la quantité $\int_{x_{n, k}}^{x_{n, k+1}}\left(g^{\prime}\right)^{2} d \nu$ sur l'ensemble des fonctions $g$ absolument continues sur ] - 1,1[ satisfaisant la contrainte $g\left(x_{n, l}\right)=f\left(x_{n, l}\right)$ pour tout $l \in \mathbb{Z}$ (ou seulement pour $l=k, k+1$ ). Il s'agit en effet d'une application immédiate de l'inégalité de Cauchy-Schwarz et on obtient $\left(f\left(x_{n, k+1}\right)-f\left(x_{n, k}\right)\right)^{2} \widetilde{\nu}_{n}(\{k, k+1\})$ pour valeur minimale. Il en découle que

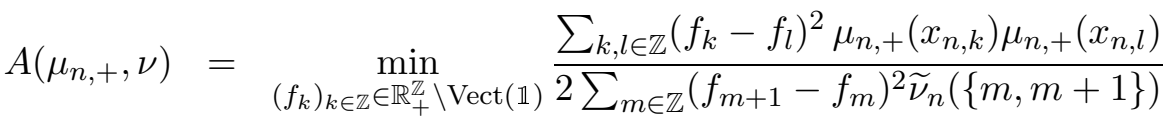

$$
\begin{aligned}
& =\widetilde{A}\left(\widetilde{\mu}_{n,+}, \widetilde{\nu}_{n}\right)
\end{aligned}
$$

Dans le même registre, la forme particulière de $\widetilde{\nu}_{n}$ permet de voir que

$$
\begin{aligned}
& B\left(\mu_{n,+}, \nu\right)=\widetilde{B}\left(\widetilde{\mu}_{n,+}, \widetilde{\nu}_{n}\right) \\
& B\left(\mu_{n,-}, \nu\right)=\widetilde{B}\left(\widetilde{\mu}_{n,-}, \widetilde{\nu}_{n}\right)
\end{aligned}
$$


Ainsi si l'on disposait a priori de renseignements sur les inégalités de Poincaré discrètes (comme la proposition 39 et des résultats de comparaison), on pourrait ensuite les étendre au cas continu. On obtiendrait notamment que la suite $\left(A\left(\mu_{n,+}, \nu\right)\right)_{n \in \mathbb{N}}$ (respectivement $\left.\left(A\left(\mu_{n,-}, \nu\right)\right)_{n \in \mathbb{N}}\right)$ est décroissante (resp. croissante) et converge vers $A(\mu, \nu)$ (on retrouverait en particulier pour tout $n \in \mathbb{N}$, les bornes $A\left(\mu_{n,-}, \nu\right) \leq A(\mu, \nu) \leq A\left(\mu_{n,+}, \nu\right)$, qui pour nous s'obtiennent directement à partir des résultats de comparaison de la section 4$)$.

Remerciements : je tiens à exprimer toute ma gratitude envers Catherine DonatiMartin, Michel Ledoux et Marc Yor pour leurs conseils lors de la recherche bibliographique sur le sujet. Evidemment cette reconnaissance ne me dédouane en rien des lacunes dont sont certainement encore entachées les références suivantes! Par ailleurs, je remercie aussi le Laboratoire de Statistique et Probabilités (UMR 5583, Université Paul Sabatier et CNRS), où une partie de ce travail a été effectué.

\section{Références}

[1] F. Barthe, P. Cattiaux, and C. Roberto. Interpolated inequalities between exponential and Gaussian, Orlicz hypercontractivity and isoperimetry. Préprint, 2004.

[2] F. Barthe and C. Roberto. Sobolev inequalities for probability measures on the real line. Studia Math., 159(3) :481-497, 2003. Dedicated to Professor Aleksander Pełczyński on the occasion of his 70th birthday (Polish).

[3] S. G. Bobkov and F. Götze. Exponential integrability and transportation cost related to logarithmic Sobolev inequalities. J. Funct. Anal., 163(1) :1-28, 1999.

[4] S.G. Bobkov and F. Götze. Muckenhoupt's condition via Riccati and Sturm-Liouville equations. Préprint, 2002.

[5] Djalil Chafaï. Entropies, convexity, and functional inequalities. J. Math. Kyoto Univ., 44(2) :325-363, 2004.

[6] Mu-Fa Chen. From Markov chains to non-equilibrium particle systems. World Scientific Publishing Co. Inc., River Edge, NJ, second edition, 2004.

[7] K. L. Chung and K. M. Rao. Feynman-Kac functional and the Schrödinger equation. In Seminar on Stochastic Processes, 1981 (Evanston, Ill., 1981), volume 1 of Progr. Prob. Statist., pages 1-29. Birkhäuser Boston, Mass., 1981.

[8] K. L. Chung and R. J. Williams. Introduction to stochastic integration. Probability and its Applications. Birkhäuser Boston Inc., Boston, MA, second edition, 1990.

[9] Kai Lai Chung and Zhong Xin Zhao. From Brownian motion to Schrödinger's equation, volume 312 of Grundlehren der Mathematischen Wissenschaften [Fundamental Principles of Mathematical Sciences]. Springer-Verlag, Berlin, 1995.

[10] Yves Colin de Verdière. Spectres de graphes, volume 4 of Cours Spécialisés [Specialized Courses]. Société Mathématique de France, Paris, 1998.

[11] R. Courant and D. Hilbert. Methods of mathematical physics. Vol. I. Interscience Publishers, Inc., New York, N.Y., 1953.

[12] Persi Diaconis and Daniel Stroock. Geometric bounds for eigenvalues of Markov chains. Ann. Appl. Probab., 1(1) :36-61, 1991.

[13] C. Donati-Martin and M. Yor. Mouvement brownien et inégalité de Hardy dans $L^{2}$. In Séminaire de Probabilités, XXIII, volume 1372 of Lecture Notes in Math., pages 315-323. Springer, Berlin, 1989. 
[14] Nelson Dunford and Jacob T. Schwartz. Linear operators. Part II. Wiley Classics Library. John Wiley \& Sons Inc., New York, 1988. Spectral theory. Selfadjoint operators in Hilbert space, With the assistance of William G. Bade and Robert G. Bartle, Reprint of the 1963 original, A Wiley-Interscience Publication.

[15] Masatoshi Fukushima, Yōichi Ōshima, and Masayoshi Takeda. Dirichlet forms and symmetric Markov processes, volume 19 of de Gruyter Studies in Mathematics. Walter de Gruyter \& Co., Berlin, 1994.

[16] Ivan Gentil, Arnaud Guillin, and Laurent Miclo. Modified logarithmic Sobolev inequalities and transportation inequalities. Préprint, 2004.

[17] James Glimm and Arthur Jaffe. Quantum physics. Springer-Verlag, New York, second edition, 1987. A functional integral point of view.

[18] G. H. Hardy, J. E. Littlewood, and G. Pólya. Inequalities. Cambridge Mathematical Library. Cambridge University Press, Cambridge, 1988. Reprint of the 1952 edition.

[19] Philip Hartman. Ordinary differential equations. Birkhäuser Boston, Mass., second edition, 1982.

[20] Richard Holley and Daniel Stroock. Simulated annealing via Sobolev inequalities. Comm. Math. Phys., 115(4) :553-569, 1988.

[21] Richard A. Holley, Shigeo Kusuoka, and Daniel W. Stroock. Asymptotics of the spectral gap with applications to the theory of simulated annealing. J. Funct. Anal., 83(2) :333-347, 1989.

[22] Nabil Kahale. A semidefinite bound for mixing rates of Markov chains. In Proceedings of the Workshop on Randomized Algorithms and Computation (Berkeley, CA, 1995), volume 11, pages 299-313, 1997.

[23] R. Mansuy. On a one-parameter generalization of the Brownian bridge and associated quadratic functionals. J. Theoret. Probab., 17(4) :1021-1029, 2004.

[24] Moshe Marcus, Victor J. Mizel, and Yehuda Pinchover. On the best constant for Hardy's inequality in $\mathbf{R}^{n}$. Trans. Amer. Math. Soc., 350(8) :3237-3255, 1998.

[25] Tanya Matskewich and Pavel E. Sobolevskii. The best possible constant in generalized Hardy's inequality for convex domain in $\mathbf{R}^{n}$. Nonlinear Anal., 28(9) :1601-1610, 1997.

[26] Vladimir G. Maz'ja. Sobolev spaces. Springer Series in Soviet Mathematics. Springer-Verlag, Berlin, 1985. Translated from the Russian by T. O. Shaposhnikova.

[27] L. Miclo. An example of application of discrete Hardy's inequalities. Markov Process. Related Fields, 5(3) :319-330, 1999.

[28] Laurent Miclo. Sur l'inégalité de Sobolev logarithmique des opérateurs de Laguerre à petit paramètre. In Séminaire de Probabilités, XXXVI, volume 1801 of Lecture Notes in Math., pages 222-229. Springer, Berlin, 2003.

[29] Benjamin Muckenhoupt. Hardy's inequality with weights. Studia Math., 44 :31-38, 1972. Collection of articles honoring the completion by Antoni Zygmund of 50 years of scientific activity, I.

[30] Jacques Neveu. Martingales à temps discret. Masson et Cie, éditeurs, Paris, 1972.

[31] G. Peccati and M. Yor. Hardy's inequality in $L^{2}([0,1])$ and principal values of Brownian local times. Préprint, 2001.

[32] G. Peccati and M. Yor. Four limit theorems for quadratic functionals of Brownian motion and Brownian bridge. Préprint à paraître dans Fields Institute Communications, 2004.

[33] Ross Pinsky. A spectral criterion for the finiteness or infiniteness of stopped Feynman-Kac functionals of diffusion processes. Ann. Probab., 14(4) :1180-1187, 1986.

[34] Ross G. Pinsky. Positive harmonic functions and diffusion, volume 45 of Cambridge Studies in Advanced Mathematics. Cambridge University Press, Cambridge, 1995. 
[35] Jim Pitman and Marc Yor. A decomposition of Bessel bridges. Z. Wahrsch. Verw. Gebiete, 59(4) :425-457, 1982.

[36] Jim Pitman and Marc Yor. Sur une décomposition des ponts de Bessel. In Functional analysis in Markov processes (Katata/Kyoto, 1981), volume 923 of Lecture Notes in Math., pages 276285. Springer, Berlin, 1982.

[37] Michael Reed and Barry Simon. Methods of modern mathematical physics. IV. Analysis of operators. Academic Press [Harcourt Brace Jovanovich Publishers], New York, 1978.

[38] Daniel Revuz and Marc Yor. Continuous martingales and Brownian motion, volume 293 of Grundlehren der Mathematischen Wissenschaften [Fundamental Principles of Mathematical Sciences]. Springer-Verlag, Berlin, third edition, 1999.

[39] Walter Rudin. Real and complex analysis. McGraw-Hill Book Co., New York, third edition, 1987.

[40] Laurent Saloff-Coste. Lectures on finite Markov chains. In Lectures on probability theory and statistics (Saint-Flour, 1996), volume 1665 of Lecture Notes in Math., pages 301-413. Springer, Berlin, 1997.

[41] Gord Sinnamon. Four questions related to Hardy's inequality. In Function spaces and applications (Delhi, 1997), pages 255-266. Narosa, New Delhi, 2000.

[42] Marc Yor. Some aspects of Brownian motion. Part I. Lectures in Mathematics ETH Zürich. Birkhäuser Verlag, Basel, 1992. Some special functionals.

Laurent.Miclo@latp.univ-mrs.fr

Laboratoire d'Analyse, Topologie, Probabilités, UMR 6632

Centre de Mathématiques et Informatique

Université de Provence et Centre National de la Recherche Scientifique

39, rue F. Joliot-Curie

13453 Marseille cedex 13, France 